\title{
ATR 2A/2B As-Run Neutronics Uncertainty Analysis
}

Hiroshi Sagara

Gray S. Chang

October 2009

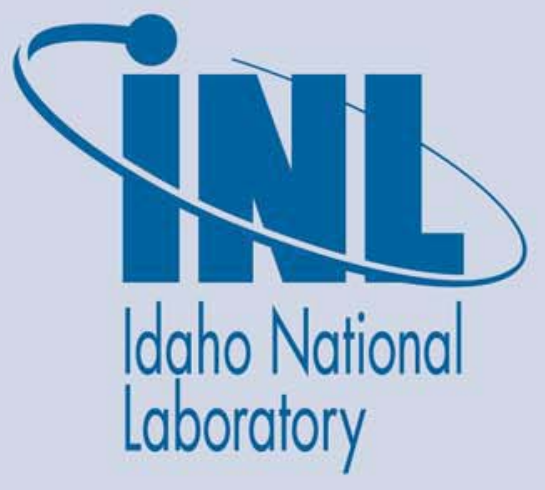

The INL is a U.S. Department of Energy National Laboratory operated by Battelle Energy Alliance 
INL/EXT-09-17197

\section{ATR 2A/2B As-Run Neutronics Uncertainty Analysis}

Hiroshi Sagara

Gray S. Chang

October 2009

\section{Idaho National Laboratory \\ Idaho Falls, Idaho 83415}

http://www.inl.gov

Prepared for the

U.S. Department of Energy

Office of Nuclear Energy

Under DOE Idaho Operations Office

Contract DE-AC07-05ID14517 


\section{ATR 2A/2B As-Run}

Neutronics Uncertainty

Analysis

Advanced Fuel Cycle Initiative

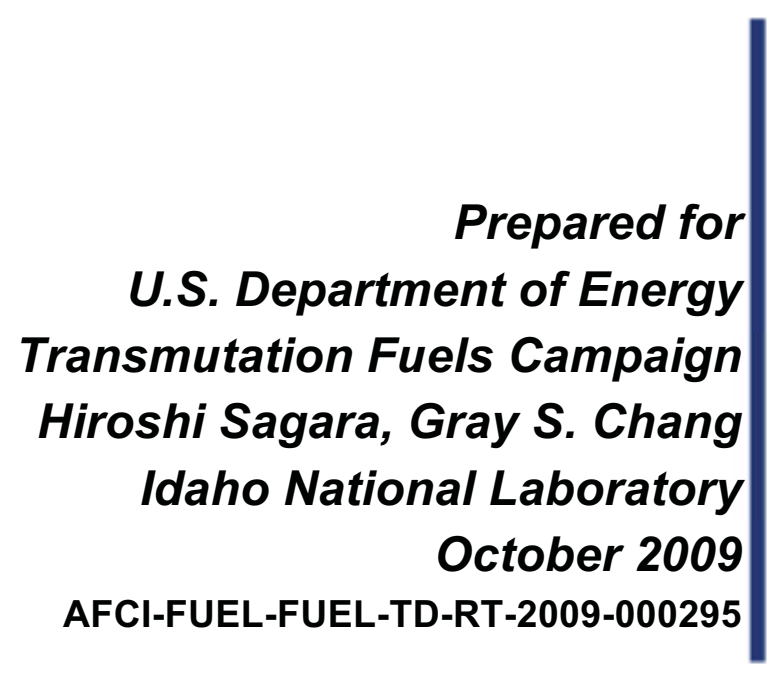

.

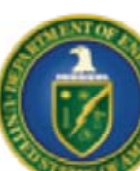




\section{DISCLAIMER}

This information was prepared as an account of work sponsored by an agency of the U.S. Government. Neither the U.S. Government nor any agency thereof, nor any of their employees, makes any warranty, expressed or implied, or assumes any legal liability or responsibility for the accuracy, completeness, or usefulness, of any information, apparatus, product, or process disclosed, or represents that its use would not infringe privately owned rights. References herein to any specific commercial product, process, or service by trade name, trade mark, manufacturer, or otherwise, does not necessarily constitute or imply its endorsement, recommendation, or favoring by the U.S. Government or any agency thereof. The views and opinions of authors expressed herein do not necessarily state or reflect those of the U.S. Government or any agency thereof. 


\section{ABSTRACT}

Neutronics calculations contain several uncertainties such as nuclear data, Monte-Carlo (MC) statistics deviation of the neutron flux tally, initial mass load, power history, etc. Minor Actinides (MAs) have shortage of measured nuclear data (cross-section, thermal property, etc.) and have large uncertainty based on nuclear data. We need to consider the uncertainty caused by nuclear data, especially to treat MA loaded fuel in future reactor designs, safety analysis, and irradiation testing of fuel for these reactors.

Uncertainty analysis relating to calculations for AFC-2A and AFC-2B has been performed. The methodology to treat the nuclear cross section covariance and global uncertainty in the depletion and buildup calculation were established by additions to the current MCWO code system with interface to the ORIGEN Library. This uncertainty analysis methodology was applied to AFC-2A and AFC-2B irradiation analysis, and uncertainties of HM density, elemental composition, isotope composition and burnup are calculated. As a result, the sensitivity of cross section errors are evaluated coupled with original as-run calculations by MCWO, and the importance of cross section error to the final output is evaluated by sensitivity analysis. This methodology and results can provide the productive information to PIE experimentalists to discuss the discrepancy between calculations and experiments in the AFCI program. Also, the uncertainty information can be utilized for the safety evaluation and validation of fuel burnup codes and nuclear data. 


\section{CONTENTS}

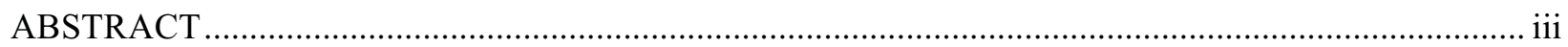

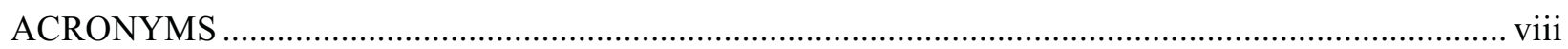

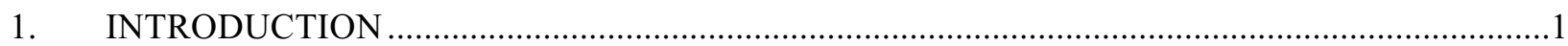

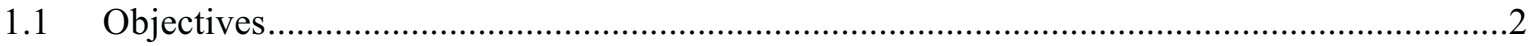

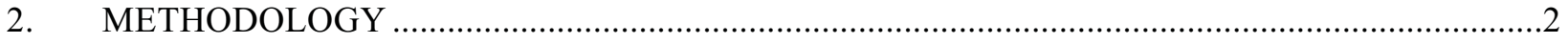

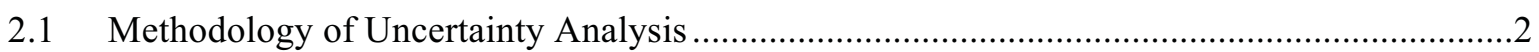

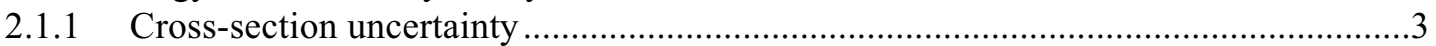

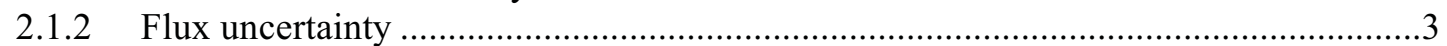

2.1.3 Global Uncertainty Analysis ..........................................................................

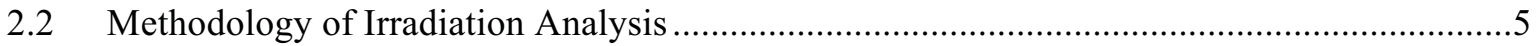

2.2.1 Neutron Flux Normalization Factor ..................................................................

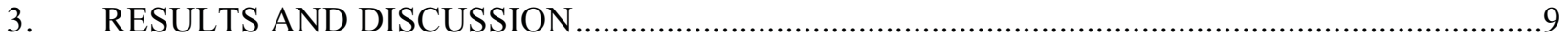

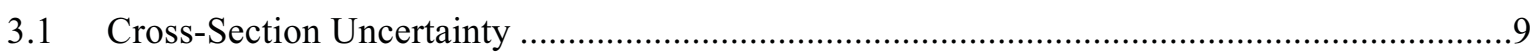

3.2 Depletion and Buildup Calculation Results and Global Uncertainty..................................21

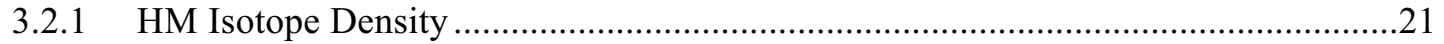

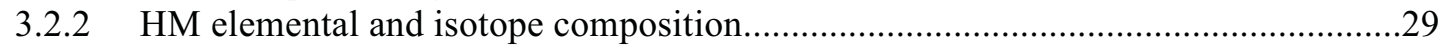

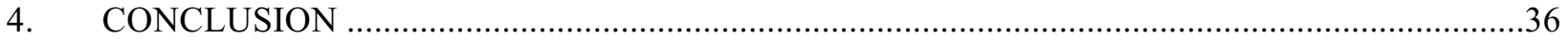

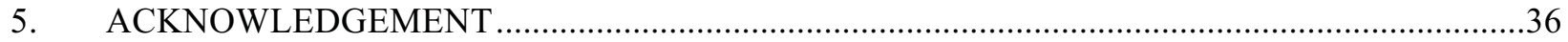

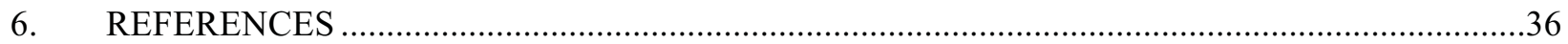

Appendix A—Results of Isolated EFT Model with Boundary Neutron Source $(\Delta \phi=5-10 \%) \ldots \ldots \ldots \ldots \ldots . . .39$

Appendix B —Cross Section and Fission Yield Error of RE Nuclide …..............................................47

\section{FIGURES}

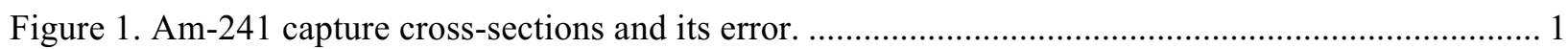

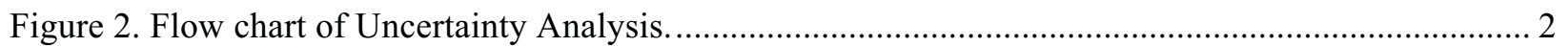

Figure 3. Nuclear Transmutation Chain considered in AFC-2A, 2B fuel analysis. .................................. 5

Figure 4. Horizontal cross-section of EFT with AFC-2A, 2B.............................................................. 6

Figure 5 Vertical cross-section of E2 Capsule with AFC-2A rodlet .......................................................... 7

Figure 6. Neutron Flux in AFC-2A and AFC-2B fuel pin in each rodlet........................................... 8

Figure 7. Neutron Spectrum in AFC-2A. (AFC2A: AFC-2A rodlet 3 fuel pin averaged spectrum

FR: Fast Rector inner core averaged spectrum with $\mathrm{MOX}(\mathrm{U} 0.7, \mathrm{Pu} 0.3)$ with $\mathrm{Na}$ as coolant PWR: PWR pin averaged spectrum with 3.5\% enriched UOX) .................................... 9

Figure 8. U-235 Energy Dependent Cross-Section Error. ${ }^{17}$.................................................................... 10 
Figure 9. U-238 Energy Dependent Reaction Rate in AFC 2A and Cross-Section Error........................ 11

Figure 10. Np-237 Energy Dependent Reaction Rate in AFC 2A and Cross-Section Error..................... 12

Figure 11. Pu-238 Energy Dependent Reaction Rate in AFC 2A and Cross-Section Error...................... 13

Figure 12. Pu-239 Energy Dependent Reaction Rate in AFC 2A and Cross-Section Error..................... 14

Figure 13. Pu-240 Energy Dependent Reaction Rate in AFC 2A and Cross-Section Error..................... 15

Figure 14. Pu-241 Energy Dependent Reaction Rate in AFC 2A and Cross-Section Error..................... 16

Figure 15. Pu-242 Energy Dependent Reaction Rate in AFC 2A and Cross-Section Error..................... 17

Figure 16. Am-241 Energy Dependent Reaction Rate in AFC 2A and Cross-Section Error................... 18

Figure 17. Am-243 Energy Dependent Reaction Rate in AFC 2A and Cross-Section Error.................... 19

Figure 18. Cm-244 Energy Dependent Reactions Rate in AFC 2A and Cross-Section Error................... 20

Figure 19. Sensitivity of cross section error to $\Delta \mathrm{U} 236$, Density Uncertainty .......................................... 23

Figure 20. Sensitivity of cross section error to $\Delta \mathrm{Np} 237$, Density Uncertainty....................................... 23

Figure 21. Sensitivity of cross section error to $\Delta \mathrm{Pu} 238$, Density Uncertainty. ....................................... 24

Figure 22. Sensitivity of cross section error to $\Delta \mathrm{Pu} 242$, Density Uncertainty. ....................................... 24

Figure 23. Sensitivity of cross section error to DAm241, Density Uncertainty. ...................................... 25

Figure 24. Sensitivity of cross section error to DAm243, Density Uncertainty. ...................................... 25

Figure 25. Sensitivity of cross sections error to $\Delta \mathrm{Cm} 242$, Density Uncertainty. ..................................... 26

Figure 26. Sensitivity of cross sections error to $\Delta \mathrm{Cm} 244$, Density Uncertainty. ..................................... 26

Figure 27. Time dependent HM depletion and buildup (AFC-2A rodlet 3) .......................................... 28

Figure 28. Sensitivity of cross section error to Element Composition Uncertainty (AFC 2A

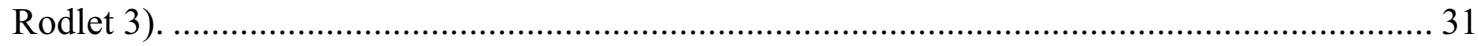

Figure 29. Sensitivity of Cross-section Error to Burnup Uncertainty ...................................................... 35

FigureB-1. Capture cross section error of Nd isotope (Data is derived from Low-Fidelity

Covariance $^{5}$ ).

FigureB-2. Capture cross section error of Nd isotope (Data is derived from Low-Fidelity

Covariance $^{5}$ ).

FigureB-3. Capture cross section error of Ce isotope (Data is derived from Low-Fidelity

Covariance5).

FigureB-4. Capture cross section error of La isotope (Data is derived from Low-Fidelity

Covariance5)

FigureB-5. Comparison of Cumulative Fission Yield between Thermal and Fast Injected Neutron

Database (U-235 and Pu-239, ENDF/VII1). 52

FigureB-6. Cumulative Fission Yield of U-235 and Pu-239 (ENDF/VII1). 


\section{TABLES}

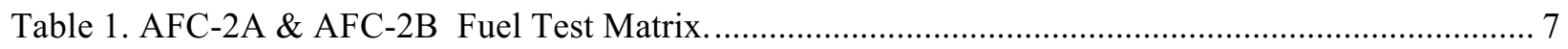

Table 2. Power history in Cycle 140A to 142B for MCWO calculation................................................... 7

Table 3. Effective Cross-Section Relative Uncertainty (1- $\sigma)$ in AFC-2A position................................. 21

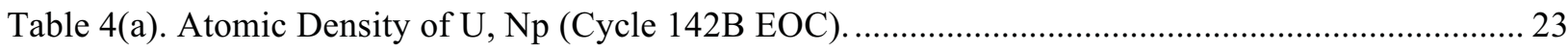

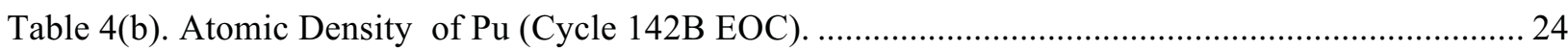

Table 4(c). Atomic Density of Am (Cycle 142B EOC) ......................................................................... 25

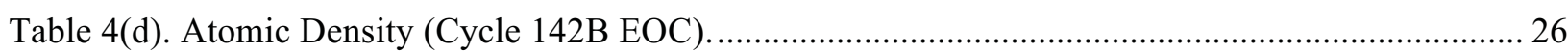

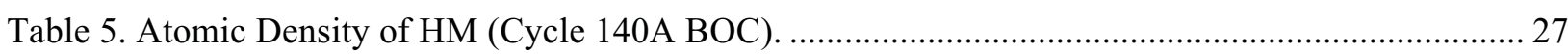

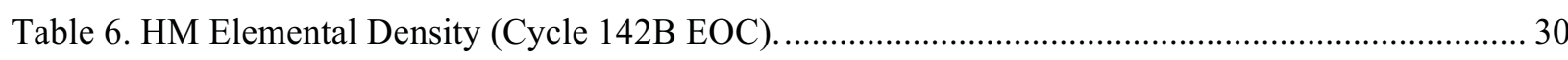

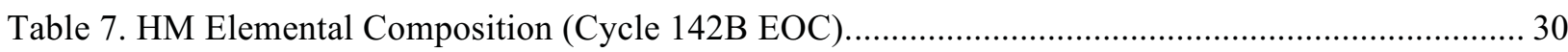

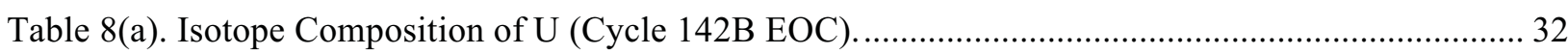

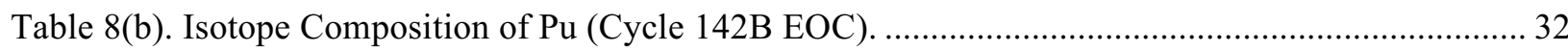

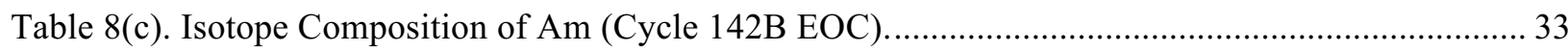

Table 8(d). Isotope Composition of Cm (Cycle 142B EOC) ................................................................... 33

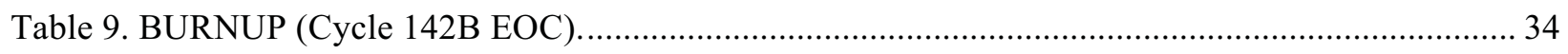

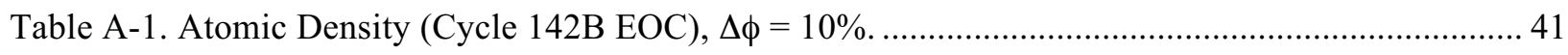

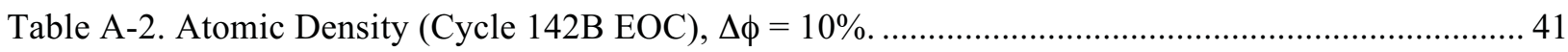

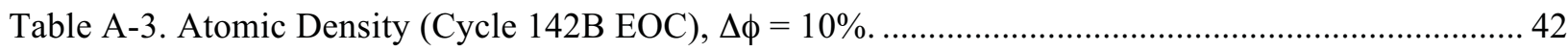

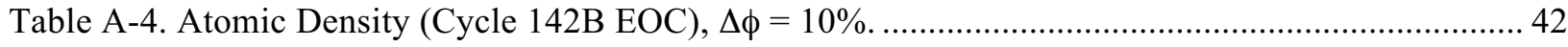

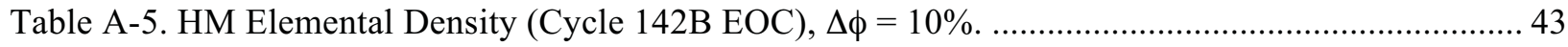

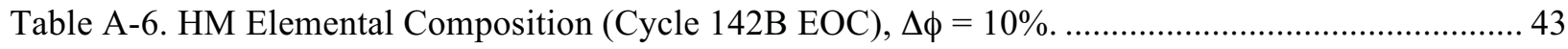

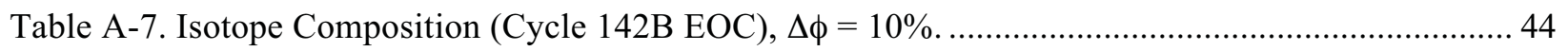

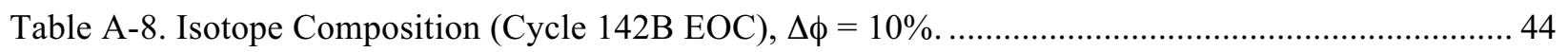

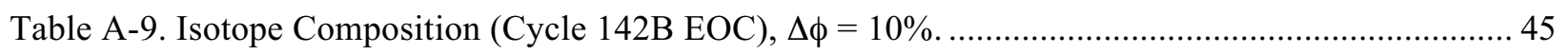

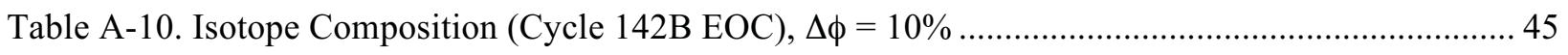

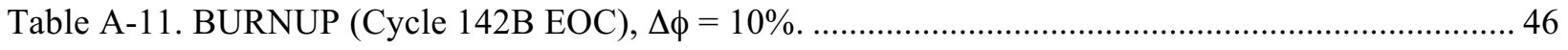




\section{ACRONYMS}

AFC Advanced Fuel Cycle

ATR Advanced Test Reactor

MAs Minor-Actinides

EFPD effective full-power days

EFT East Flux Trap

INL Idaho National Laboratory

JAEA Japan Atomic Energy Agency

PWR Pressurized water reactor

PIE Post Irradiation Examination

HM Heavy Metal

MC Monte-Carlo

MCNP Monte Carlo N-Particle (neutron and photon transport code)

MCWO Monte Carlo with ORIGEN2 (neutron burnup analysis code) 



\section{ATR 2A/2B AS-RUN NEUTRONICS UNCERTAINTY ANALYSIS}

\section{INTRODUCTION}

Neutronics calculations contain several uncertainties such as nuclear data, MC statistics deviation of neutron flux tally, initial mass load, power history, etc. MAs have shortage of measured nuclear data (cross-section, thermal property, etc.) and have a large uncertainty band on nuclear data as shown in Figure 1 . We need to consider the uncertainty caused by nuclear data especially to treat MA including fuel in future reactor designs, safety analysis, irradiation test of fuel concepts for these future reactors, and so on.

Recently covariance matrixes of nuclear data have been included in ENDF/B1, JENDL2, JEF3, TENDL4 and Low-fidelity Covariance5. These contain covariance data of the cross sections for several important nuclides for elastic scattering, inelastic scattering, $(\mathrm{n}, 2 \mathrm{n})$ reactions, radioactive capture and fission (cross section and nubar), in which various approximations were utilized depending on the mass of the target, the neutron energy range, and the neutron reaction.

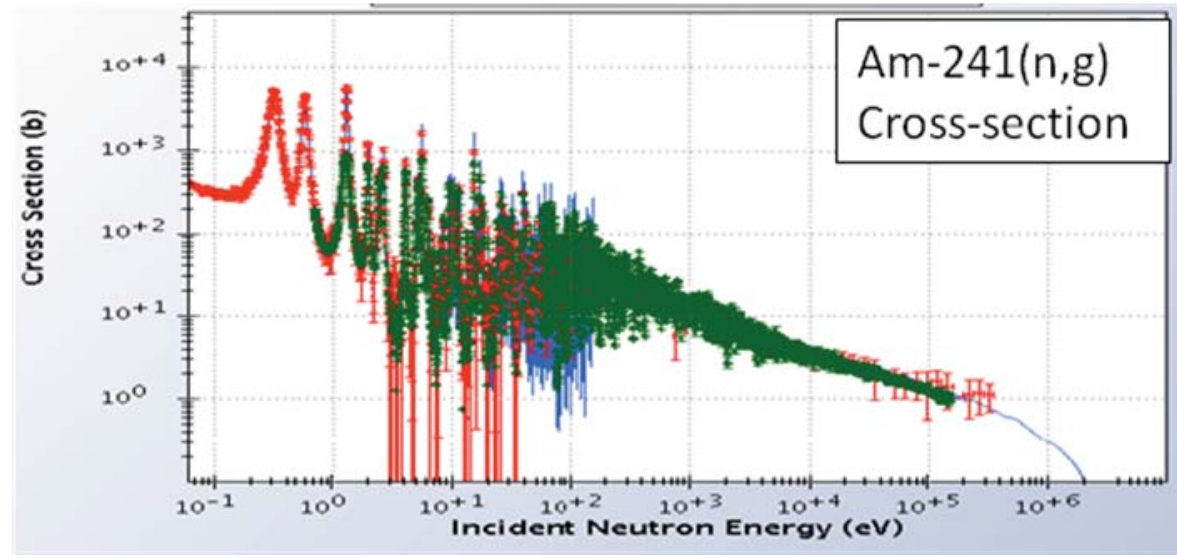

(a) Am-241 capture cross-section with measurement data (ENDF/VII)

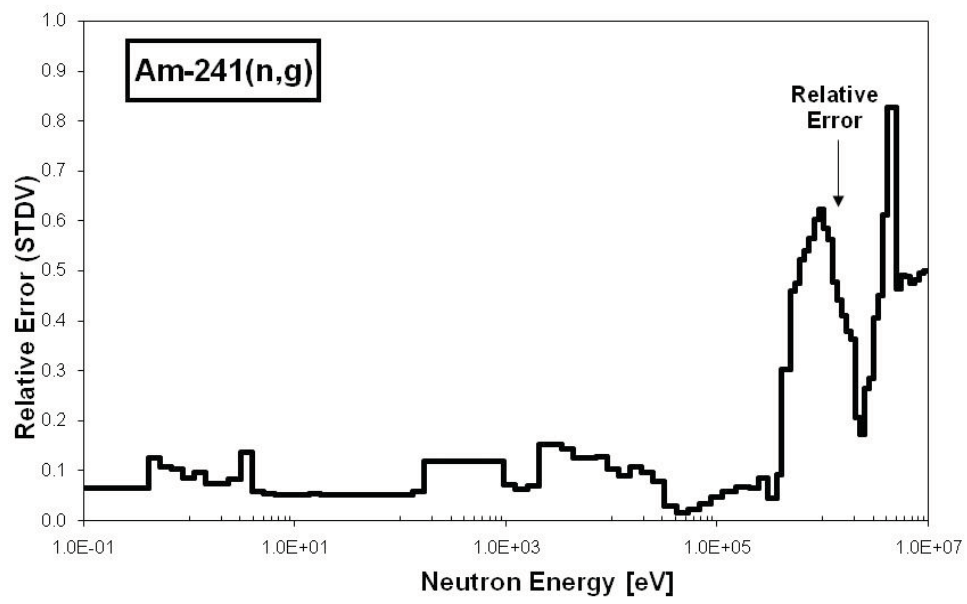

(b) Am-241 capture cross-section error

Figure 1. Am-241 capture cross-sections and its error. 


\subsection{Objectives}

- To establish the method of uncertainty analysis for AFC-2A and $-2 \mathrm{~B}^{6,7,8,9,10}$ irradiation tests

- To evaluate AFC-2A and -2B fuel depletion and buildup with error based on calculation uncertainty

- To provide the uncertainty information related to the neutronics calculation for PIE chemist to assess the difference between the measurement and calculation.

\section{METHODOLOGY}

\subsection{Methodology of Uncertainty Analysis}

The calculation flow chart is shown in Figure 2. In this work, we established the covariance matrix treatment to achieve effective cross-section error in the irradiated AFC-2A and -2B fuel pins, and the global uncertainty treatment in depletion calculation by ORIGEN2. $2^{11}$ and Bateman Equation. We can apply these methodologies to $\mathrm{MCWO}^{12}$ code with interface to the ORIGEN Library. Detailed explanation of each calculation flow chart step is described in next paragraph.

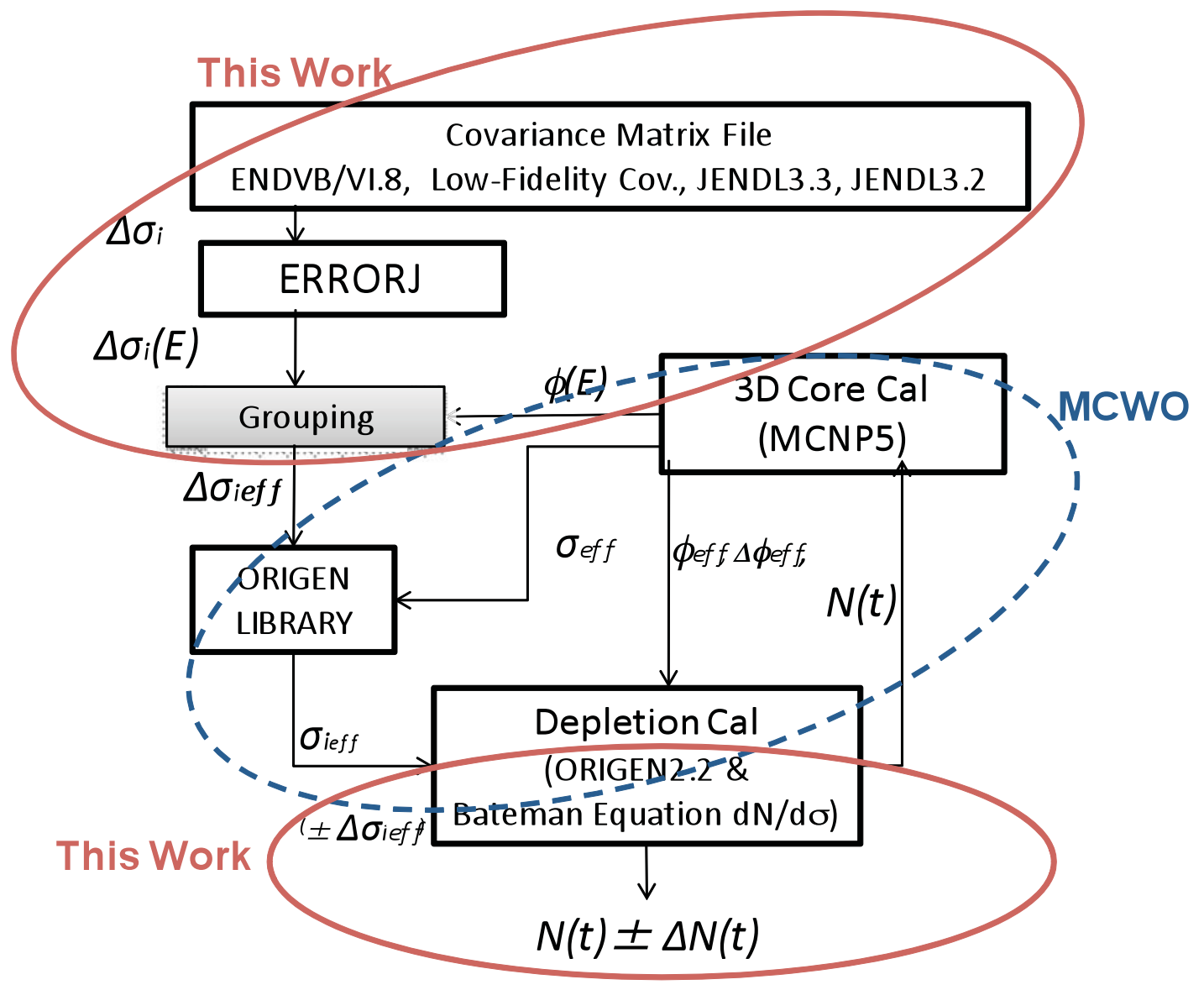

Figure 2. Flow chart of Uncertainty Analysis. 


\subsubsection{Cross-section uncertainty}

When the uncertainties of the nuclear parameters are evaluated in a deterministic manner,

Covariances of the energy-averaged (multi-group) cross sections are necessary. In the present work, ERRORJ2.3 code is utilized for this purpose.

ERRORJ $^{13}$ is a processing code to transform cross section covariance given in the ENDF format into energy-averaged cross section covariance. ERRORJ can process the covariance data of cross sections including resonance parameters, and angular and energy distributions of secondary neutrons. The original ERRORJ code was developed by K. Kosako in $1999^{14}$ based on the ERRORR module of the NJOY code,${ }^{15}$ to generate the covariance of multi-group cross sections from the covariance data given in the nuclear data files. ERRORJ can process the covariance of the cross sections including Breit-Wigner and Reich-Moore resonance parameters, angular and energy distributions of secondary neutrons and the number of neutrons generated by the fission reaction. ERRORJ can also process the covariance of the resonance parameters contained in the 'compact format' recently generated. ERRORJ had been the first code to process such covariance data in the world. Recently, the PUFF-IV ${ }^{16}$ code has been developed at the Oak Ridge National Laboratory. The PUFF-IV code can process the covariance of all the resonance parameters, and it can read the covariance in the compact format. However, the PUFF-IV code cannot treat the covariance of angular and energy distributions of secondary neutrons.

By utilizing ERRORJ, the covariance matrix of each nuclide is converted to the user suggested energy group constants, and by weighting the AFC-2A and $-2 \mathrm{~B}$ neutron spectrum, the effective cross-section error in AFC irradiation fuel pins are created. In the present work, covariance matrixes are extracted from ENDF/VI.8, ENDF/VII, JENDL3.3 and Low-fidelity Covariance for Actinides (U-235, U-238, Pu-238, $\mathrm{Pu}-239, \mathrm{Pu}-240, \mathrm{Pu}-241, \mathrm{Pu}-242, \mathrm{~Np}-237, \mathrm{Am}-241, \mathrm{Am}-243$, and $\mathrm{Cm}-244)$.

Cross-section uncertainty of each nuclide reaction type is calculated by ERRORJ code with 216 energy groups with constant lethargy width. The effective one-group error of cross-section is calculated by

$$
\overline{\Delta \sigma_{e f f}}=\int \frac{\Delta \sigma(E) \phi(E)}{\phi(E)} d E
$$

where $\phi(E)$ represents the energy dependent neutron flux in AFC-2A and -2B fuel pin position and $\Delta \sigma(E)$ is the energy dependent cross-section error provided by the output of ERRORJ.

\subsubsection{Flux uncertainty}

Since there is no measurement data of neutron flux absolute value in the current AFC-2A and -2B irradiation tests, $\mathrm{MC}$ statistical deviation is taken as the flux uncertainty in the full core calculation result provided by Dr. Gray Chang. The MC statistical deviation in the present work was very low (less than $0.5 \%$ ) thus, much lower than cross-section uncertainty. For simplicity, the flux uncertainty is omitted in this work. The calculation with a boundary neutron source for AFC-2A and $-2 \mathrm{~B}$ irradiation position has also been performed and the flux uncertainty was input as $10 \%$ since difference between full core models in flux was 5 to $10 \%$ in AFC-2A and -2B irradiation position. The detailed table sets of these results are included in the Appendix A.

Other uncertainties such as initial load, and power history are not considered in the present report. As built fuel information and as-run power history, provided by MCNP full core analyses, are utilized as fixed values. The unique phenomena of the metal fuel, swelling and elements migration during the irradiation, are also not considered in this work. The depletion and buildup results for each fuel pin are the average value for each pin. 
Rare Earth (RE) depletion and buildup have cross section and fission yield errors. Though as-run depletion and buildup calculation of RE is not performed in this work, the important uncertainty data sets relating to cross section and fission yields are included in the Appendix B.

\subsubsection{Global Uncertainty Analysis}

In nuclear transmutation calculations, we need each nuclide uncertainty to be transported to the final nuclide uncertainty. To evaluate the global uncertainty in transmutation, depletion calculations can be performed using either a numerical approach, such as ORIGEN2.2, or by solving Bateman Equation with perturbation of each parameter.

The mono HM nuclide transmutation from nuclide 1 to nuclide $\mathrm{m}$, the transmutation differential equation, is described with Bateman equation as follows:

$$
\begin{aligned}
& \frac{d N_{1}(t)}{d t}=-\lambda_{1} N_{1}(t) \\
& \frac{d N_{2}(t)}{d t}=\lambda_{i} \cdot N_{1}(t)-\lambda_{2} N_{2}(t) \\
& \frac{d N_{3}(t)}{d t}=\lambda_{i i} \cdot N_{2}(t)-\lambda_{3} \cdot N_{3}(t) \\
& \cdots \\
& \frac{d N_{m}(t)}{d t}=\lambda_{m-i} \cdot N_{m-i}(t)-\lambda_{m} \cdot N_{m}(t)
\end{aligned}
$$

where $\lambda$ is a constant and consists of micro cross-section $\sigma$, neutron flux $\phi$, and decay constant.

The integral solution of the differential equation is

$$
N_{m}(t)=N_{1}^{0} \cdot \prod_{a=i}^{m-i} \lambda_{a} \cdot \sum_{c=1}^{m} e^{-\lambda_{c} \cdot t} \prod_{c \neq d} \frac{1}{\left(\lambda_{d}-\lambda_{c}\right)} .
$$

The increment difference of $N_{m}(\mathrm{t})$ is described as following,

$$
d N_{m}=\frac{\partial N_{m}}{\partial \sigma_{c 1}} d \sigma_{c 1}+\frac{\partial N_{m}}{\partial \sigma_{f 1}} d \sigma_{f 1}+\frac{\partial N_{m}}{\partial \sigma_{(n, 2 n) 1}} d \sigma_{(n, 2 n) 1}+\ldots .+\frac{\partial N_{m}}{\partial \sigma_{(n, 2 n) m}} d \sigma_{(n, 2 n) m}+\frac{\partial N_{m}}{\partial \phi} d \phi
$$

We assume that $N_{m}(\mathrm{t})$ is the linear function with each parameter with a width of $\pm \Delta$, the equation can be converted to the following,

$$
\Delta N_{m}=\sqrt{\left(\frac{\partial N_{m}}{\partial \sigma_{c 1}} \Delta \sigma_{c 1}\right)^{2}+\left(\frac{\partial N_{m}}{\partial \sigma_{f 1}} \Delta \sigma_{f 1}\right)^{2}+\left(\frac{\partial N_{m}}{\partial \sigma_{(n, 2 n) 1}} \Delta \sigma_{(n, 2 n) 1}\right)^{2} \ldots+\left(\frac{\partial N_{m}}{\partial \phi} \Delta \phi\right)^{2}} .
$$

With this equation, we can treat the transportation of each nuclide/reaction uncertainty to the final nuclide as global uncertainty. Depletion calculations can be performed using either a numerical approach such as ORIGEN2.2 or by solving Bateman Equation in Eq. 3, and Eq. 5 can be utilized to evaluate the global uncertainty of nuclide $\mathrm{m}$. 
To calculate the global uncertainty of each nuclide, the EFT irradiation position isolated model with boundary neutron source is applied. Though the isolated model has 5 to $10 \%$ discrepancy in the results of HM depletion and buildup from full core model, it provides good enough information for each differential coefficient in Eq.4 and a good uncertainty ratio. Finally the global uncertainties calculated by the isolated model are applied to the result of full core depletion and buildup calculation in ratio.

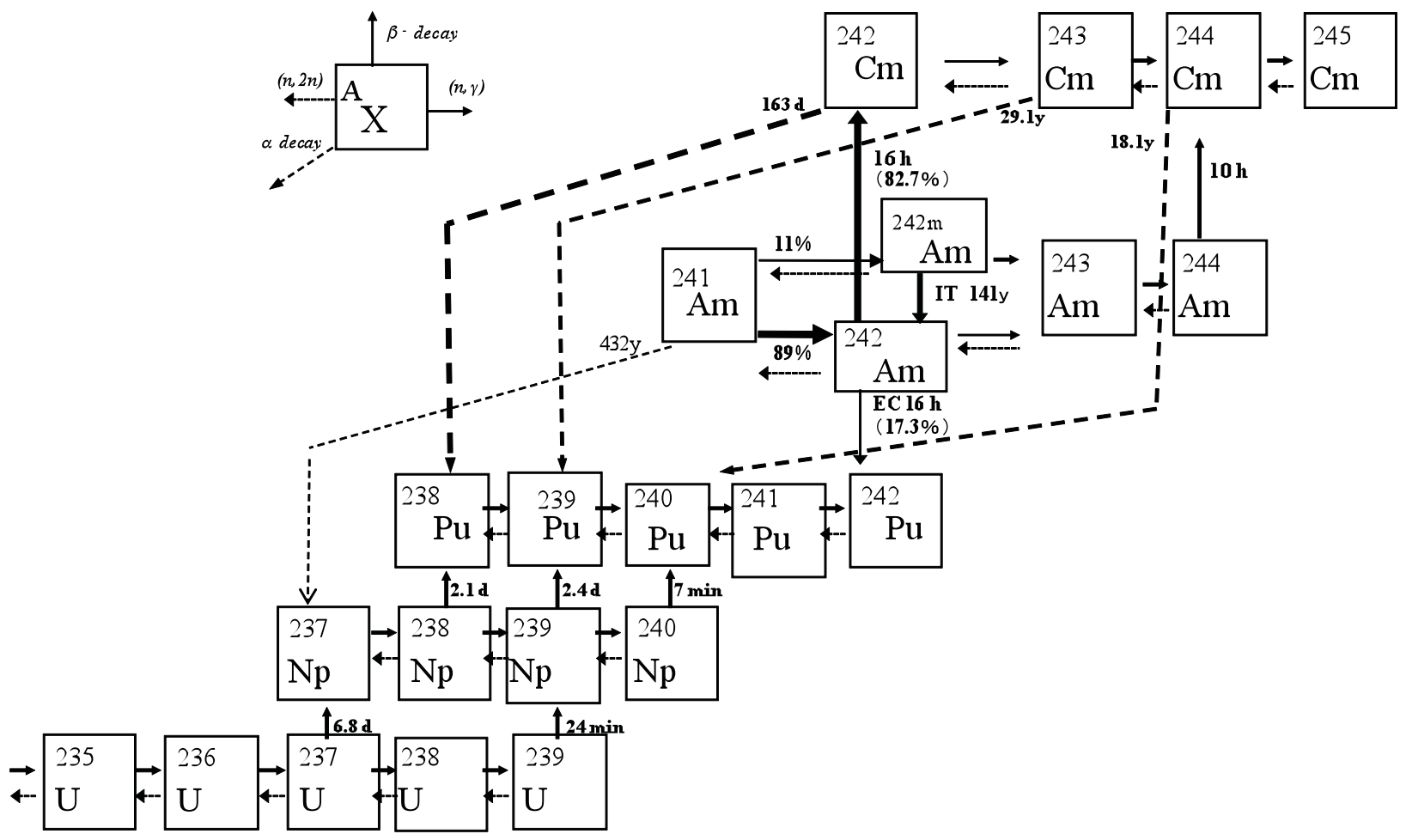

Figure 3. Nuclear Transmutation Chain considered in AFC-2A, 2B fuel analysis.

\subsection{Methodology of Irradiation Analysis}

Irradiation position EFT isolated model with boundary neutron source, SSR option in MCNP5 ver1.3, is applied. The depletion methodology MCWO (using ORIGEN2) was used to evaluate the AFC-2A, $2 \mathrm{~B}$ fuel pin burnup behavior versus EFPD's within the ATR EFT positions.

The standard boundary neutron source was provided from the result of a full core as-run neutronics calculation. The model horizontal and vertical geometrical cross-sections are shown in Figure 4 and Figure 5. The fuel composition and specification are listed in Table 1. To calculate the neutron flux of each calculation cell, the ATR power history (Table 2) and following normalization factors were used to scale the MCNP-calculated flux tallies. 


\subsubsection{Neutron Flux Normalization Factor}

Note, the MCNP f4 tally has units of $1 / \mathrm{cm} 2$ per source neutron.

$$
\begin{aligned}
\text { Flux Normdization Coef f. }\left[n / M W_{\text {core }} / \mathrm{sec}\right] & =\left(\frac{\text { Fis Neutrons }}{F i s}\right)\left(\frac{F i s}{\mathrm{MeV}}\right)\left(\frac{\mathrm{MeV}}{M W_{\text {core }} \cdot \mathrm{sec}}\right) \\
& =\left(\frac{2.43 F \text { is Neutrons }}{F i s}\right)\left(\frac{F i s}{200 \mathrm{MeV}}\right)\left(\frac{6.2415 \times 10^{18} \mathrm{MeV}}{M W_{\text {core }} \cdot \sec }\right) \\
& =7.583 \times 10^{16}\left[\text { Fis Neutrons } / M W_{\text {core }} / \mathrm{sec}\right]
\end{aligned}
$$

By using Eq.6, the neutron fluxes in AFC-2A and -2B position are calculated as Figure 6.

This isolated model is utilized to calculate the global uncertainty of each nuclide. The uncertainty information is applied to the depletion calculation results by full-core ATR model calculation. Though the isolated model has 5 to $10 \%$ discrepancy in the results of HM depletion and buildup from full core model, it still can provide good information in each differential coefficient in Eq.4 and in uncertainty ratio.

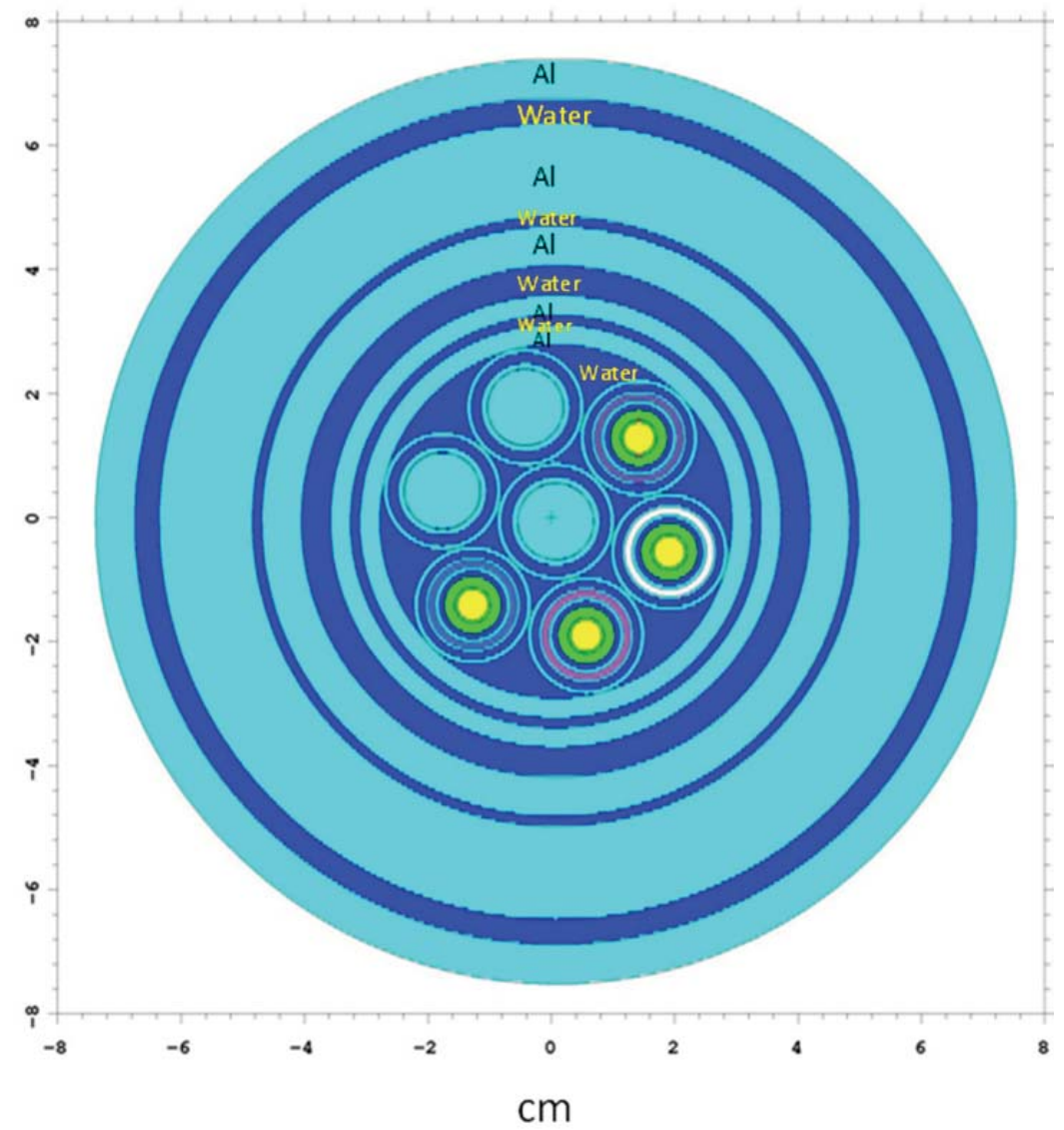

Figure 4. Horizontal cross-section of EFT with AFC-2A, 2B. 


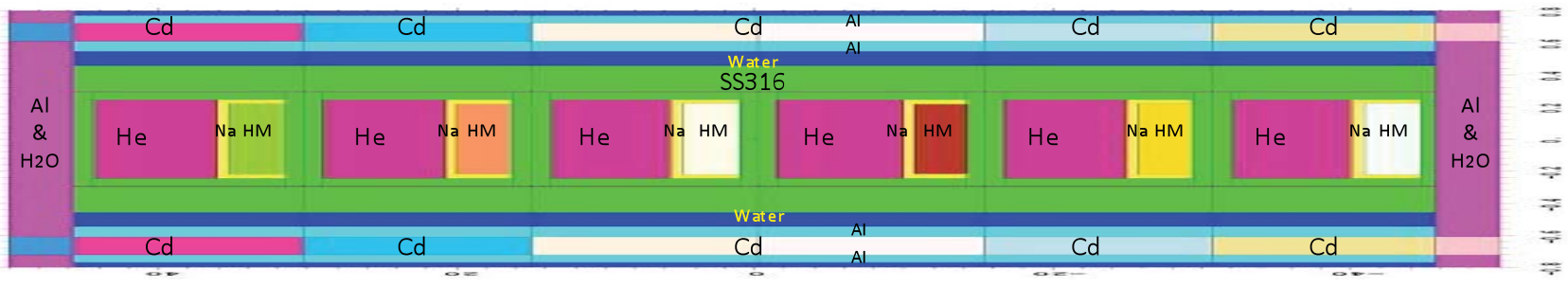

Figure 5 Vertical cross-section of E2 Capsule with AFC-2A rodlet

Table 1. AFC-2A \& AFC-2B Fuel Test Matrix.

\begin{tabular}{|c|c|c|}
\hline Rodlet & Metallic Fuel Alloy $\dagger$ & U-235 En richment \\
\hline $\mathbf{1}$ & U-20Pu-3Am-2Np-15Zr & $93 \%$ \\
\hline $\mathbf{2}$ & U-20Pu-3Am-2Np-1.0RE*-15Zr & $55 \%$ \\
\hline $\mathbf{3}$ & U-20Pu-3Am-2Np-1.5RE*-15Zr & $45 \%$ \\
\hline $\mathbf{4}$ & U-30Pu-5Am-3Np-1.5RE*-20Zr & $55 \%$ \\
\hline $\mathbf{5}$ & U-30Pu-5Am-3Np-1.0RE*-20Zr & $65 \%$ \\
\hline $\mathbf{6}$ & U-30Pu-5Am-3Np-20Zr & $93 \%$ \\
\hline
\end{tabular}

$\dagger$ Alloy composition expressed in weight percent.

$* \mathrm{RE}$ designates rare earth alloy (6\% La, $16 \% \mathrm{Pr}, 25 \% \mathrm{Ce}, 53 \% \mathrm{Nd})$.

Table 2. Power history in Cycle 140A to 142B for MCWO calculation.

\begin{tabular}{|c|c|c|c|}
\hline & $\begin{array}{l}\text { ATR total } \\
\text { Power } \\
\text { [MWth] }\end{array}$ & $\begin{array}{l}\text { Irradiation } \\
\text { Time } \\
\text { [days] }\end{array}$ & $\begin{array}{l}\text { Cooling } \\
\text { Time } \\
\text { [days] }\end{array}$ \\
\hline $140 \mathrm{~A} 1$ & 103.54 & 16 & 0 \\
\hline 140A2 & 103.98 & 18 & 0 \\
\hline $140 \mathrm{~A} 3$ & 104.31 & 12.5 & 0 \\
\hline $140 \mathrm{~A} 4$ & 104.31 & 0 & 15 \\
\hline 140B1 & 102.52 & 12 & 0 \\
\hline 140B2 & 107.32 & 16 & 0 \\
\hline 140B3 & 102.23 & 7.7 & 0 \\
\hline 140B4 & 102.23 & 0 & 10 \\
\hline 141A1 & 105.7 & 18 & 0 \\
\hline $141 \mathrm{~A} 2$ & 105.62 & 10 & 0 \\
\hline $141 \mathrm{~A} 3$ & 105.4 & 4.4 & 0 \\
\hline $141 \mathrm{~A} 4$ & 105.6 & 0 & 55 \\
\hline 142A1 & 112.65 & 16 & 0 \\
\hline 142A2 & 113.88 & 16 & 0 \\
\hline $142 \mathrm{~A} 3$ & 114.79 & 16 & 0 \\
\hline $142 \mathrm{~A} 4$ & 114.07 & 0 & 13 \\
\hline 142B1 & 116.4 & 16 & 0 \\
\hline 142B2 & 116.72 & 13.7 & 0 \\
\hline 142B3 & 119.37 & 22.3 & 0 \\
\hline 142B4 & 116.5 & 0 & 25 \\
\hline
\end{tabular}



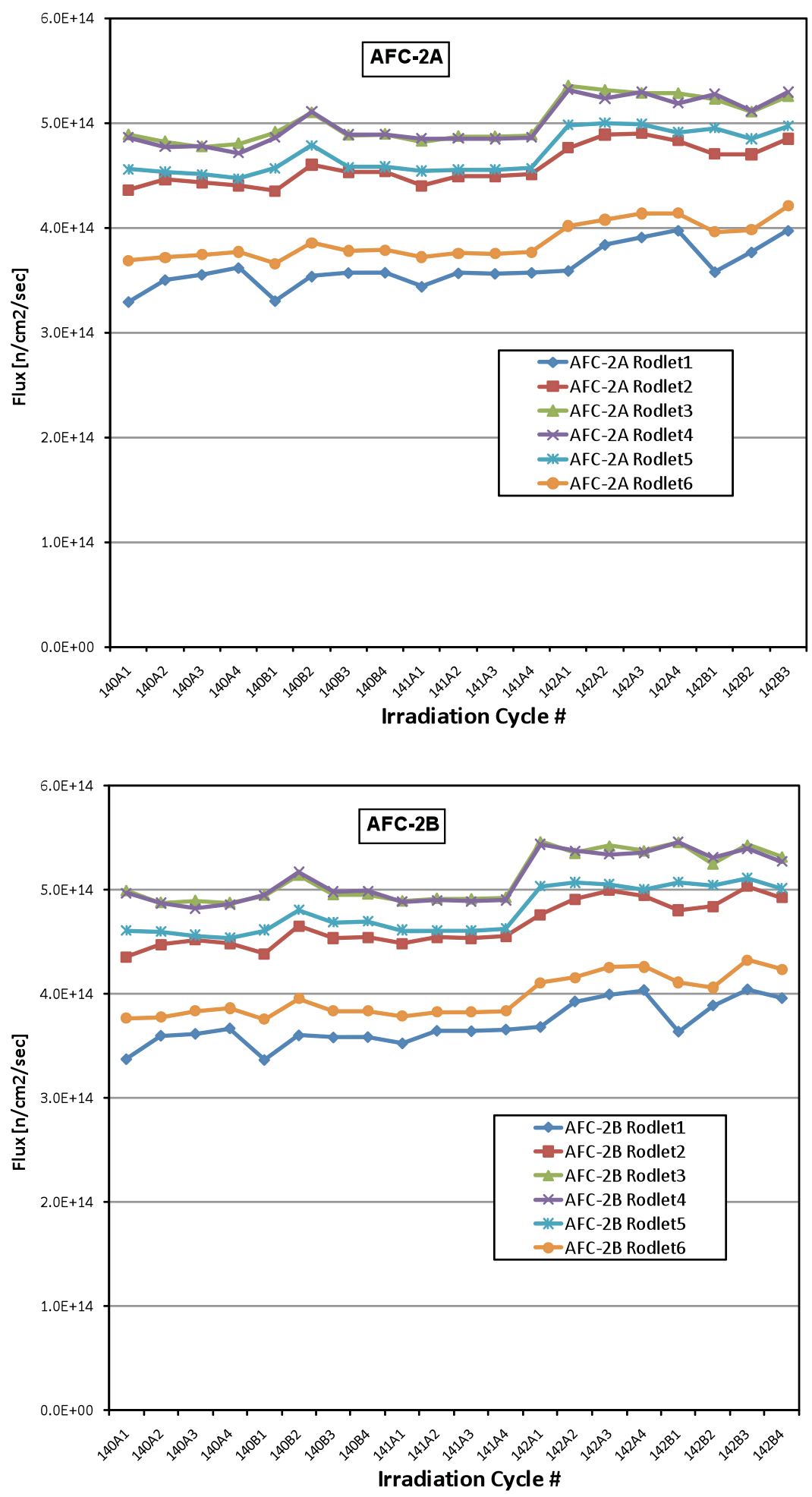

Figure 6. Neutron Flux in AFC-2A and AFC-2B fuel pin in each rodlet. 


\section{RESULTS AND DISCUSSION}

The neutron cross-section error bands evaluated are: U-235, U-238, Np-237, and Pu-238 to Pu-242, Am241, Am-243, and Cm-244. Nuclear reactions whose error data were not available are assumed as following; U-236 cross-section error is assumed to be the same as the U-238 data, Am-242m and Am242 error is assumed the same as Am-241 data, and Cm-242, Cm-243 cross-section error are assumed to be the same as Cm-244 data. Due to the un-reliable (n,2n) cross-section, the (n,2n) cross-section error is assumed as $10 \%$. The neutronics uncertainty analysis results and discussion are presented as follow.

\subsection{Cross-Section Uncertainty}

Cross-section uncertainty of each nuclide reaction type is calculated by ERRORJ code with 216 energy groups with constant lethargy width in relative value $(\%)$. Energy dependent reaction rate in AFC-2A, $2 \mathrm{~B}$ position is also calculated. Since AFC-2A and -2B irradiation positions have a unique neutron spectrum due to $\mathrm{Cd}$ filter, there is no thermal neutron $(<0.5 \mathrm{eV})$ component, $50 \%$ of fast neutron $(>0.1 \mathrm{MeV})$ and $50 \%$ epi-thermal neutron $(0.5 \mathrm{eV}<\mathrm{E}<0.1 \mathrm{MeV})$ as shown in Figure 7. For comparison, neutron spectrum of typical fast reactors and PWR are also drawn in the figure. As shown in the figure, AFC-2A and -2B position neutron spectrum contains more epithermal neutron than typical fast reactor, epi-thermal neutrons are very important for the capture reaction and fissile nuclide fission reactions.

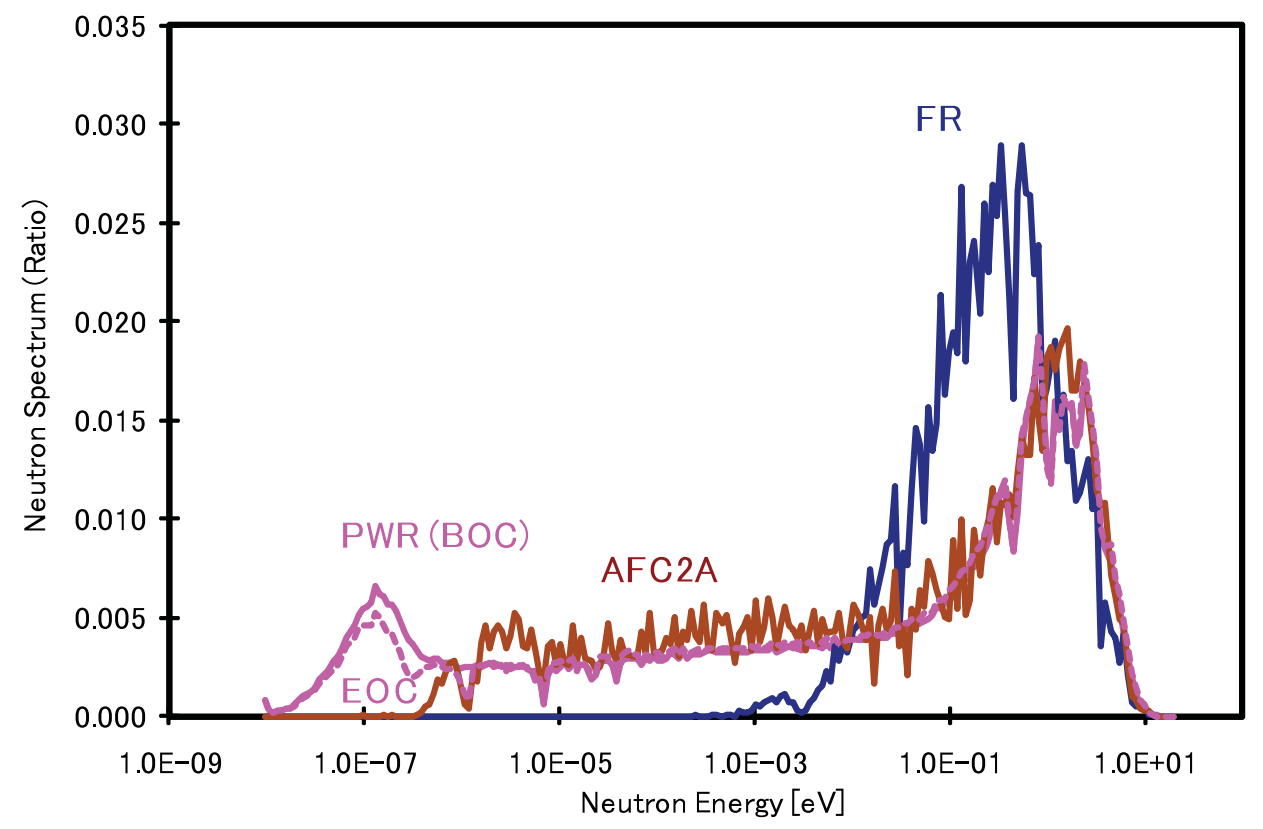

Figure 7. Neutron Spectrum in AFC-2A. (AFC2A: AFC-2A rodlet 3 fuel pin averaged spectrum FR: Fast Rector inner core averaged spectrum with $\mathrm{MOX}(\mathrm{U} 0.7, \mathrm{Pu} 0.3)$ with $\mathrm{Na}$ as coolant PWR: PWR pin averaged spectrum with $3.5 \%$ enriched UOX)

Cross-section uncertainty of each nuclide, reaction type (capture, fission and $\mathrm{n}, 2 \mathrm{n}$ if nuclear data available) is shown in Figure 9 to Figure 18. Energy dependent reaction rate is also shown in the same figures to evaluate the importance of energy to the cross-section error. Because of the similarity of the neutron spectrum in AFC-2A and AFC-2B fuel pins, only AFC-2A position spectrum is used for the analysis as being representative of $\mathrm{AFC}-2 \mathrm{~A}$ and $\mathrm{AFC}-2 \mathrm{~B}$ spectrum. 
Uranium-235 cross-section errors are shown in Figure 9. The covariance data of U-235 is derived from JENDL3.3. Since there is abundant measurement data for U-235, the effective one-group capture crosssection error in $\mathrm{AFC}-2 \mathrm{~A}$ is calculated as $1 \%$. In neutron fission reaction, the effective one-group capture cross-section error in AFC- $2 \mathrm{~A}$ is calculated as $1 \%$. In $\mathrm{n}, 2 \mathrm{n}$ reaction, the effective one-group $(\mathrm{n}, 2 \mathrm{n})$ crosssection error in AFC-2A is calculated as $10 \%$.

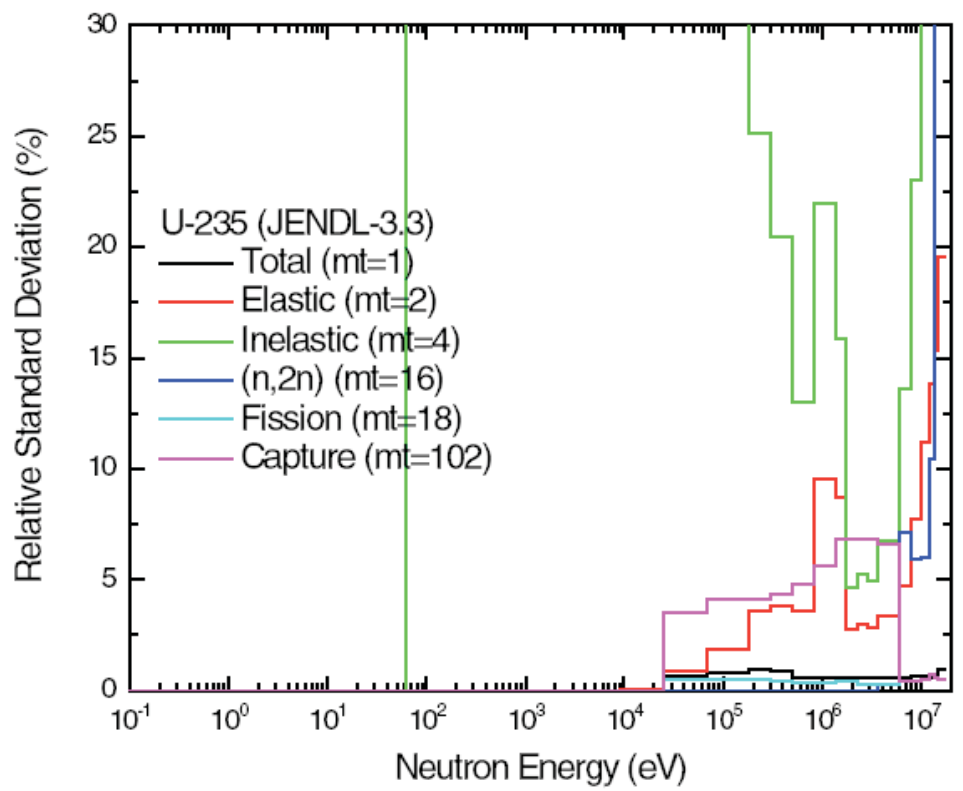

Figure 8. U-235 Energy Dependent Cross-Section Error. ${ }^{17}$

Uranium-238 cross-section error and reaction rate in AFC-2A are shown in Figure 9. Neutron capture cross-section error and reaction rate are shown in Figure 9(a), fission cross-section error and reaction rate are shown in Figure 9(b), n,2n cross-section error and reaction rate are shown in Figure 9(c). The covariance data of U-238 is derived from JENDL3.3. In neutron capture reaction, due to the unique neutron spectrum in AFC 2A, all the neutron capture reactions occurred with epi-thermal neutrons in resonance capture. Since there is abundant measurement data for U-238, capture cross-section error in epi-thermal energy range is as low as about $3 \%$, and the effective one-group capture cross-section error in AFC-2A is calculated as 3\%. In neutron fission reaction, due to the threshold fission cross-section of U238 at about $1 \mathrm{MeV}$, all the neutron fission reactions occurred with fast neutrons, the effective one-group capture cross-section error in AFC-2A is calculated as $1 \%$. In $n, 2 n$ reaction, the effective one-group $(\mathrm{n}, 2 \mathrm{n})$ cross-section error in $\mathrm{AFC}-2 \mathrm{~A}$ is calculated as $3 \%$. 


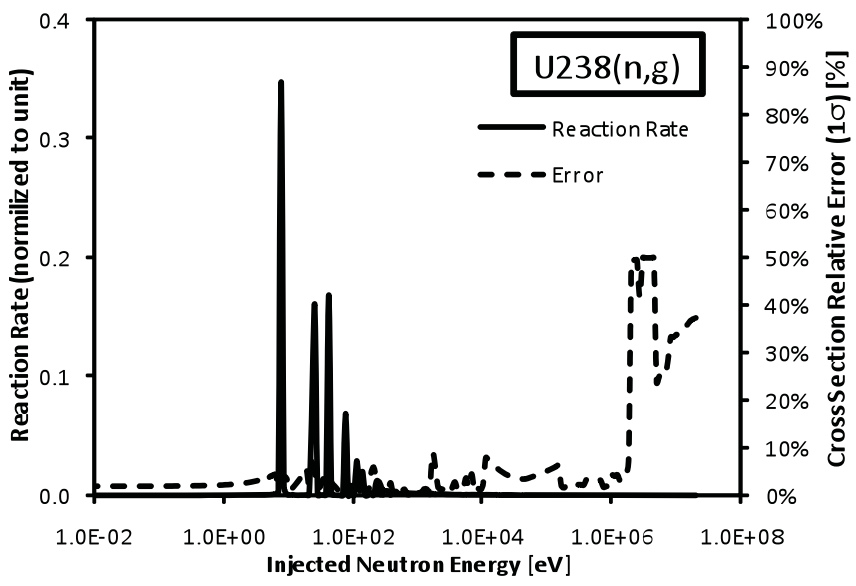

(a) Capture Reaction Rate and Cross Section Error

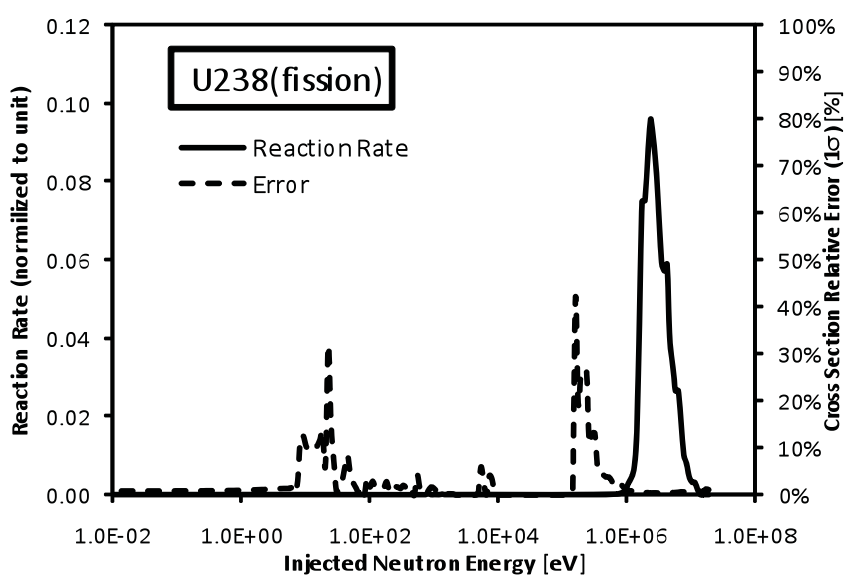

(b) Fission Reaction Rate and Cross Section Error

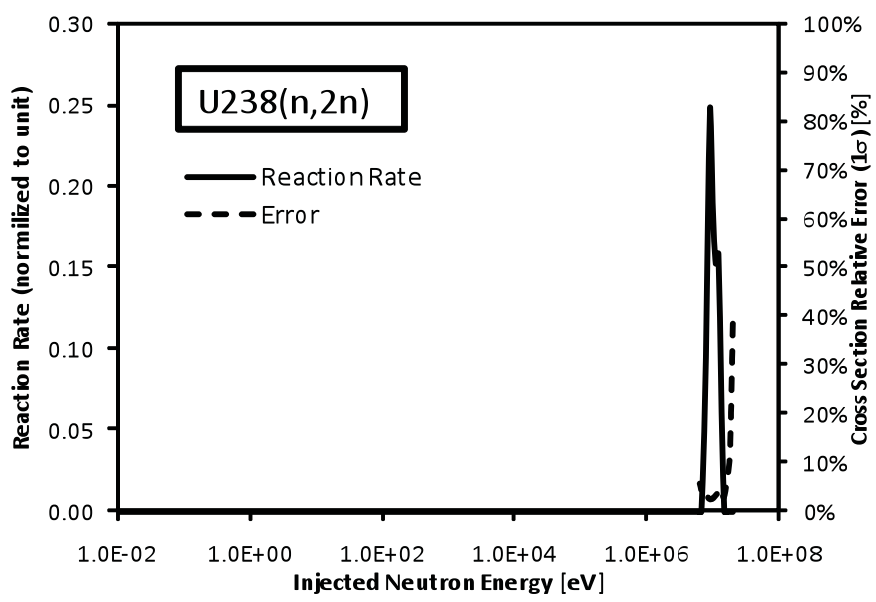

(c) $(n, 2 n)$ Reaction Rate and Cross Section Error

Figure 9. U-238 Energy Dependent Reaction Rate in AFC 2A and Cross-Section Error. 
Neptunium-237 cross-section error and reaction rate in AFC-2A are shown in Figure 10. Neutron capture cross-section error and reaction rate are shown in Figure 10(a), fission cross-section error and reaction rate are shown in Figure 10(b). The covariance data of Np-237 is derived from both JENDL3.3 and Lowfidelity Covariance. The figures and tables in the present report illustrate evaluations with JENDL3.3 as representative. In neutron capture reaction, due to the unique neutron spectrum in AFC $2 \mathrm{~A}$, almost all the neutron capture reactions are resonance capture with epi-thermal neutrons around $0.6 \mathrm{eV}$ and $1.7 \mathrm{eV}$. The effective one-group capture cross-section error in AFC-2A is calculated as $5 \%$. The neutron fission reaction, due to large fast fission cross-section of $\mathrm{Np}-237$ in hundreds $\mathrm{KeV}$ and resonance fission in tens to hundreds $\mathrm{eV}$, occur with mostly fast neutrons and partly epithermal neutrons, the effective one-group fission cross-section error in $\mathrm{AFC}-2 \mathrm{~A}$ is calculated as $3 \%$.

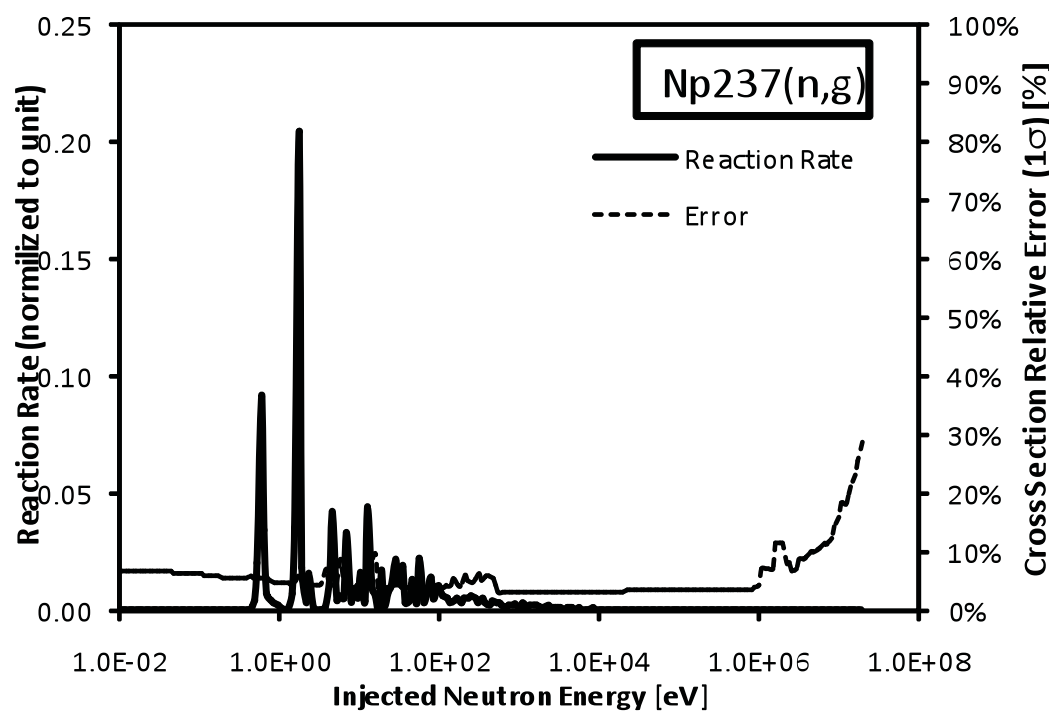

(a) Capture Reaction Rate and Cross Section Error

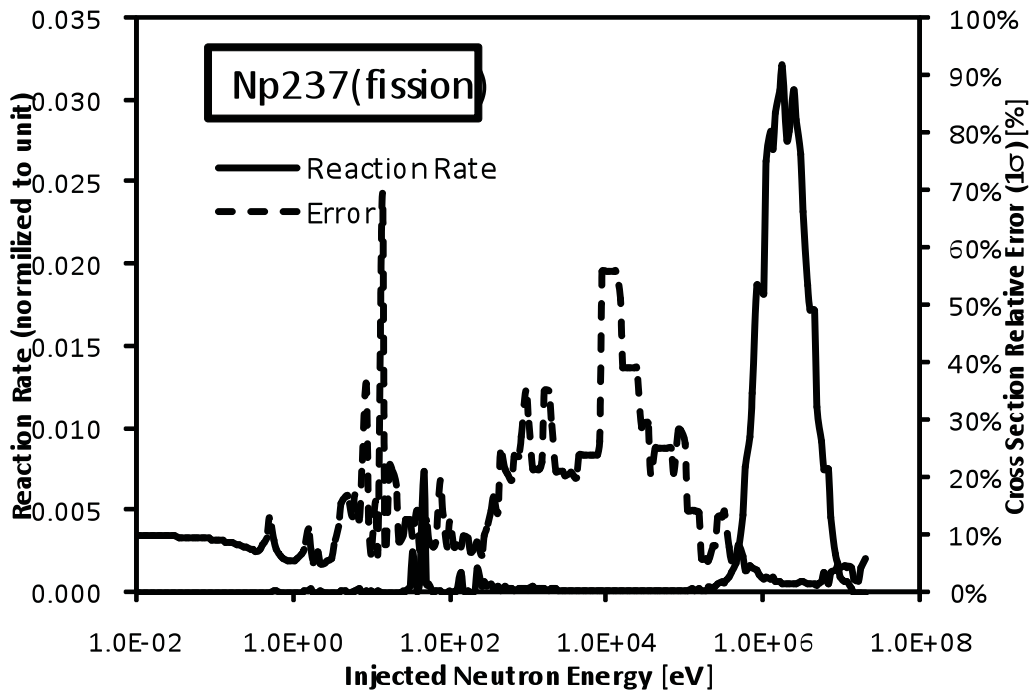

(b) Fission Reaction Rate and Cross Section Error

Figure 10. Np-237 Energy Dependent Reaction Rate in AFC 2A and Cross-Section Error. 
Plutonium-238 cross-section error and reaction rate in AFC-2A are shown in Figure 11. Neutron capture cross-section error and reaction rate are shown in Figure 11(a), fission cross-section error and reaction rate are shown in Figure 11(b). The covariance data of Pu-238 is derived from both JENDL3.3 and Lowfidelity Covariance The figures and tables in the present report illustrate JENDL3.3 data as representative. In neutron capture reaction, due to the unique neutron spectrum in $\mathrm{AFC} 2 \mathrm{~A}$, almost all the neutron capture reactions are occurred with epi-thermal neutron in resonance capture. Due to the large uncertainty in broader energy range, the effective one-group capture cross-section error in AFC-2A is calculated as $27 \%$. In neutron fission reaction, due to fast fission and large resonance fission in one to hundreds $\mathrm{eV}$, neutron fission reactions are occurred broadly with from epithermal to fast neutron, the effective onegroup fission cross-section error in AFC-2A is calculated as $18 \%$.

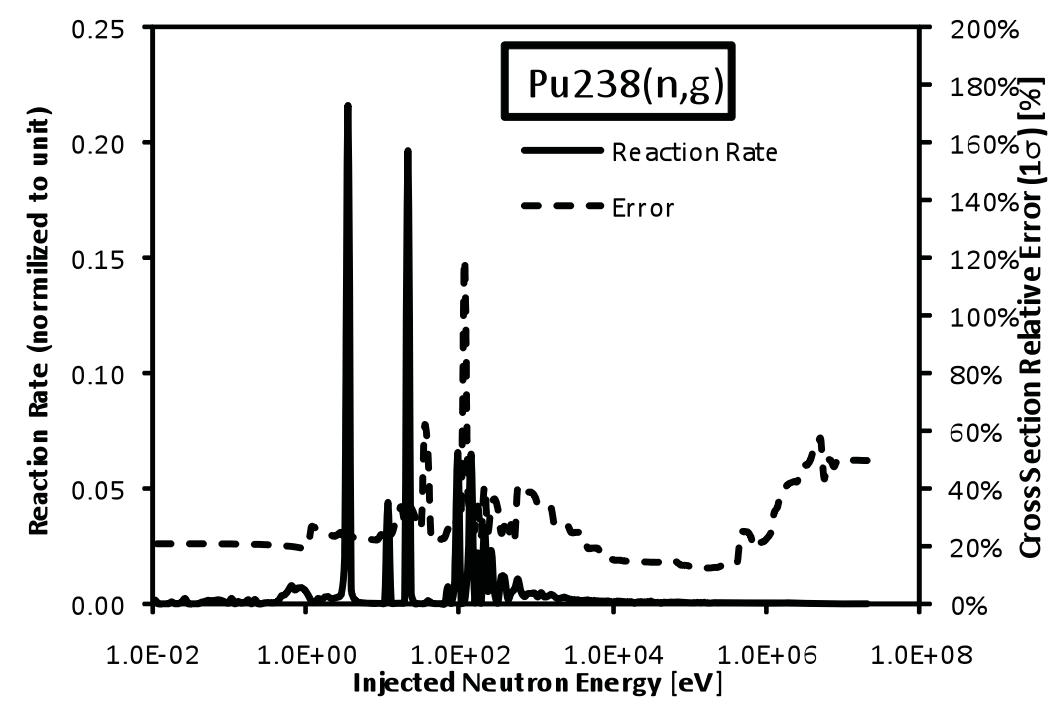

(a) Capture Reaction Rate and Cross Section Error

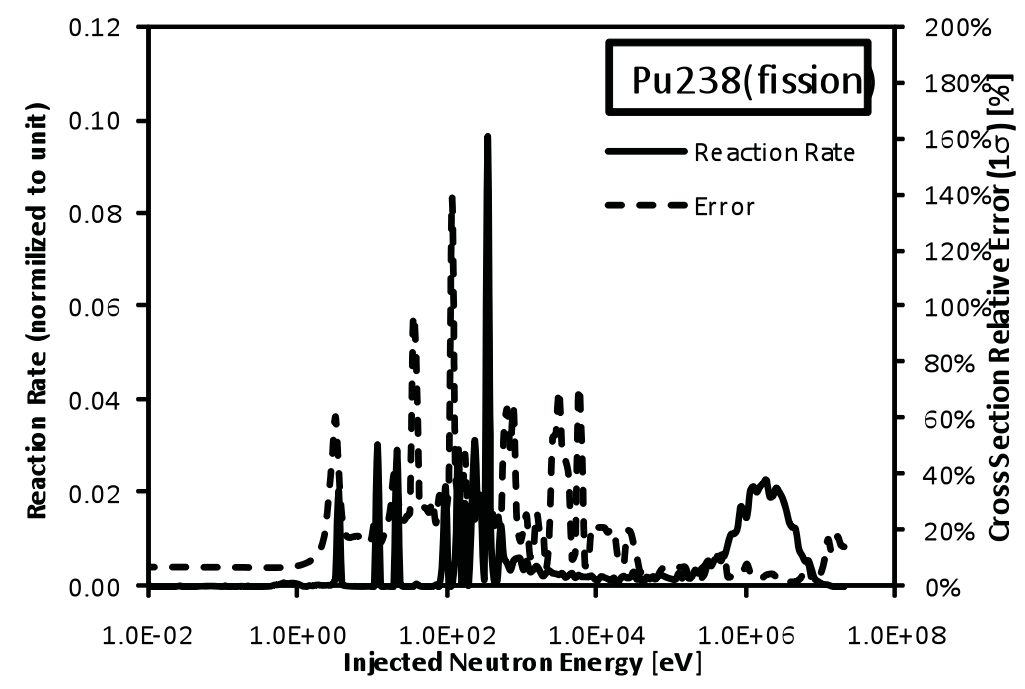

(b) Fission Reaction Rate and Cross Section Error

Figure 11. Pu-238 Energy Dependent Reaction Rate in AFC 2A and Cross-Section Error. 
Plutonium-239 cross-section error and reaction rate in AFC-2A are shown in Figure 12. Neutron capture cross-section error and reaction rate are shown in Figure 12(a), fission cross-section error and reaction rate are shown in Figure 12(b), n,2n cross-section error and reaction rate are shown in Figure 12(c). The covariance data of $\mathrm{Pu}-239$ is derived from JENDL3.3. For neutron capture reaction, due to the unique neutron spectrum in AFC 2A, almost all the neutron capture reactions occurred with epi-thermal neutrons in resonance capture. The effective one-group capture cross-section error in AFC-2A is calculated as 4\%. Neutron fission reactions, due to fast fission and large resonance fission, occur broadly from epithermal to fast neutron energy. The effective one-group fission cross-section error in AFC-2A is calculated as $6 \%$. In $\mathrm{n}, 2 \mathrm{n}$ reaction, due to the large error in nuclear data, the effective one-group $(\mathrm{n}, 2 \mathrm{n})$ cross-section error in AFC-2A is calculated as $205 \%$. However, the one-group $(\mathrm{n}, 2 \mathrm{n})$ cross-section is very small in ATR test position. Its impact on the calculated MA inventories is negligible.

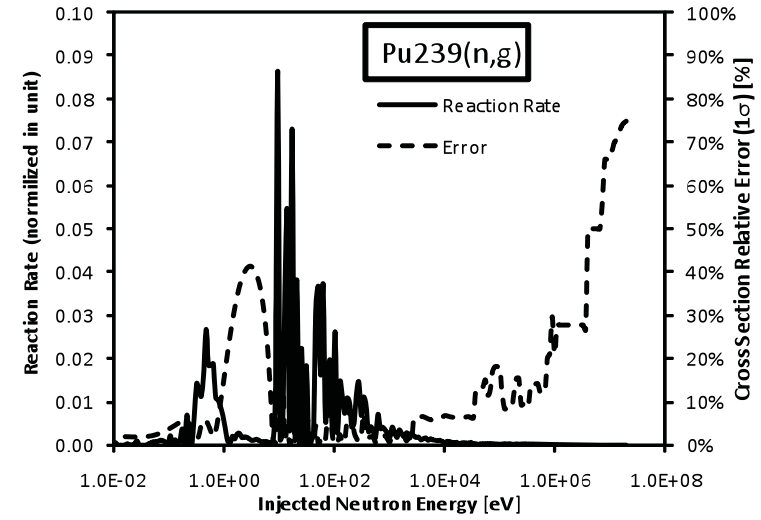

(a) Capture Reaction Rate and Cross Section Error

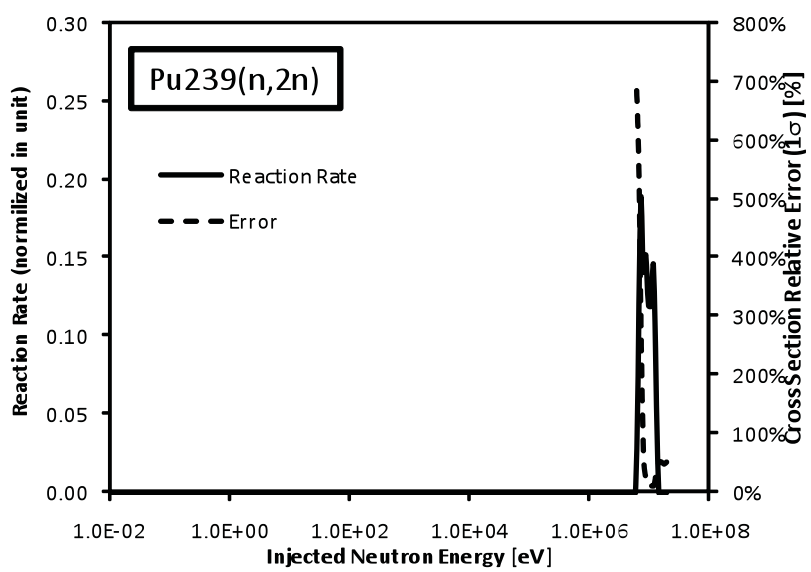

(c) $(n, 2 n)$ Reaction Rate and Cross Section Error

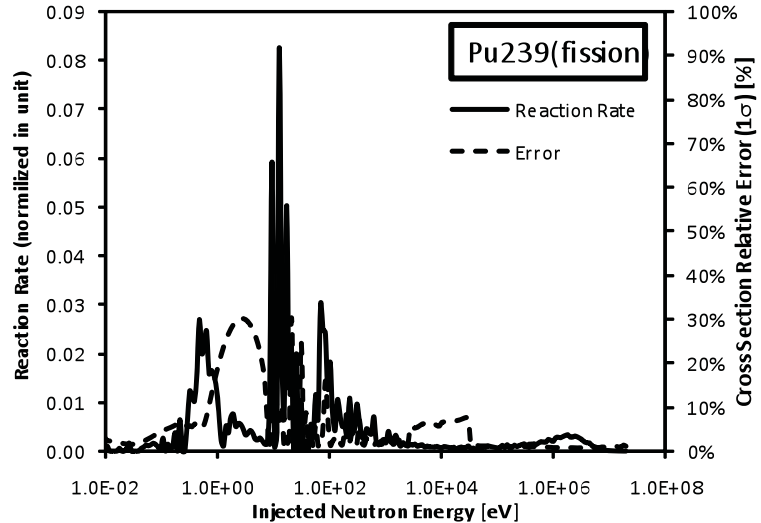

(b) Fission Reaction Rate and Cross Section Error

Figure 12. Pu-239 Energy Dependent Reaction Rate in AFC 2A and Cross-Section Error. 
Plutonium-240 cross-section error and reaction rate in AFC-2A are shown in Figure 13. Neutron capture cross-section error and reaction rate are shown in Figure 13(a), fission cross-section error and reaction rate are shown in Figure 13(b), n,2n cross-section error and reaction rate are shown in Figure 13(c). The covariance data of $\mathrm{Pu}-240$ is derived from both JENDL3.3 and Low-Fidelity Covariance, while figures and tables in the present report show evaluations with JENDL3.3 as representative. In neutron capture reaction, due to the unique neutron spectrum in AFC 2A, almost all the neutron capture reactions occurred with epi-thermal neutrons in about $1 \mathrm{eV}$ resonance capture. The effective one-group capture cross-section error in AFC-2A is calculated as $2 \%$. In the neutron fission reaction, due to large fast fission and partly resonance fission, reactions occurred with mostly fast neutrons and partly epi-thermal neutrons. The effective one-group fission cross-section error in AFC-2A is calculated as $1 \%$. In $\mathrm{n}, 2 \mathrm{n}$ reaction, due to the large error in nuclear data, the effective one-group (n,2n) cross-section error in AFC-2A is calculated as $25 \%$.

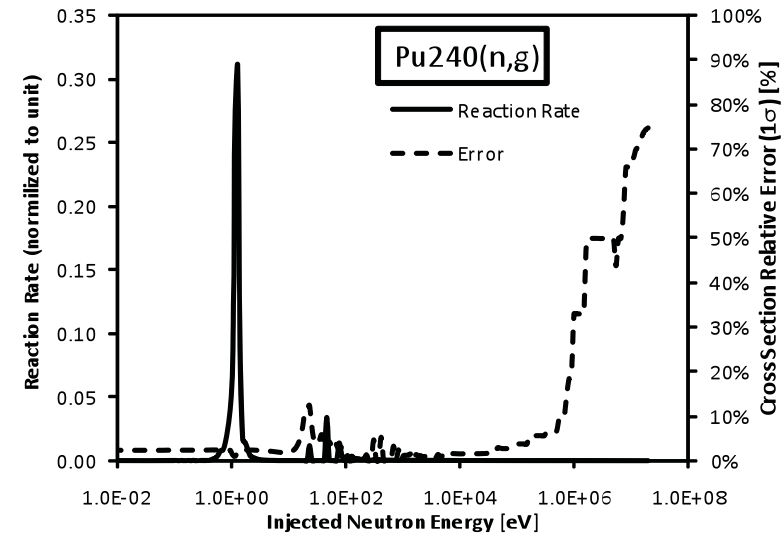

(a) Capture Reaction Rate and Cross Section Error

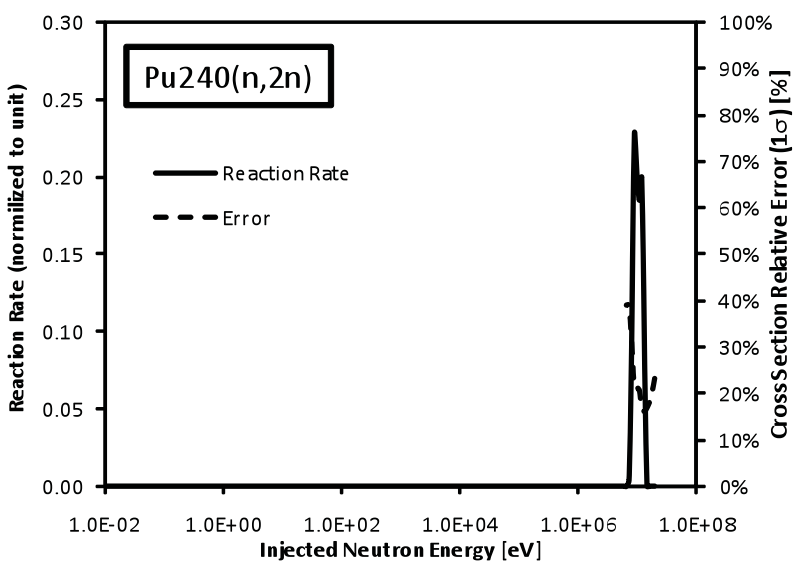

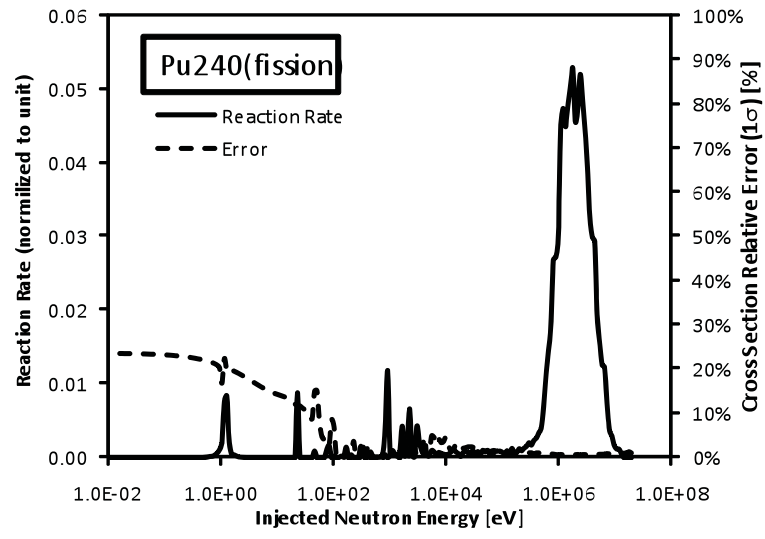

(b) Fission Reaction Rate and Cross Section Error

(c) $(\mathrm{n}, 2 \mathrm{n})$ Reaction Rate and Cross Section Error

Figure 13. Pu-240 Energy Dependent Reaction Rate in AFC 2A and Cross-Section Error. 
Plutonium-241 cross-section error and reaction rate in AFC-2A are shown in Figure 14. Neutron capture cross-section error and reaction rate are shown in Figure 14(a), fission cross-section error and reaction rate are shown in Figure 14(b), n,2n cross-section error and reaction rate are shown in Figure 14(c). The covariance data of Pu-241 is derived from both JENDL3.3 and Low-Fidelity Covariance, and figures and tables in the present report present evaluations with JENDL3.3 as representative. In neutron capture reaction, due to the unique neutron spectrum in AFC $2 \mathrm{~A}$, almost all the neutron capture reactions occurred with epi-thermal neutrons in resonance capture. The effective one-group capture cross-section error in AFC- $2 \mathrm{~A}$ is calculated as $0.5 \%$. Neutron fission reaction, due to fast fission and large resonance fission, occurred broadly with epithermal to fast neutrons. The effective one-group fission cross-section error in AFC-2A is calculated as $0.3 \%$. In $\mathrm{n}, 2 \mathrm{n}$ reaction, due to the large error in nuclear data, the effective onegroup $(\mathrm{n}, 2 \mathrm{n})$ cross-section error in AFC-2A is calculated as $5 \%$.

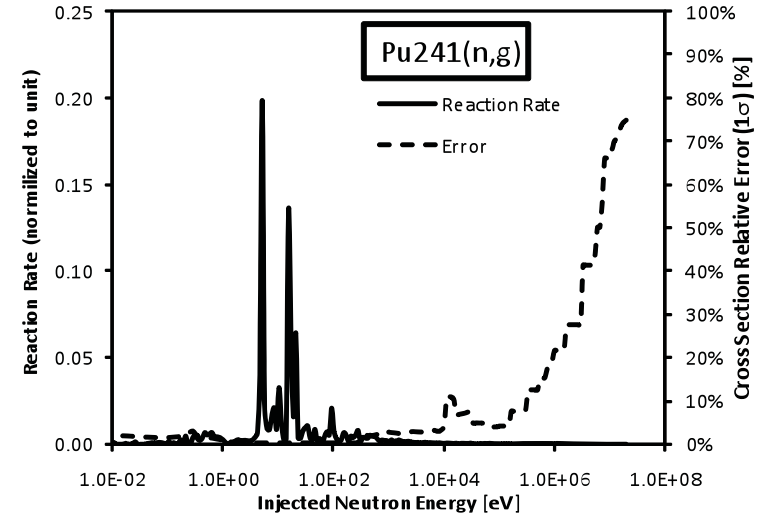

(a) Capture Reaction Rate and Cross Section Error

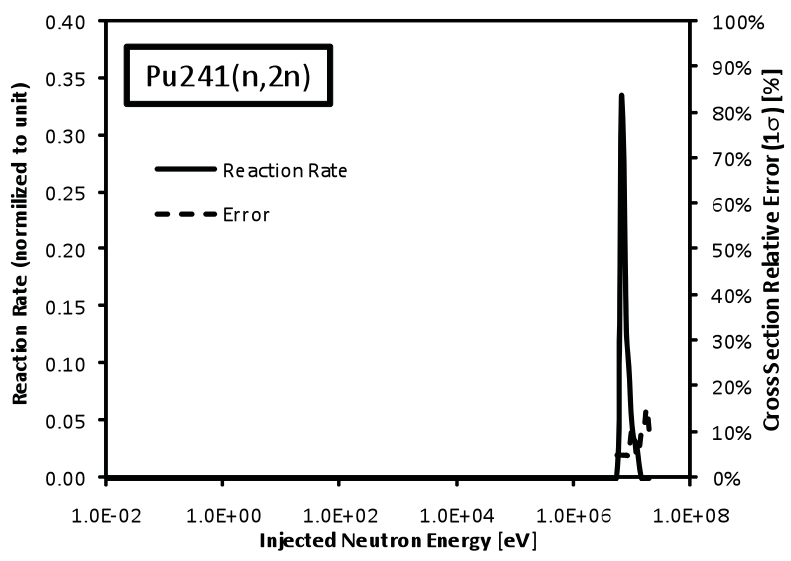

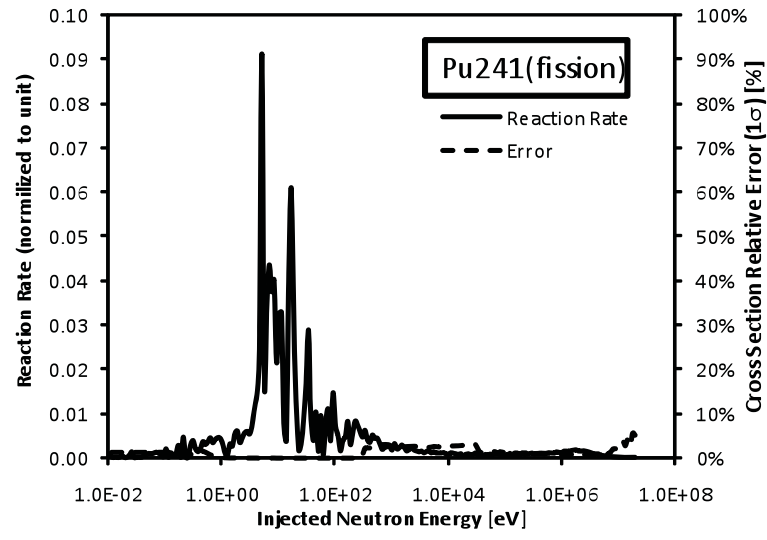

(b) Fission Reaction Rate and Cross Section Error

(c) $(\mathrm{n}, 2 \mathrm{n})$ Reaction Rate and Cross Section Error

Figure 14. Pu-241 Energy Dependent Reaction Rate in AFC 2A and Cross-Section Error. 
Plutonium-242 cross-section error and reaction rate in AFC-2A are shown in Figure 15. Neutron capture cross-section error and reaction rate are shown in Figure 15(a), fission cross-section error and reaction rate are shown in Figure 15(b). The covariance data of Pu-240 is derived from both JENDL3.3 and LowFidelity Covariance, and figures and tables in the present report show evaluations with JENDL3.3 as representative. In neutron capture reaction, due to the unique neutron spectrum in AFC $2 \mathrm{~A}$, almost all the neutron capture reactions occurred with epi-thermal neutron, $3 \mathrm{eV}$, resonance capture. The effective onegroup capture cross-section error in AFC-2A is calculated as 4\%. Neutron fission reaction, due to large fast fission and partial resonance fission, occurred with mostly fast neutron and partially epi-thermal neutrons, the effective one-group fission cross-section error in AFC-2A is calculated as $4 \%$.

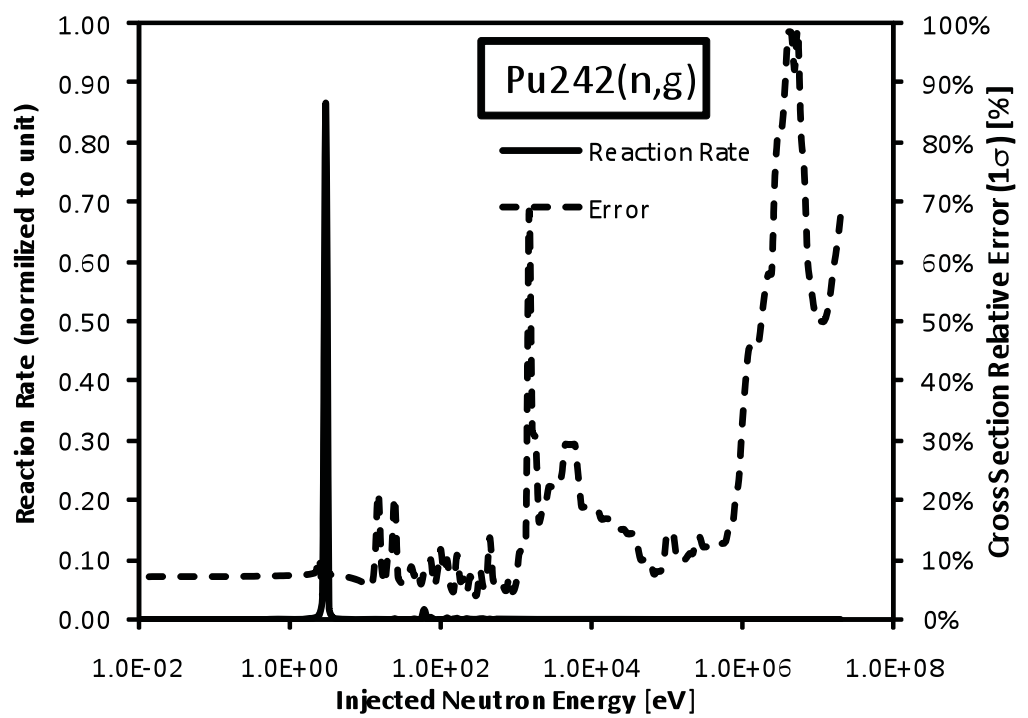

(a) Capture Reaction Rate and Cross Section Error

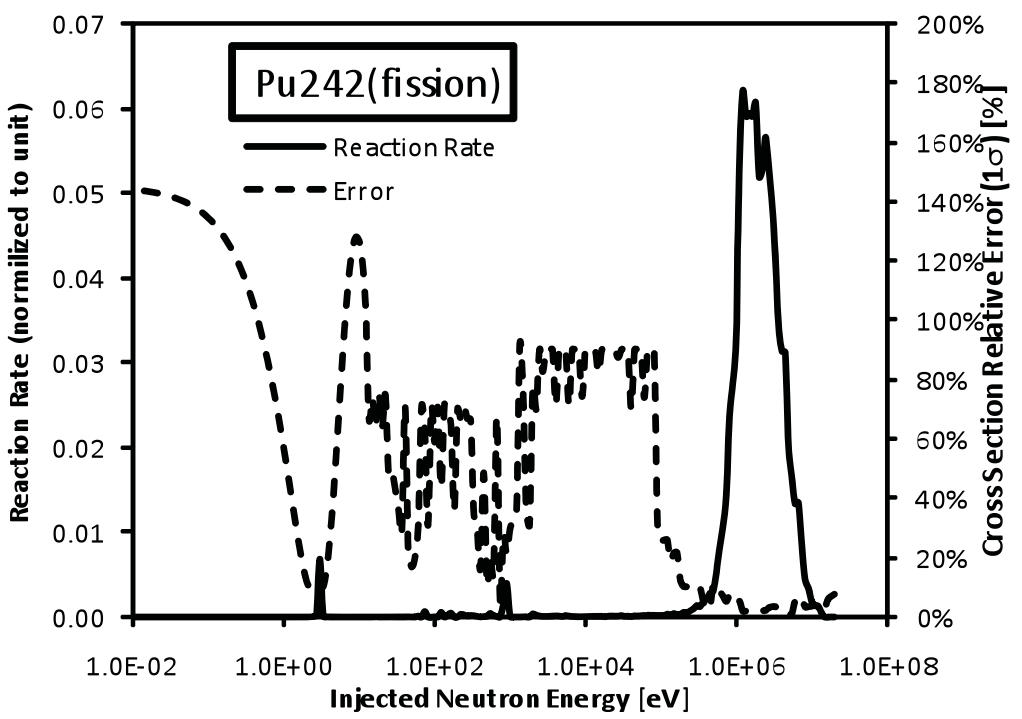

(b) Fission Reaction Rate and Cross Section Error

Figure 15. Pu-242 Energy Dependent Reaction Rate in AFC 2A and Cross-Section Error. 
Americium-241 cross-section error and reaction rate in AFC-2A are shown in Figure 16. Neutron capture cross-section error and reaction rate are shown in Figure 16(a), fission cross-section error and reaction rate are shown in Figure 16(b). The covariance data of Am-241 is derived from both JENDL3.3 and LowFidelity Covariance, with figures and tables in the present report evaluated with JENDL3.3 as representative. In neutron capture reaction, due to the unique neutron spectrum in AFC 2A, almost all the neutron capture reactions occurred with epi-thermal neutrons. The effective one-group capture crosssection error in AFC-2A is calculated as $12 \%$. Neutron fission reaction, due to large fast fission and partial resonance fission, occurred with mostly fast neutron and partly epi-thermal neutrons. The effective one-group fission cross-section error in $\mathrm{AFC}-2 \mathrm{~A}$ is calculated as $6 \%$.

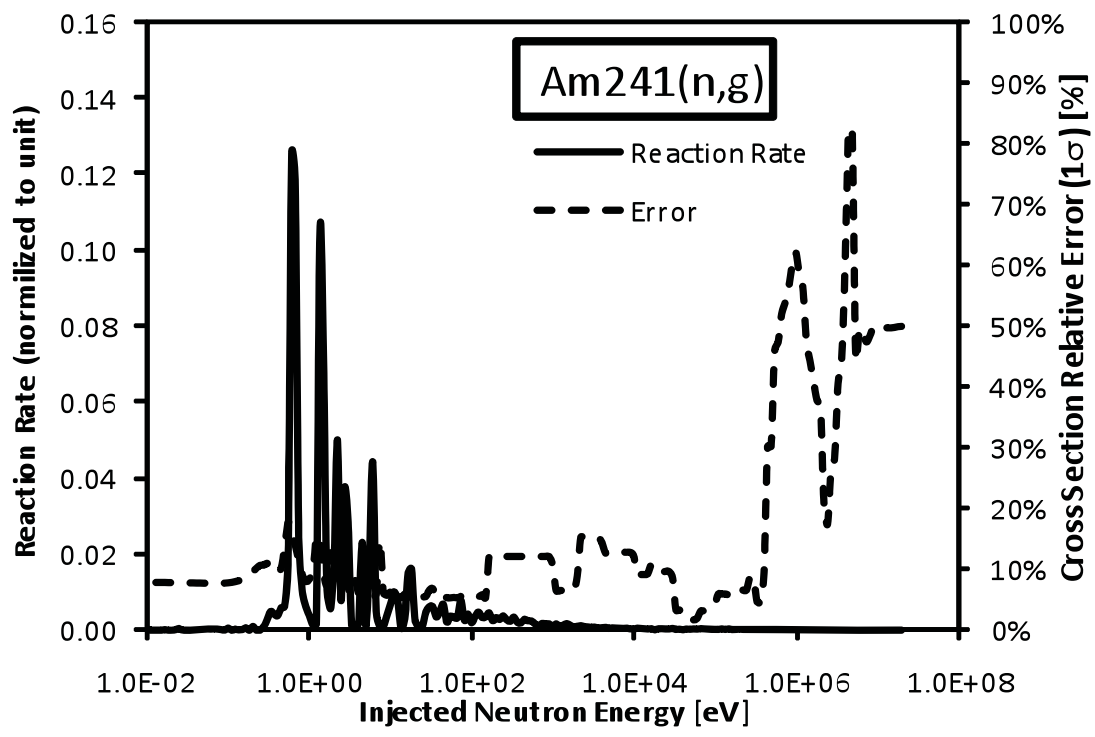

(a) Capture Reaction Rate and Cross Section Error

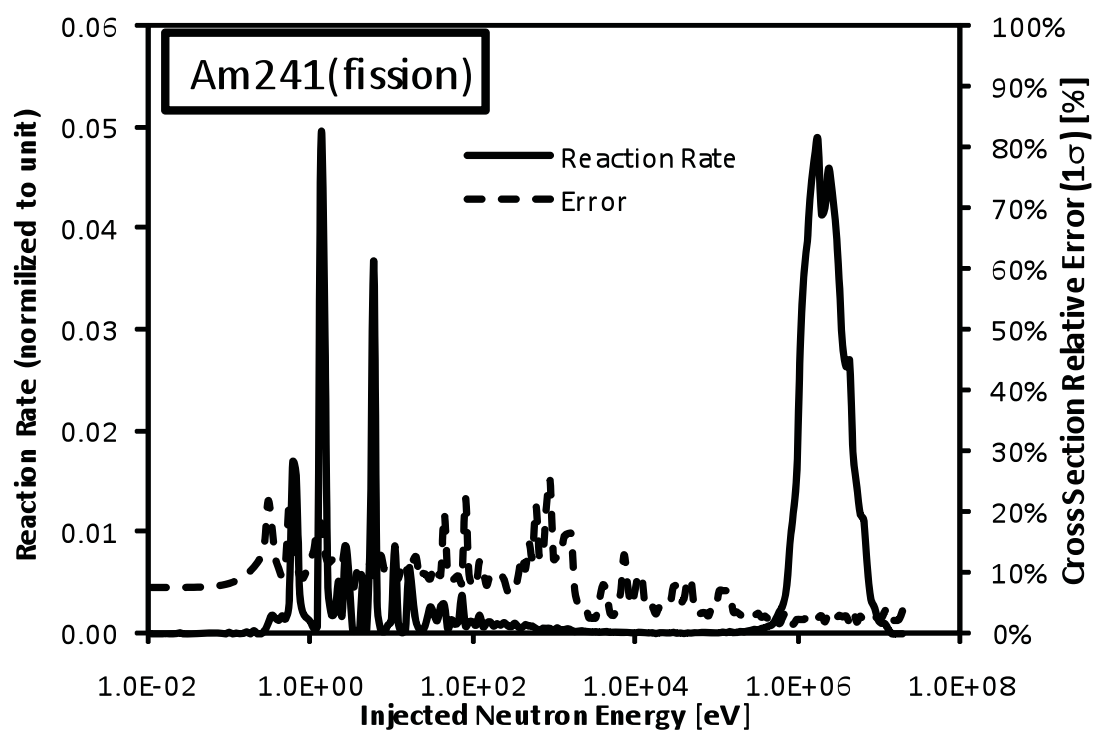

(b) Fission Reaction Rate and Cross Section Error

Figure 16. Am-241 Energy Dependent Reaction Rate in AFC 2A and Cross-Section Error. 
Americium-243 cross-section error and reaction rate in AFC-2A are shown in Figure 17. Neutron capture cross-section error and reaction rate are shown in Figure 17(a), fission cross-section error and reaction rate are shown in Figure 17(b). The covariance data of $\mathrm{Pu}-243$ is derived from both JENDL3.3 and LowFidelity Covariance, and figures and tables in the present report present JENDL3.3 results as representative. In neutron capture reaction, due to the unique neutron spectrum in AFC $2 \mathrm{~A}$, almost all the neutron capture reactions occurred with epi-thermal neutron from one to tens of $\mathrm{eV}$. The effective onegroup capture cross-section error in $\mathrm{AFC}-2 \mathrm{~A}$ is calculated as $7 \%$. In neutron fission reaction, due to large fast fission and partial resonance fission, neutron fission reactions occurred with mostly fast neutron and partly epi-thermal neutrons, the effective one-group fission cross-section error in AFC-2A is calculated as $6 \%$.

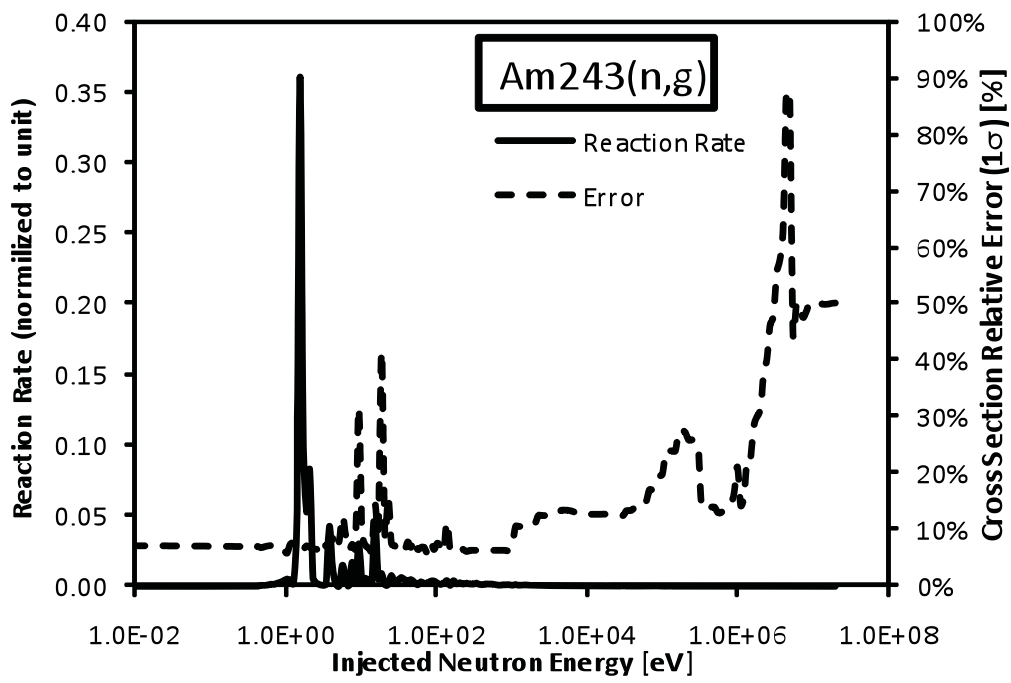

(a) Capture Reaction Rate and Cross Section Error

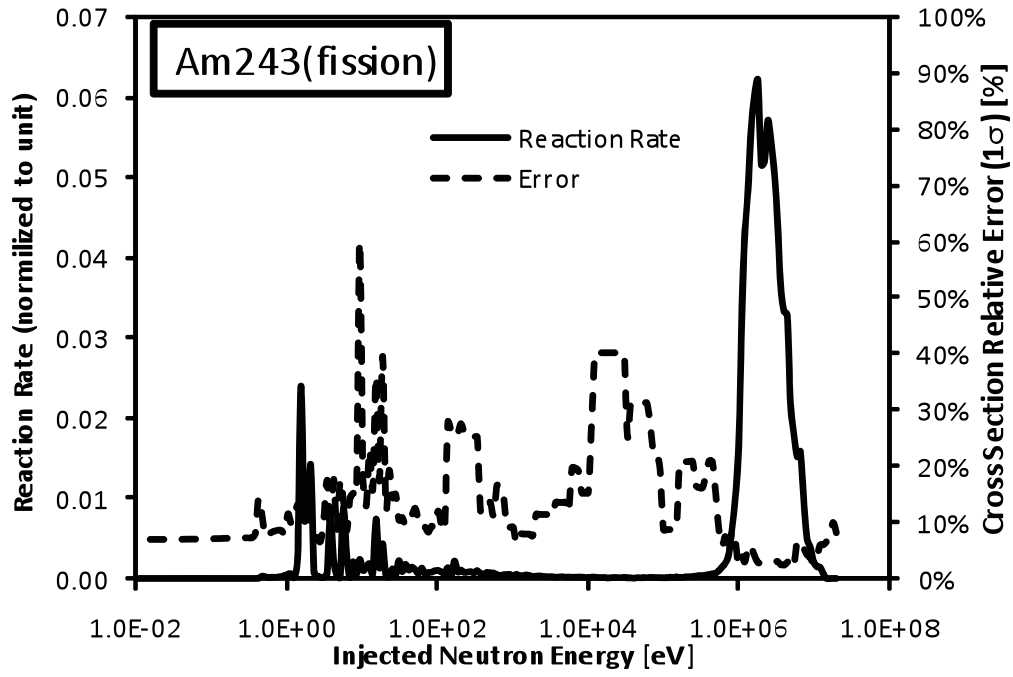

(b) Fission Reaction Rate and Cross Section Error

Figure 17. Am-243 Energy Dependent Reaction Rate in AFC 2A and Cross-Section Error. 
Curium-244 cross-section error and reaction rate in AFC-2A are shown in Figure 18. Neutron capture cross-section error and reaction rate are shown in Figure 18(a), fission cross-section error and reaction rate are shown in Figure 18(b). The covariance data of Cm-244 is derived from both JENDL3.3 and LowFidelity Covariance, and figures and tables in the present report were evaluated with JENDL3.3 and are shown as representative. Neutron capture reaction, due to the unique neutron spectrum in AFC $2 \mathrm{~A}$, almost all occurred with epi-thermal neutrons from one to tens of $\mathrm{eV}$. Due to large cross-section error, the effective one-group capture cross-section error in AFC-2A is calculated as $22 \%$. Neutron fission reaction, due to large fast fission and partial resonance fission, neutron fission reactions occurred with mostly fast neutron and partly epi-thermal neutrons. The effective one-group fission cross-section error in AFC-2A is calculated as $19 \%$.

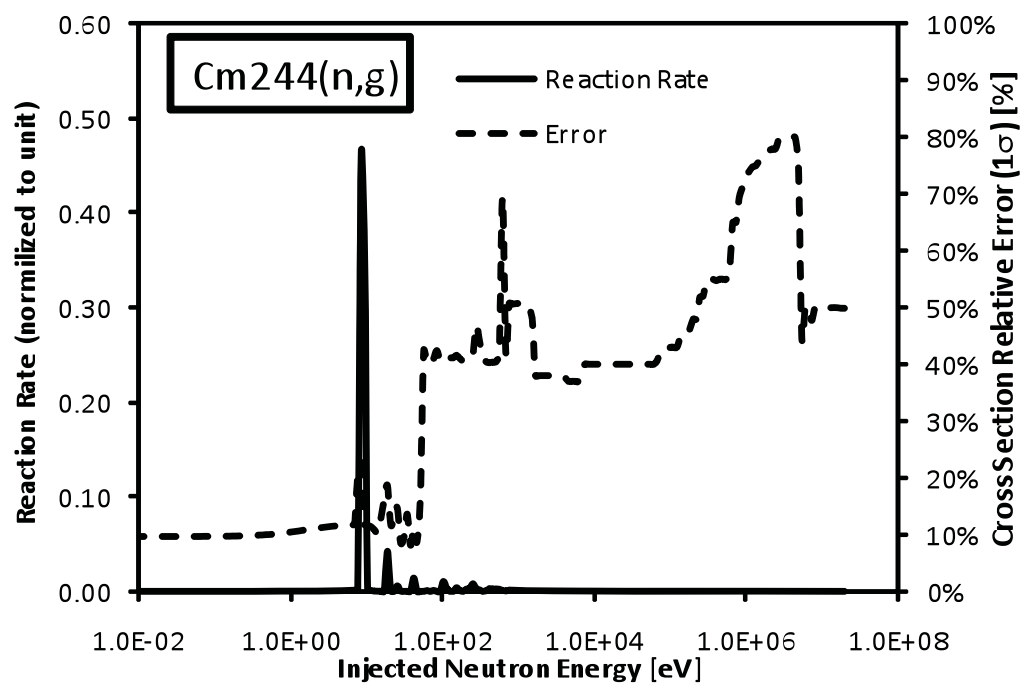

(a) Capture Reaction Rate and Cross Section Error

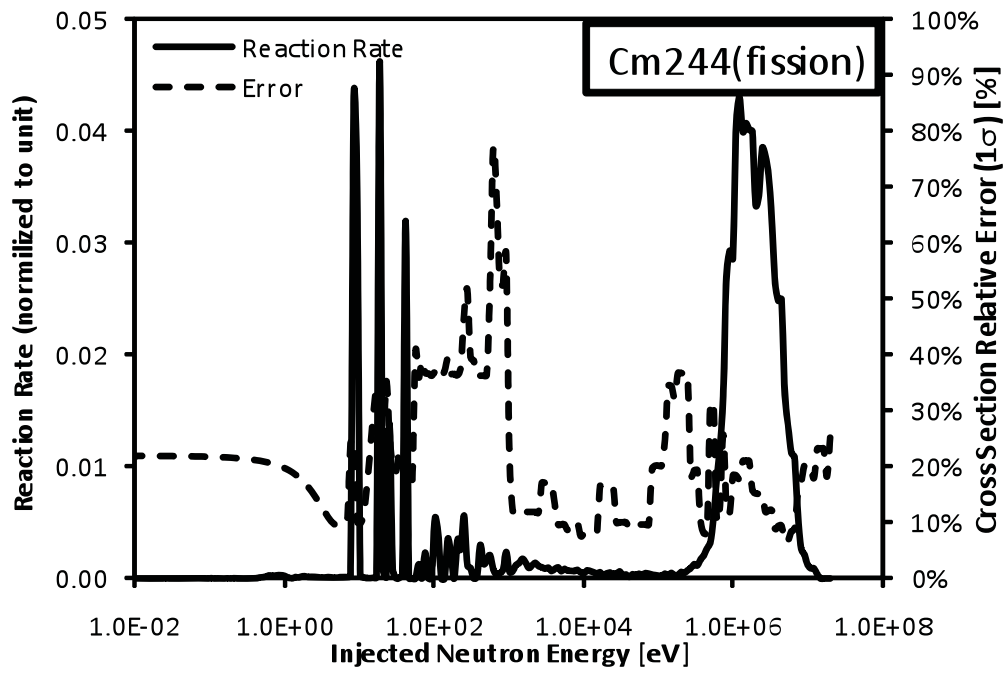

(b) Fission Reaction Rate and Cross Section Error

Figure 18. Cm-244 Energy Dependent Reactions Rate in AFC 2A and Cross-Section Error. 
As a summary of the evaluation of effective cross-section error in AFC-2A, all cross-section errors of all nuclides and reactions are summarized in Table 3.

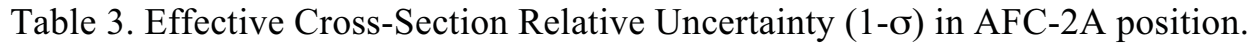

Nuclear Reactions

\begin{tabular}{llll} 
& fission & capture & n.2n \\
\hline U235 & $1 \%$ & $1 \%$ & $10 \%$ \\
U238 & $1 \%$ & $3 \%$ & $3 \%$ \\
NP237 & $3 \%$ & $5 \%$ & \\
PU238 & $18 \%$ & $27 \%$ & \\
PU239 & $6 \%$ & $4 \%$ & $205 \%$ \\
PU240 & $1 \%$ & $2 \%$ & $25 \%$ \\
PU241 & $0.3 \%$ & $0.5 \%$ & $5 \%$ \\
PU242 & $4 \%$ & $4 \%$ & \\
AM241 & $6 \%$ & $12 \%$ & \\
AM243 & $5 \%$ & $7 \%$ & \\
CM244 & $19 \%$ & $22 \%$ & \\
\hline
\end{tabular}

\subsection{Depletion and Buildup Calculation Results and Global Uncertainty}

By using Table 3, global uncertainty analysis of HM depletion and buildup has been performed. As- run depletion and buildup calculations are performed using the MCWO code, and by applying the error data in Table 3. Additional depletion and buildup calculations are also performed to calculate the global uncertainty of each nuclide by using Eq. 5 .

\subsubsection{HM Isotope Density}

Table 4 shows the atomic density of HM in AFC-2A and -2B at the end of irradiation cycle 142B.

Table 4(a) is the atomic density of uranium and neptunium isotopes, Table 4(b) is the atomic density of plutonium isotopes, Table 4(c) is the atomic density of americium isotopes, and Table 4(d) is the atomic density of curium isotopes. As for the reference, initial HM load at the beginning of irradiation of cycle $140 \mathrm{~A}$ is listed in Table 5.

In Table 4(a), since initial uranium enrichment and loading are different in each position and each rodlet as shown in Table 1, the atomic density of uranium isotopes at the end of Cycle 142B is varied. Uranium235 has the same cross-section errors for capture and fission reactions (1\%), and due to massive initial load, the uncertainty of U-235 atomic density at the end of irradiation is quite small, $0.1 \%$. On the other hand, U-236 has no initial load and it is accumulated from the U-235(n,g) reaction, the uncertainty of U236 is mainly affected by that reaction and is around 1\%. The sensitivity of cross-section errors to U-236 atom density uncertainty is shown in Figure 19. Both U-235(n,g) and U-235(n,f) cross-section error are affecting the U-236 atom density uncertainty and the majority is, as expected, U-235(n,g) cross-section error. Sensitivity of U-236 cross-section error is negligibly small because of the massive initial load of U235. Uranium-238 has massive initial load and small cross-section error (1\% U-238(n,f) and 3\% U$238(\mathrm{n}, \mathrm{g}))$, so the uncertainty of U-238 atom density after the irradiation is very low, $0.05 \%$.

Neptunium-237 has rather large resonance capture cross-section at $0.6 \mathrm{eV}$ and $1.7 \mathrm{eV}$, depletion ratio is varied from $10-17 \%$ in $\mathrm{AFC}-2 \mathrm{~A}$ and $-2 \mathrm{~B}$ rodlets, and its effective cross-section error is $5 \%$ in capture and 
$3 \%$ in fission. However, due to the initial load of $\mathrm{Np}-237$, the uncertainty of atomic density is kept less than $1 \%$ (Table 4(a)). The sensitivity of cross-section errors to Np-237 atom density uncertainty is shown in Figure 20. Uranium-235(n,g), U-236(n,g), Np-237(n,g) and Np-237(n,f) cross-section errors are affecting the Np-237 atom density uncertainty and the majority is by Np-237(n,g) cross-section error.

Table 4(b) shows the atomic density of plutonium isotopes. Though major isotopes such as $\mathrm{Pu}-239, \mathrm{Pu}-$ 240 and $\mathrm{Pu}-241$ have small uncertainty of atomic density, the minor isotopes, $\mathrm{Pu}-238$ and $\mathrm{Pu}-242$ have relatively large uncertainty of atomic density. The sensitivity of cross-section errors to Pu- 238 atom density uncertainty is shown in Figure 21. Various factors are affecting the uncertainty of Pu-238 because it is the daughter nuclide of Np-237 and Am-241 transmutation, and especially Am-241(n,g), Np-237(n,g) and $\mathrm{Pu}-238(\mathrm{n}, \mathrm{g})$ errors are the major factors to the uncertainty. The sensitivity of cross-sections errors to $\mathrm{Pu}-242$ atom density uncertainty is shown in Figure 22. Americium-241(n,g), Pu-242(n,g) and Pu$240(\mathrm{n}, \mathrm{g})$ are affecting the uncertainty of $\mathrm{Pu}-242$. As shown in the transmutation chain(Figure 3), there are mainly 2 paths to build up Pu-242, one is the Pu-241(n,g)->Pu-242 and the other is Am-241(n,g)-> Am242(EC) -> Pu-242. Since the Am-241(n,g) cross section and its error are relatively large, Am-241(n,g) errors work as major factors to the uncertainty.

Table 4(c) is the atomic density of americium isotopes. Initial load of Am consisted of pure Am-241. It has a large capture cross section and depletion ratio were observed from 17 to $27 \%$ in AFC-2A, $2 \mathrm{~B}$ rodlets, and the cross section error of the nuclide is also large( $6 \%$ in fission and $12 \%$ in capture reaction). Transmutation of Am-241 affect very much the daughter accumulation and their uncertainty, Am-242m and Am-243 have 13\% uncertainty of their atom density. The sensitivity of cross-sections errors to Am241 atom density uncertainty is shown in Figure 23. Americium-241(n,g) and Am-241(n,f) are affecting the uncertainty of Am-241, and Am-241(n,g) error is the dominant factor of the uncertainty. The sensitivity of cross-sections errors to Am-243 atom density uncertainty is shown in Figure 24Figure 19. As shown in the transmutation chain(Figure 3), there are mainly 2 paths to build up Am-243. One is the $\mathrm{Pu}-242(\mathrm{n}, \mathrm{g})->\mathrm{Am}-243$ and the other is Am-241(n,g)-> Am-242m -> Am-243, and Am-241(n,g), Pu$242(n, g)$ errors are the major factors to the uncertainty.

Table 4(d) is the atomic density of curium isotopes. There is no initial load of $\mathrm{Cm}$, so all $\mathrm{Cm}$ is accumulated through the transmutation of Am. Cm-244 has shortage of measurement and large cross section errors (19\% in fission and $22 \%$ in capture). Since the same error as $\mathrm{Cm}-244$ is applied to other $\mathrm{Cm}$ isotopes in the present work, the uncertainty of $\mathrm{Cm}$ isotopes is very large. The sensitivity of crosssections errors to Cm-242 atom density uncertainty is shown in Figure 25. Americium-241(n,g) and $\mathrm{Cm}-242(\mathrm{n}, \mathrm{g})$ are affecting the uncertainty of Cm-242 atom density, and Am-241(n,g) error is the dominant factor of the uncertainty. The sensitivity of cross-sections errors to $\mathrm{Cm}-244$ atom density uncertainty is shown in Figure 26. As shown in the transmutation chain(Figure 3), there are mainly 2 paths to build up Cm-244. One is the Am-243(n,g)->Cm-244 and the other is Cm-243(n,g)->Cm-244, and $\mathrm{Pu}-242(\mathrm{n}, \mathrm{g})$ and $\mathrm{Am}-241(\mathrm{n}, \mathrm{g})$ errors are the major factors to the uncertainty.

Time dependent HM depletion with uncertainty is shown in Figure 27, for AFC-2A rodlet 3. Nuclides relating with MA transmutation are selected in the figure to confirm the transport of errors by nuclides and by irradiation time. 

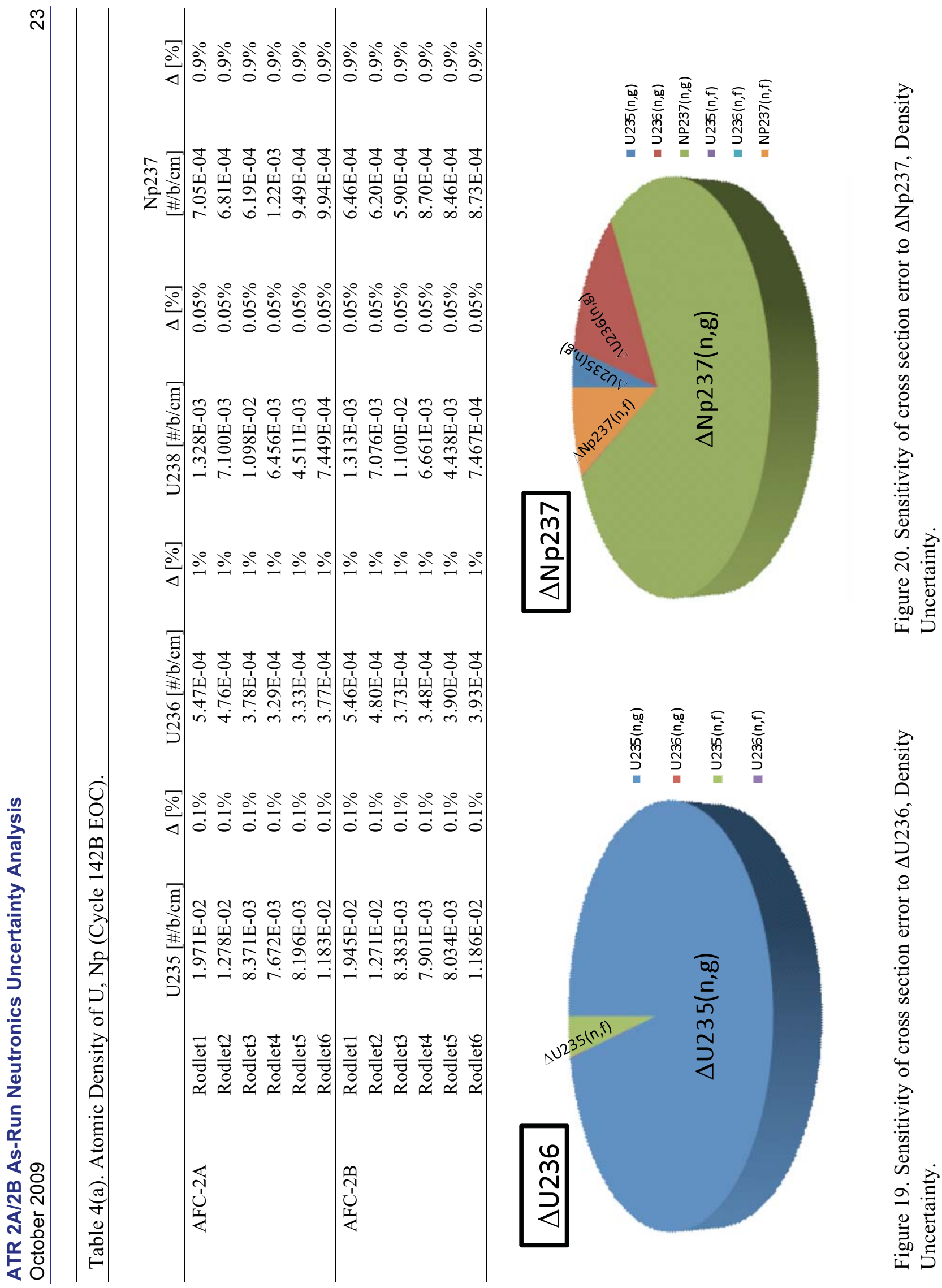

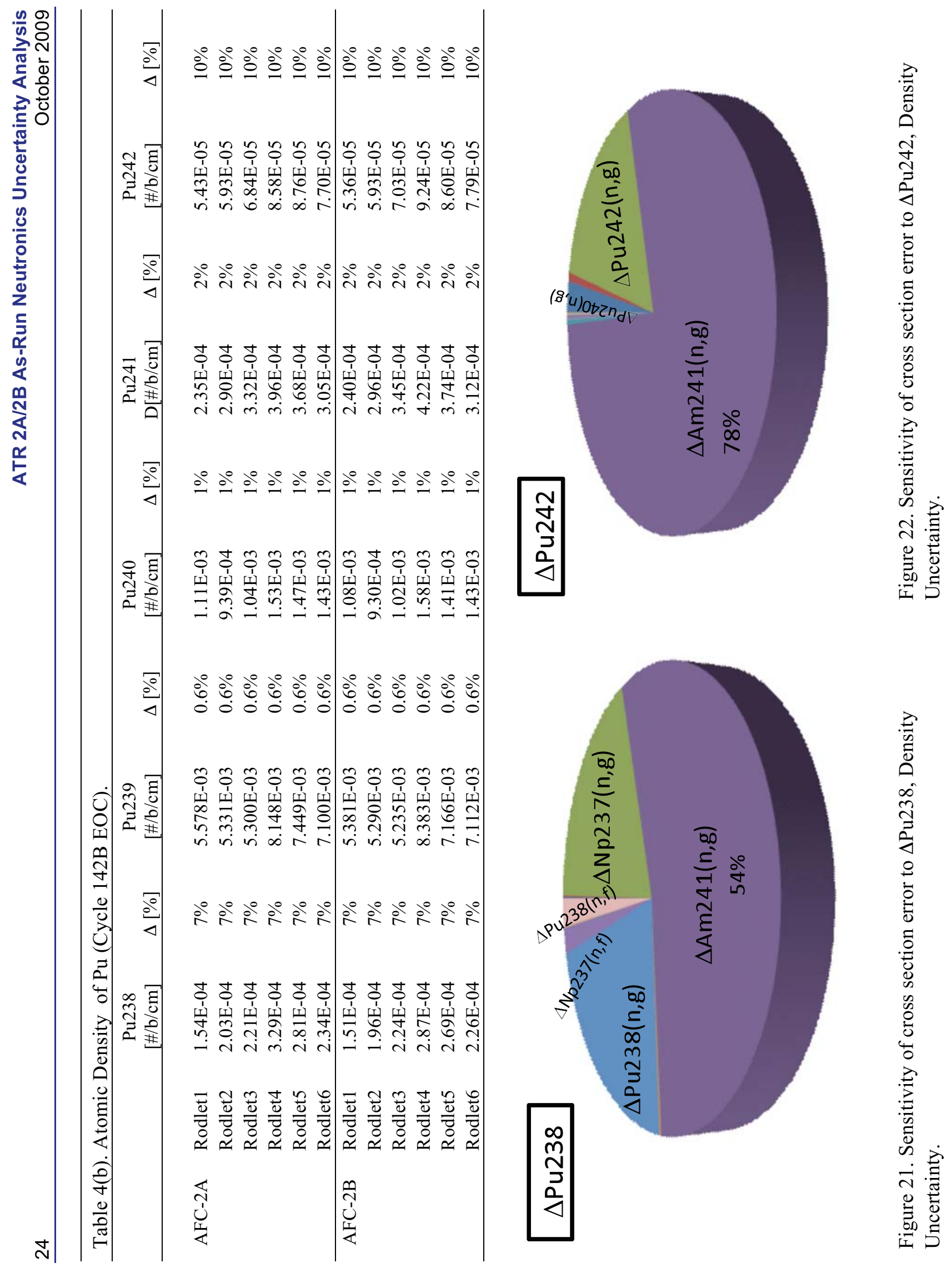


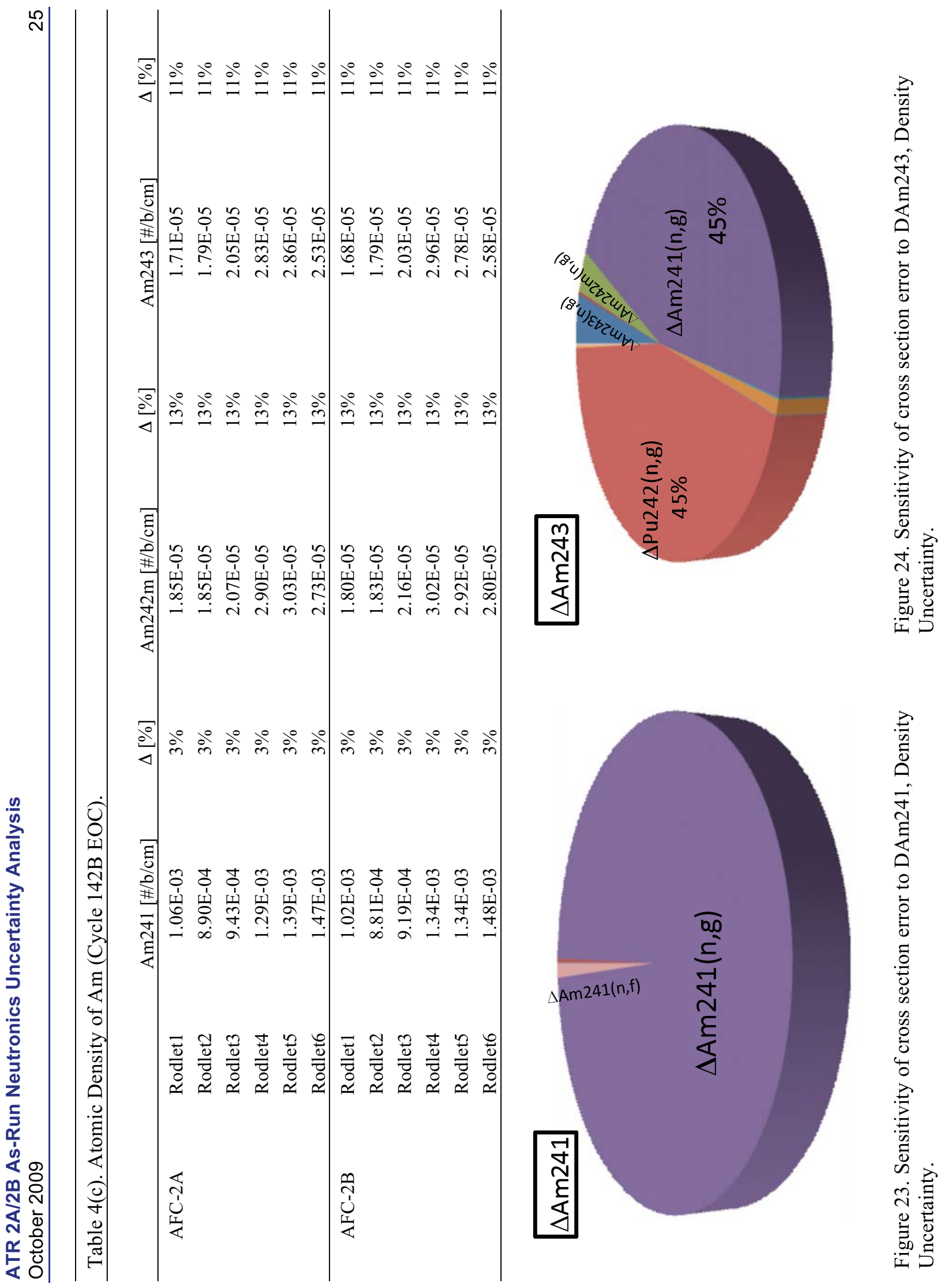



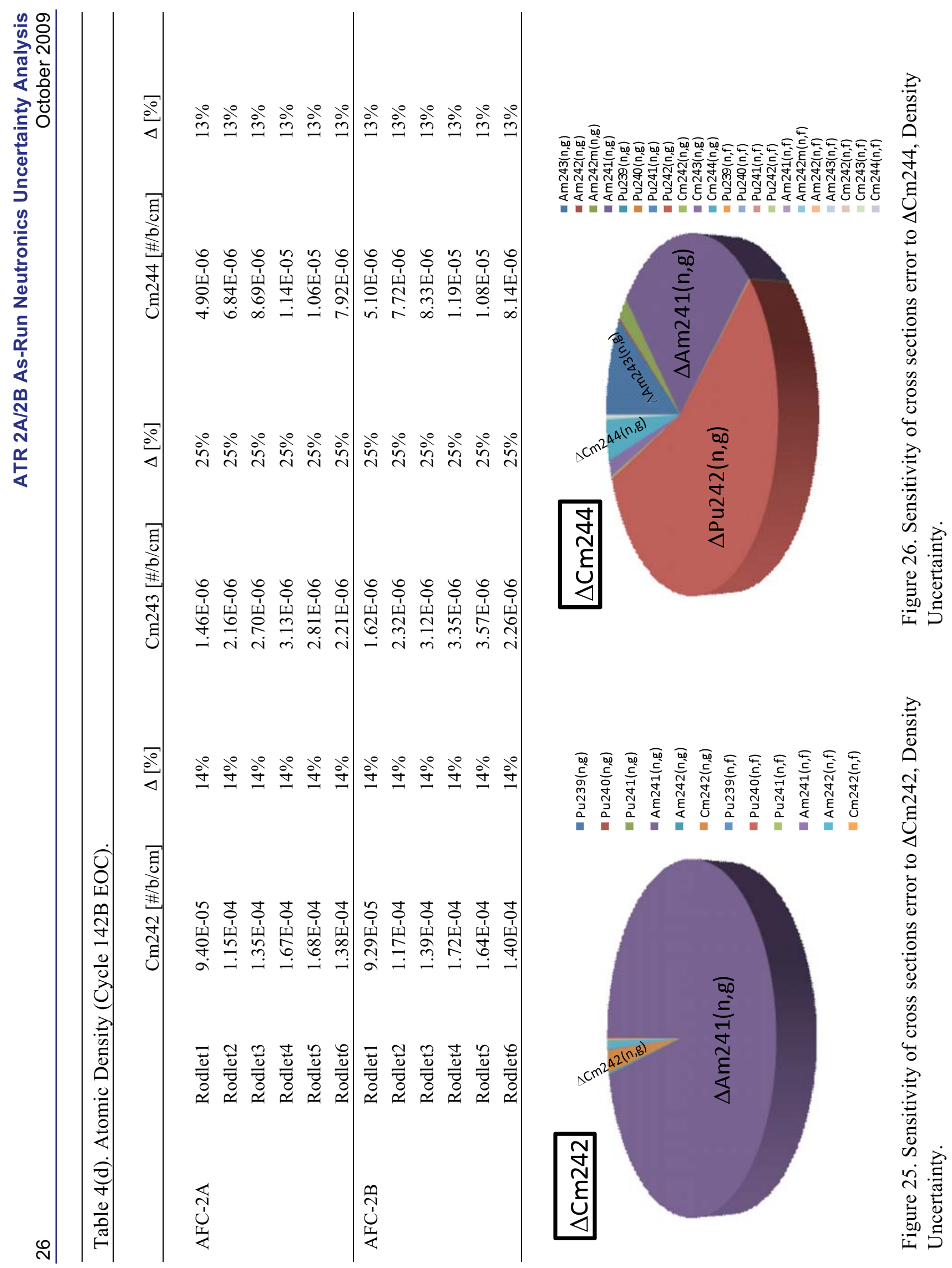

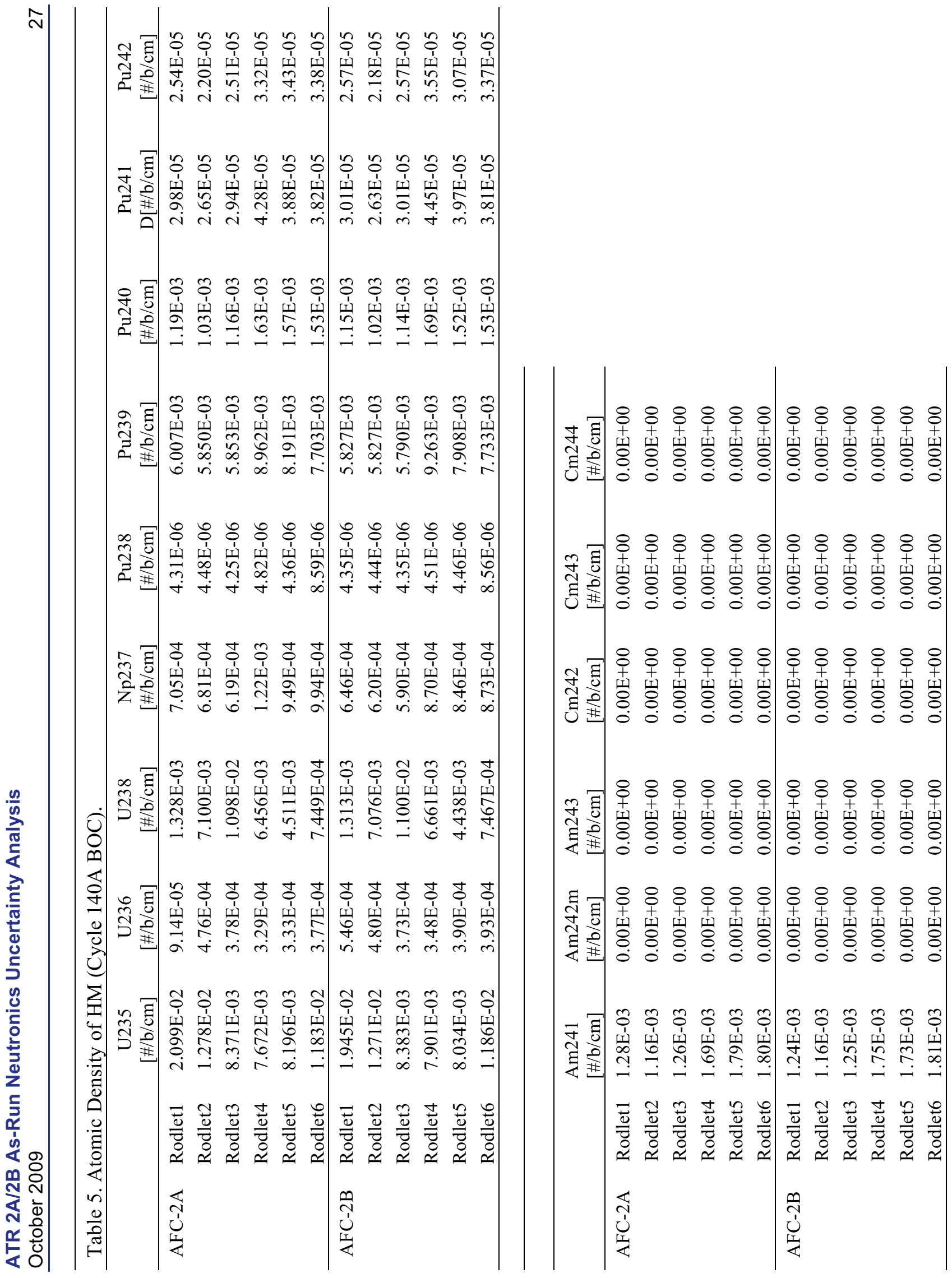


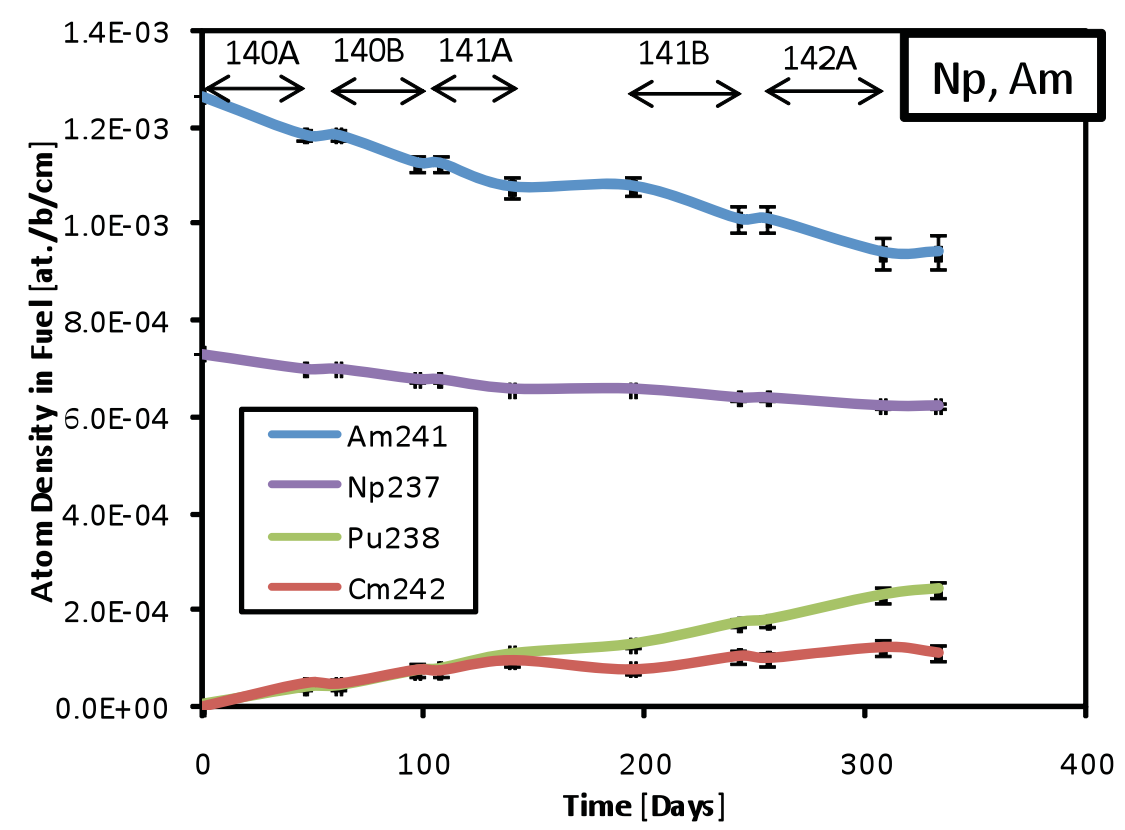

(a) Np-237, Am-241 transmutation and their by-products

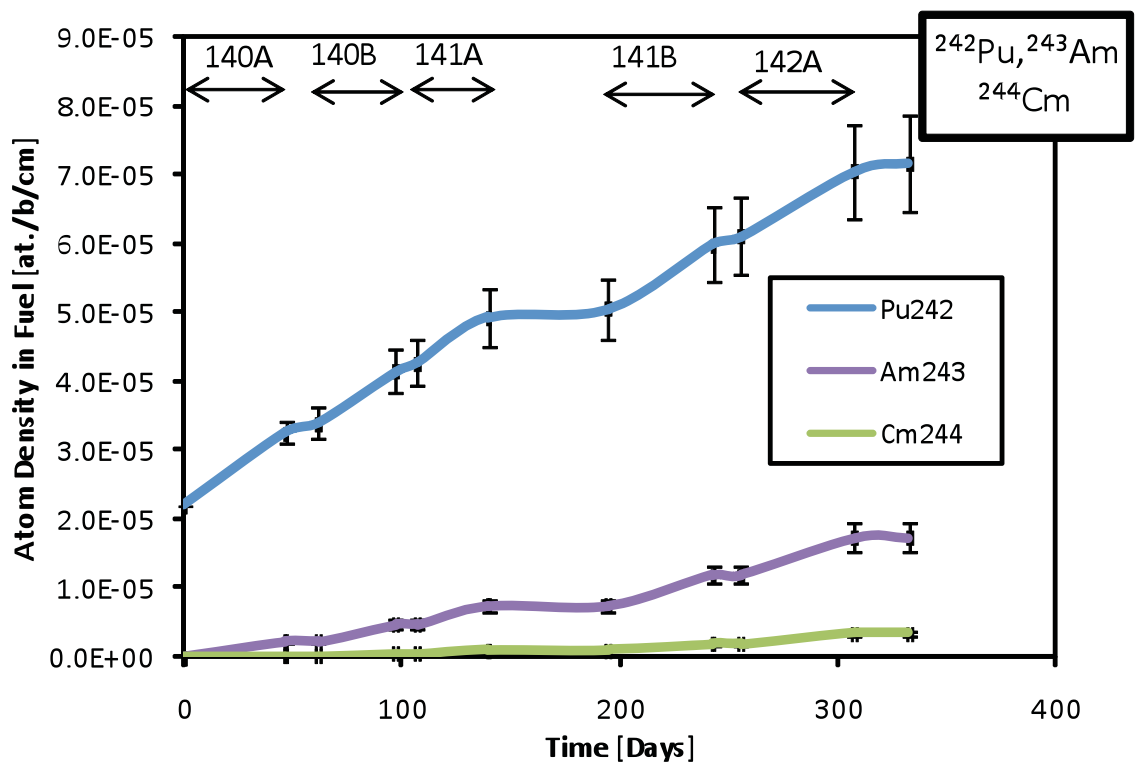

(b) $\mathrm{Pu}-242, \mathrm{Am}-243$ transmutation and their by-products

Figure 27. Time dependent HM depletion and buildup (AFC-2A rodlet 3). 


\subsubsection{HM elemental and isotope composition}

Based on the nuclides density provided in previous section, elemental density (Table 6), elemental composition (Table 7) and isotope composition (Table 8) are calculated with uncertainty. Sensitivity of elemental density and composition uncertainty is shown in Figure 28 . We can visually evaluate the importance of each cross-section error to the final composition.

Uncertainty of the density of element A consists of isotopes A1, A2, A3 .. is described as following,

$$
\Delta N_{A}=\sqrt{\Delta N_{A 1}^{2}+\Delta N_{A 2}^{2}+\Delta N_{A 3}^{2} \cdots}
$$

Uncertainty of the elemental composition consists of elements A, B, C ... is described as following,

$$
\begin{aligned}
R_{A} & \equiv \frac{N_{A}}{N_{A}+N_{B}+N_{C}+\ldots} \\
\Delta R_{A} & =\sqrt{\left(\frac{\partial R_{A}}{\partial N_{A}} \Delta N_{A}\right)^{2}+\left(\frac{\partial R_{A}}{\partial N_{B}} \Delta N_{B}\right)^{2}+\left(\frac{\partial R_{A}}{\partial N_{c}} \Delta N_{c}\right)^{2}+\ldots} \\
& =\sqrt{\left(\frac{N_{B}+N_{c}+\ldots}{\left(N_{A}+N_{B}+N_{c}+\ldots\right)^{2}} \Delta N_{A}\right)^{2}+\left(\frac{-N_{A}}{\left(N_{A}+N_{B}+N_{c}+\ldots\right)^{2}} \Delta N_{B}\right)^{2}+\left(\frac{-N_{A}}{\left(N_{A}+N_{B}+N_{c}+\ldots\right)^{2}} \Delta N_{c}\right)^{2}+\ldots}
\end{aligned}
$$

Uncertainty of the isotopic composition of element A consists of isotopes A1, A2, A3 ... is described as following,

$$
\begin{aligned}
I_{A 1} & \equiv \frac{N_{A 1}}{N_{A 1}+N_{A 2}+N_{A 3}+\ldots} \\
\Delta I_{A 1} & =\sqrt{\left(\frac{\partial I_{A 1}}{\partial N_{A 1}} \Delta N_{A 1}\right)^{2}+\left(\frac{\partial I_{A 1}}{\partial N_{A 2}} \Delta N_{A 2}\right)^{2}+\left(\frac{\partial I_{A 1}}{\partial N_{A 3}} \Delta N_{A 3}\right)^{2}+\ldots} \\
& =\sqrt{\left(\frac{N_{A 2}+N_{A 3}+\ldots}{N_{A}{ }^{2}} \Delta N_{A 1}\right)^{2}+\left(\frac{-N_{A 1}}{N_{A}{ }^{2}} \Delta N_{A 2}\right)^{2}+\left(\frac{-N_{A 1}}{N_{A}{ }^{2}} \Delta N_{A 3}\right)^{2}+\ldots}
\end{aligned}
$$




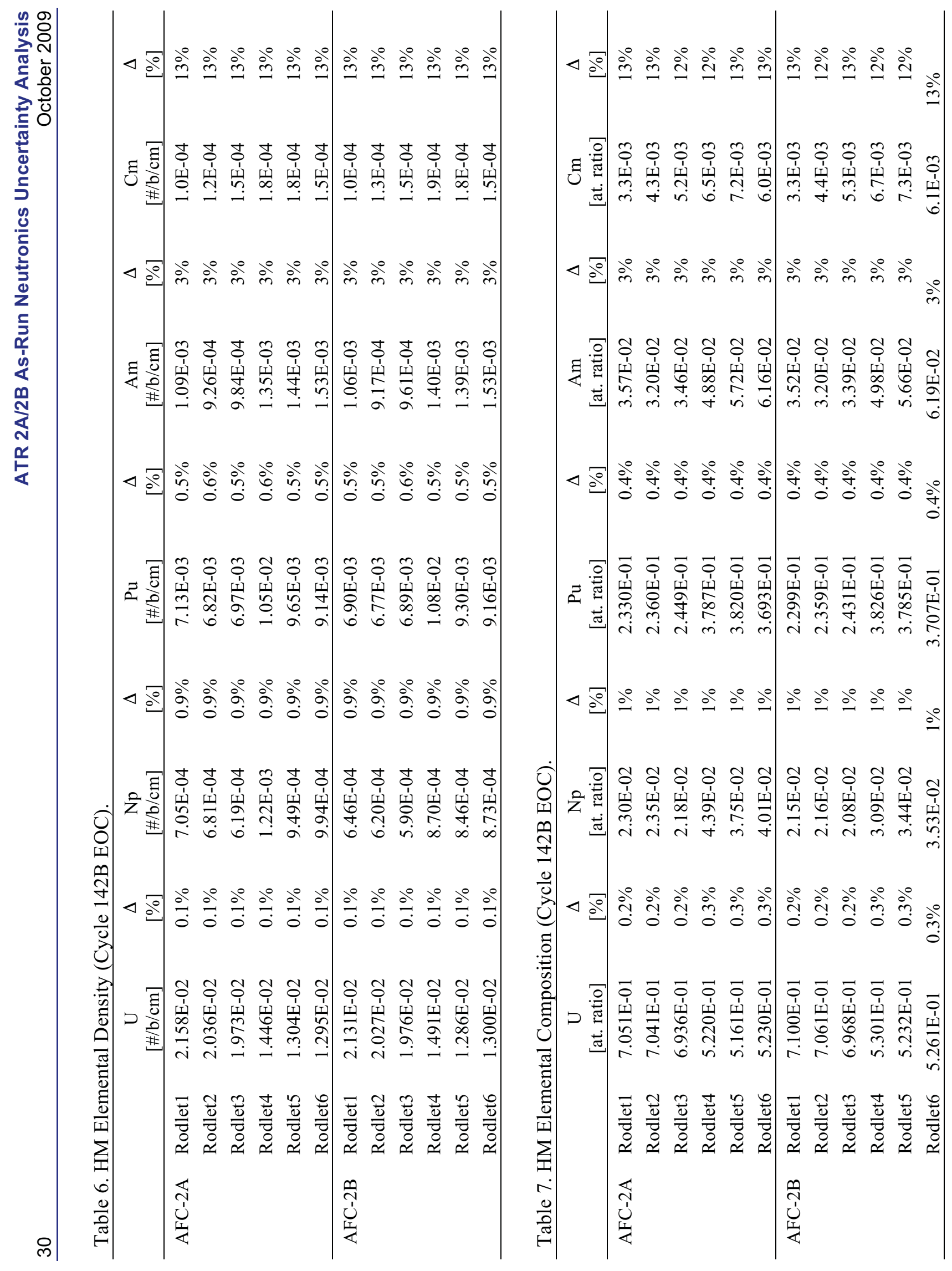



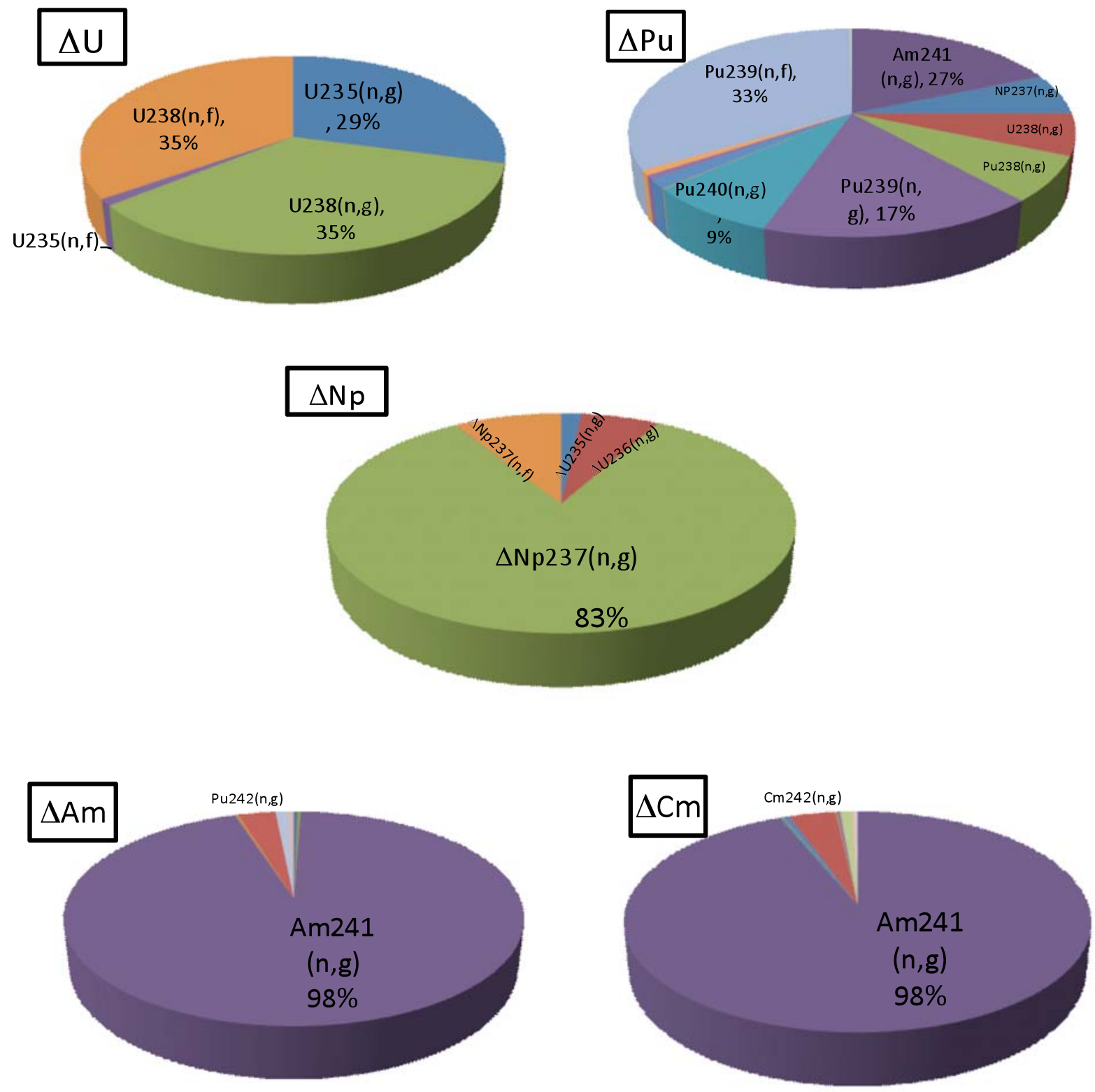

Figure 28. Sensitivity of cross section error to Element Composition Uncertainty (AFC 2A Rodlet 3). 


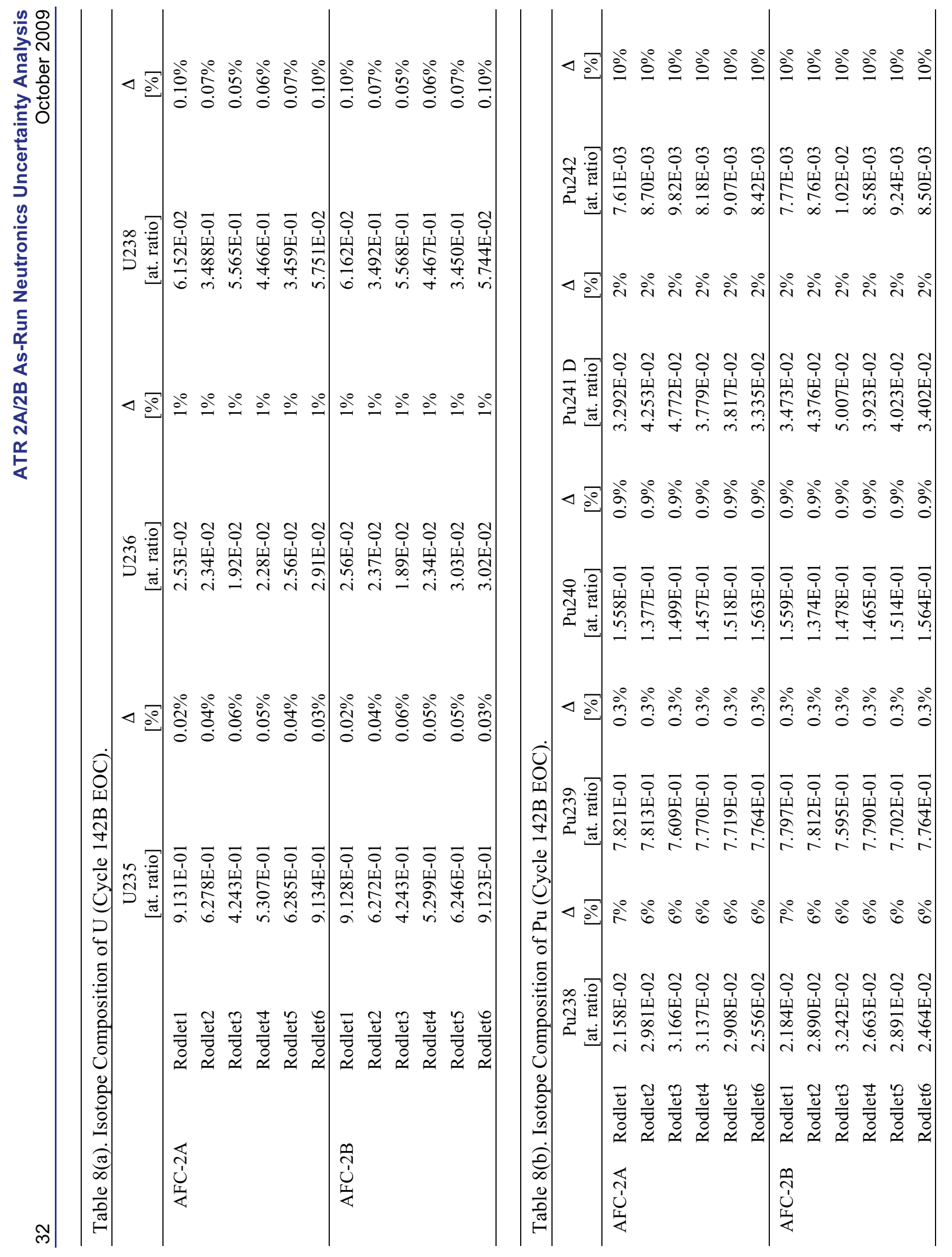




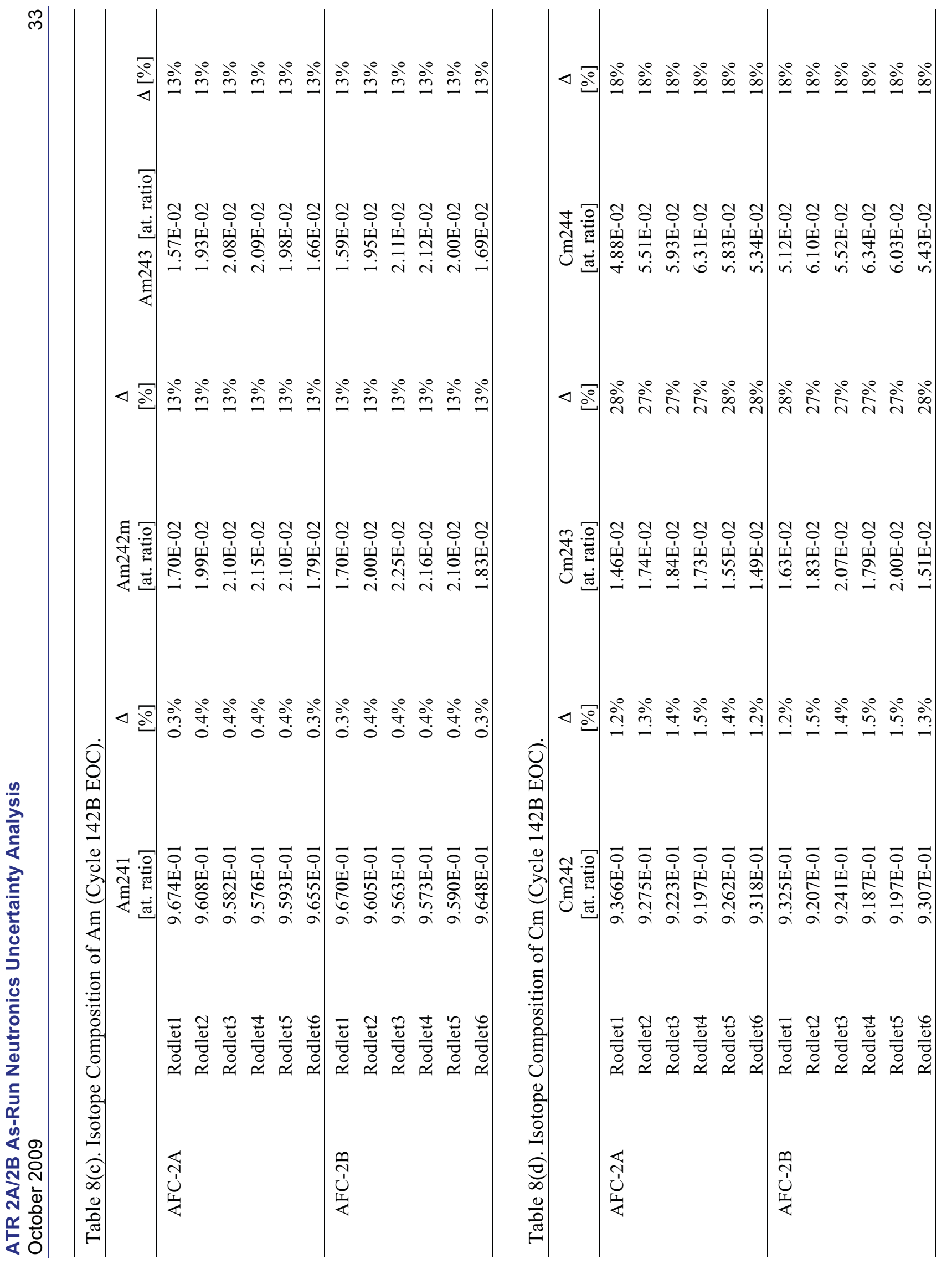




\section{Burnup and its uncertainty}

Based on the nuclides density provided in previous section, burnup (Table 9) is calculated with uncertainty. Sensitivity of cross section error to burnup uncertainty is shown in Figure 29. We can visually evaluate the importance of each cross-section error to burnup.

Uncertainty of Burnup is described as following,

$$
\begin{aligned}
& B U\left[F I M A=\frac{N_{H M_{B O C}}-N_{H M_{E O C}}}{N_{H M_{B O C}}}\right. \\
& \Delta B U=\sqrt{\left(\frac{\partial B U}{\partial N_{H M_{E O C}}} \Delta N_{H M_{E O C}}\right)^{2}}=\left|\frac{\Delta N_{H M_{E O C}}}{N_{H M_{E O C}}}\right|
\end{aligned}
$$

In Table 9, burnups at the end of cycle 142B are listed. Burnup values of each fuel pins are varied from $3.6 \%$ to $4.6 \%$, because of the difference in neutron fluence and fissile nuclide component. As for the uncertainty of burnup in each fuel pin, it is not simply proportional to the burnup. For example, though rodlet1 has much smaller burnup than others, the uncertainty is much larger than rodlet 2 and 3. Comparing rodlet 1 with rodlet3, fuel composition is the same except the enrichment of uranium, $93 \% \mathrm{EU}$ in rodlet 1 and $55 \%$ in rodlet 3 . Since U-235 cross section, especially capture cross section error has big sensitivity to U-236 and Np-237 accumulation, this difference affects the uncertainty of burnup in each fuel pin. As shown in Figure 29(a) and Figure 29(b), the sensitivity of cross section error to burnup uncertainty in rodlet 1 and rodlet 3 can be visually confirmed and major difference is caused by the difference of $\mathrm{U}-235(\mathrm{n}, \mathrm{g})$ error. Comparing rodlet1 with rodlet6, composition of $\mathrm{Pu}, \mathrm{Am}, \mathrm{Np}$ and the enrichment of $U$ is increased in rodlet 6 . As shown in Figure 29(a) and Figure 29(c), the sensitivity of cross section error to burnup uncertainty in rodlet 1 and rodlet6 can be visually confirmed and the major difference is caused by the increasing Am-241(n,g) error.

\begin{tabular}{cccc}
\hline Table 9. BURNUP (Cycle 142B EOC). & & \\
\hline & & FIMA & $\Delta$ \\
[DHM/HMin] & {$[\%]$} \\
\hline AFC-2A & Rodlet1 & $3.625 \mathrm{E}-02$ & $5 \%$ \\
& Rodlet2 & $4.227 \mathrm{E}-02$ & $4 \%$ \\
& Rodlet3 & $3.919 \mathrm{E}-02$ & $5 \%$ \\
& Rodlet4 & $4.341 \mathrm{E}-02$ & $7 \%$ \\
& Rodlet5 & $4.405 \mathrm{E}-02$ & $7 \%$ \\
& Rodlet6 & $4.112 \mathrm{E}-02$ & $7 \%$ \\
\hline AFC-2B & Rodlet1 & $3.846 \mathrm{E}-02$ & $5 \%$ \\
& Rodlet2 & $4.299 \mathrm{E}-02$ & $4 \%$ \\
& Rodlet3 & $4.048 \mathrm{E}-02$ & $5 \%$ \\
& Rodlet4 & $4.539 \mathrm{E}-02$ & $6 \%$ \\
& Rodlet5 & $4.604 \mathrm{E}-02$ & $7 \%$ \\
\hline
\end{tabular}




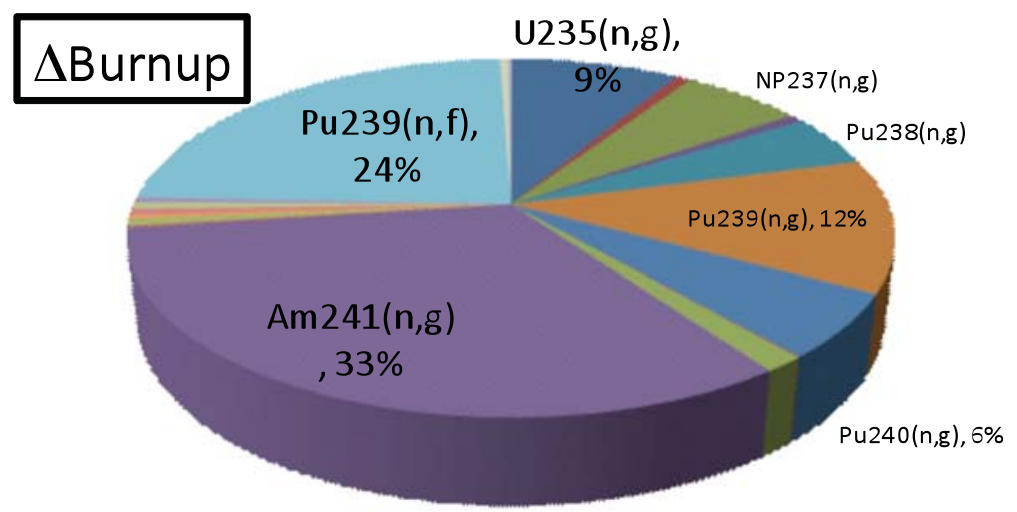

(a) AFC-2A rodlet 1 (U-20Pu-3Am-2Np-15Zr, 93\%EU)

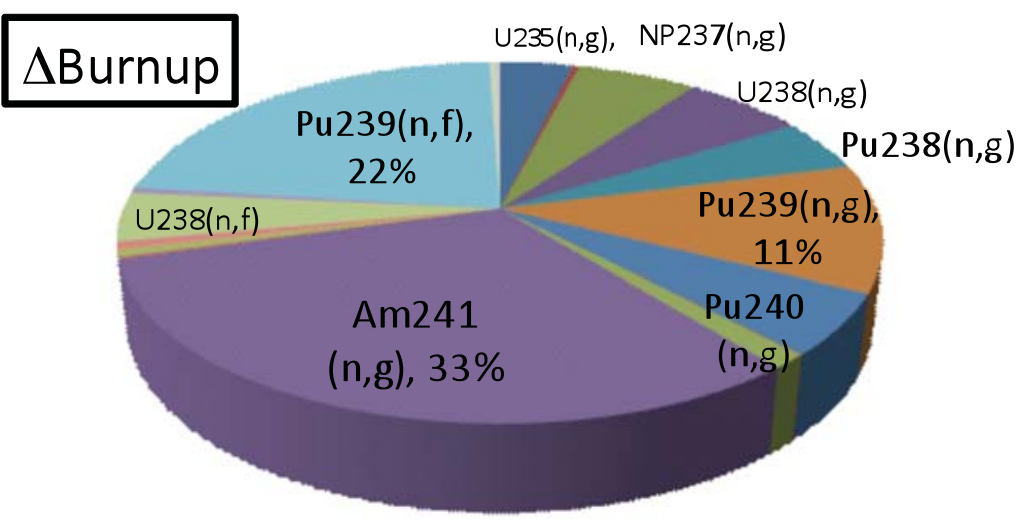

(b) AFC-2A rodlet 3 (U-20Pu-3Am-2Np-15Zr-1.0RE, 55\%EU)

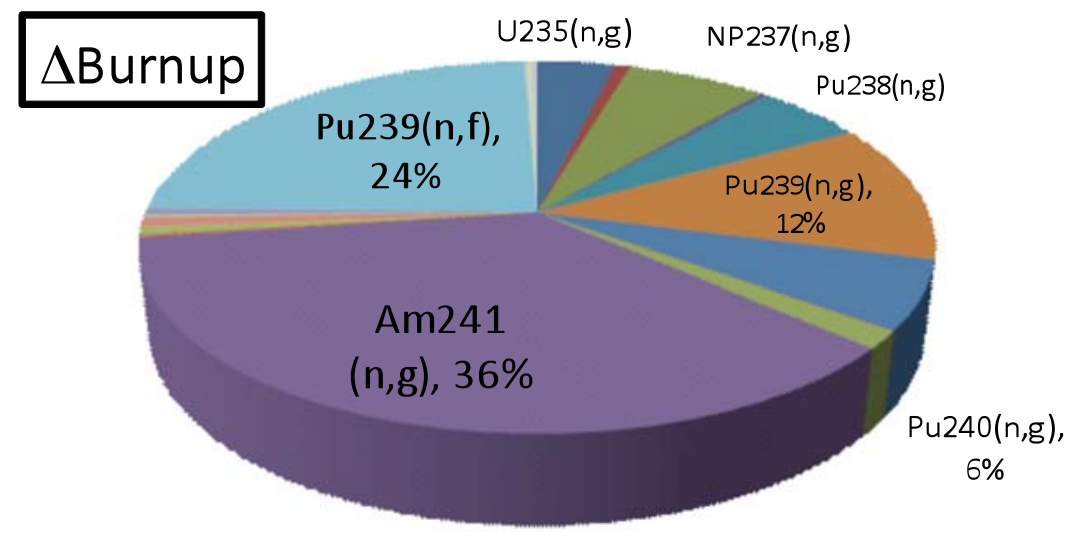

(c) AFC-2A rodlet $6(\mathrm{U}-30 \mathrm{Pu}-5 \mathrm{Am}-3 \mathrm{~Np}-1.0 \mathrm{RE}-15 \mathrm{Zr}, 93 \% \mathrm{EU})$

Figure 29. Sensitivity of Cross-section Error to Burnup Uncertainty. 


\section{CONCLUSION}

Uncertainty analysis relating to calculations for AFC-2A and AFC-2B has been performed. The methodology to treat the nuclear cross section covariance and global uncertainty in depletion and buildup calculations was established by addition to current MCWO code system with interface of ORIGEN Library. This uncertainty analysis methodology was applied for AFC-2A and AFC-2B fuel pin irradiation, and uncertainties of HM density, elemental composition, isotope composition and burnup are calculated. As a result, the sensitivity of cross section errors are evaluated coupling with original as-run calculations by MCWO, and the importance of cross section error to the final output is evaluated by sensitivity analysis. This methodology and results can provide productive information to PIE experimentalists to discuss the discrepancy between calculations and experiments in AFCI program. And also, the uncertainty information can be utilized by the safety evaluation, validation of code and nuclear data, and their improvement.

\section{ACKNOWLEDGEMENT}

To perform the present work, we got lots of productive comment from Fuel and PIE engineers, Dr. Steve Hayes, Mr. Jon Carmack, Dr. Bruce Hilton and Dr. Irina Glagolenko. For processing the error data of Low-Fidelity Covariance by ERRORJ, I achieved big help from Dr. Go Chiba, JAEA, and Scientists in Brookhaven National Laboratory. Special Thanks to Ms. Misti Lillo for her support of calculation environment and Mr. Richard G. Ambrosek for his technical proof-read. We would like to express our great gratitude to them.

\section{REFERENCES}

1. Evaluated Nuclear Data File, http://www.nndc.bnl.gov/exfor/endf00.jsp

2. Japanese Evaluated Nuclear Data Library, http://wwwndc.jaea.go.jp/jendl/jendl.html

3. http://www.nea.fr/html/dbdata/projects/jeffdoc.html

4. http://www.talys.eu/tendl-2008/

5. R.C. Little et al., "Low-Fidelity Covariance Project", Nuclear Data Sheets 109, 2828-2833 (2008). Data can be downloaded from http://www.nndc.bnl.gov/lowfi/

6. H. J. MacLean, D. J. Utterbeck, G. S. Chang, M, A. Lillo, "Advanced Fuel Cycle Initiative AFC-1G, AFC-1H, AFC-2A, and AFC-2B End of FY-08 Irradiation Report," INL/EXT-08 14829 (2008).

7. S. L. Hayes, "Irradiation of Metalic Fuel with Rare Earth Additions for Actinide Transmutation in the Advanced Test Reactor, Experiment Description for AFC-2A and AFC-2B," INL/EXT-06 11707 (2007).

8. S. L. Hayes, D. J. Utterbeck, "Project Description Advanced Fuel Cycle Initiative AFC-2A and AFC-2B Experiments," INL/EXT-07-12388 (2007)

9. INL/EXT-07-12388

10. INL/EXT-07-13021

11. ORNL, "ORIGEN 2.2 Isotope Generation and Depletion Code Matrix Exponential Method," CCC-371, RSICC(2002).

12. G Chang, "MCWO: Monte Carlo , depletion methodology to Coupling MCNP5 with ORIGEN2," INL 
13. G. Chiba, "ERRORJ : A Code to Process Neutron-nuclide Reaction Cross Section Covariance, Version 2.3," JAEA-Data/Code 2007-007 (2007).

14. Kosako K. and Yamano N., 'Preparation of a Covariance Processing System for the Evaluated Nuclear Data File, JENDL, (III),' JNC TJ 9440 99-003, Japan Nuclear Cycle Development Institute (1999) [in Japanese].

15. MacFarlane R.E. and Muir D.W., 'The NJOY Nuclear Data Processing System version 91,' LA12740-M, Los Alamos National Laboratory (1994).

16. Wiarda D., Dunn M.E., el al., 'New Capability for Processing Covariance Data in Resonance Region,' Proc. of Int. Conf. of Reactor Physics (PHYSOR-2006), (CD-ROM) (2006).

17. D. H. Kim, el al., 'Sensitivity and uncertainty analysis on the criticality by an ERRORJ/SUSD3D with JENDL-3.3 covariance data,' Proc. International Conference on Nuclear Data for Science and Technology (2007). 


\section{Appendix A}

\section{Results of Isolated EFT Model with Boundary Neutron Source $(\Delta \phi=5-10 \%)$}



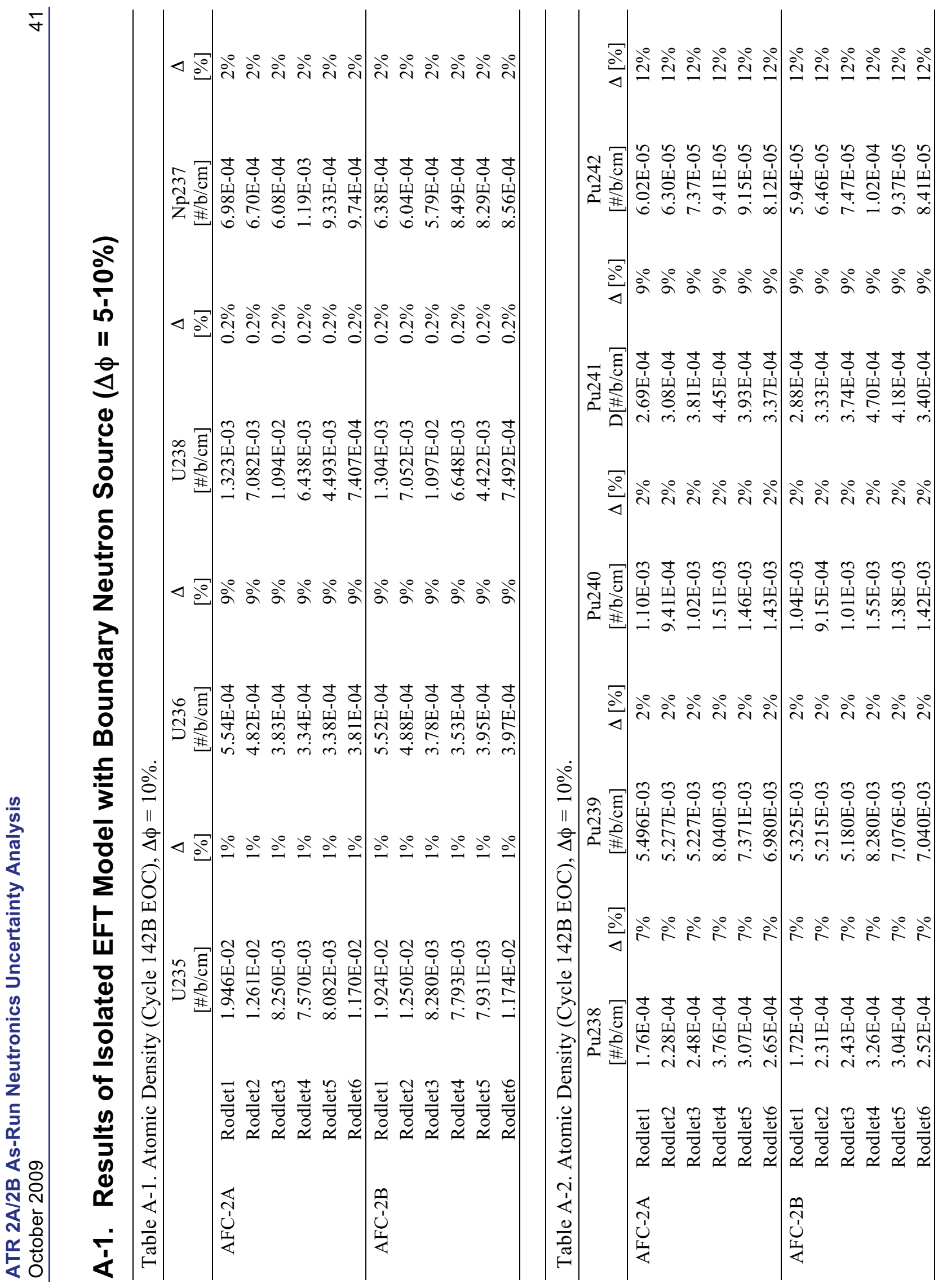


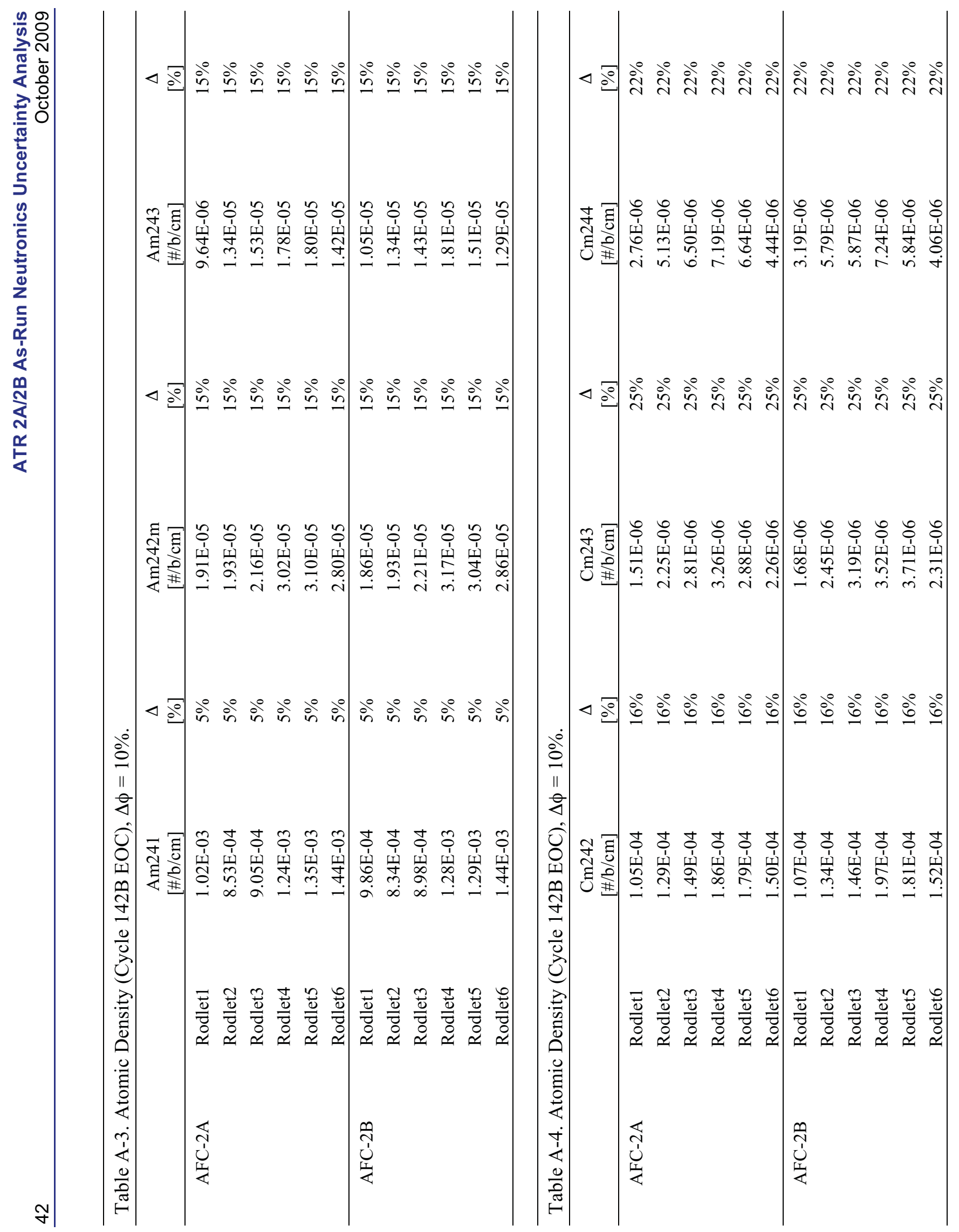




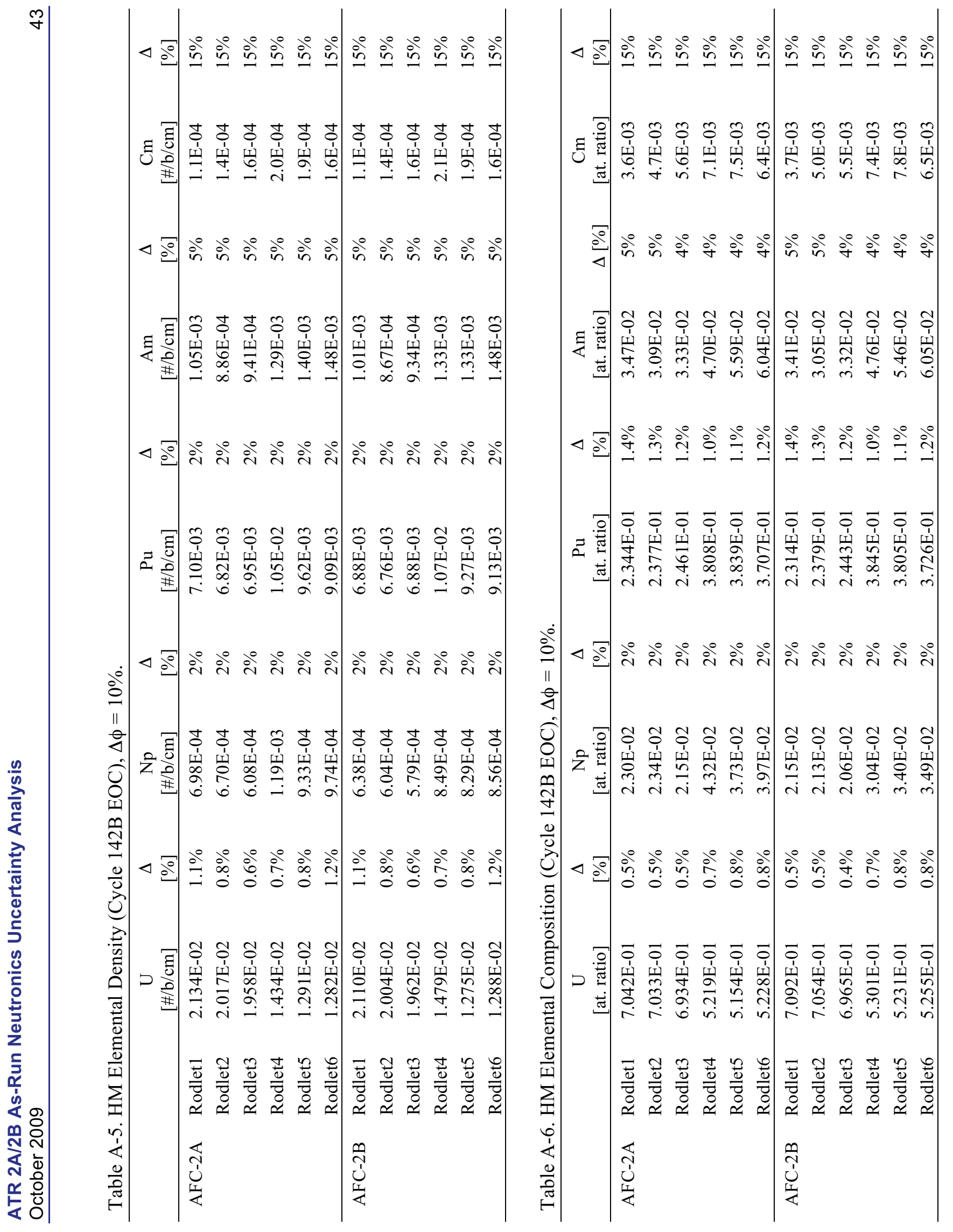




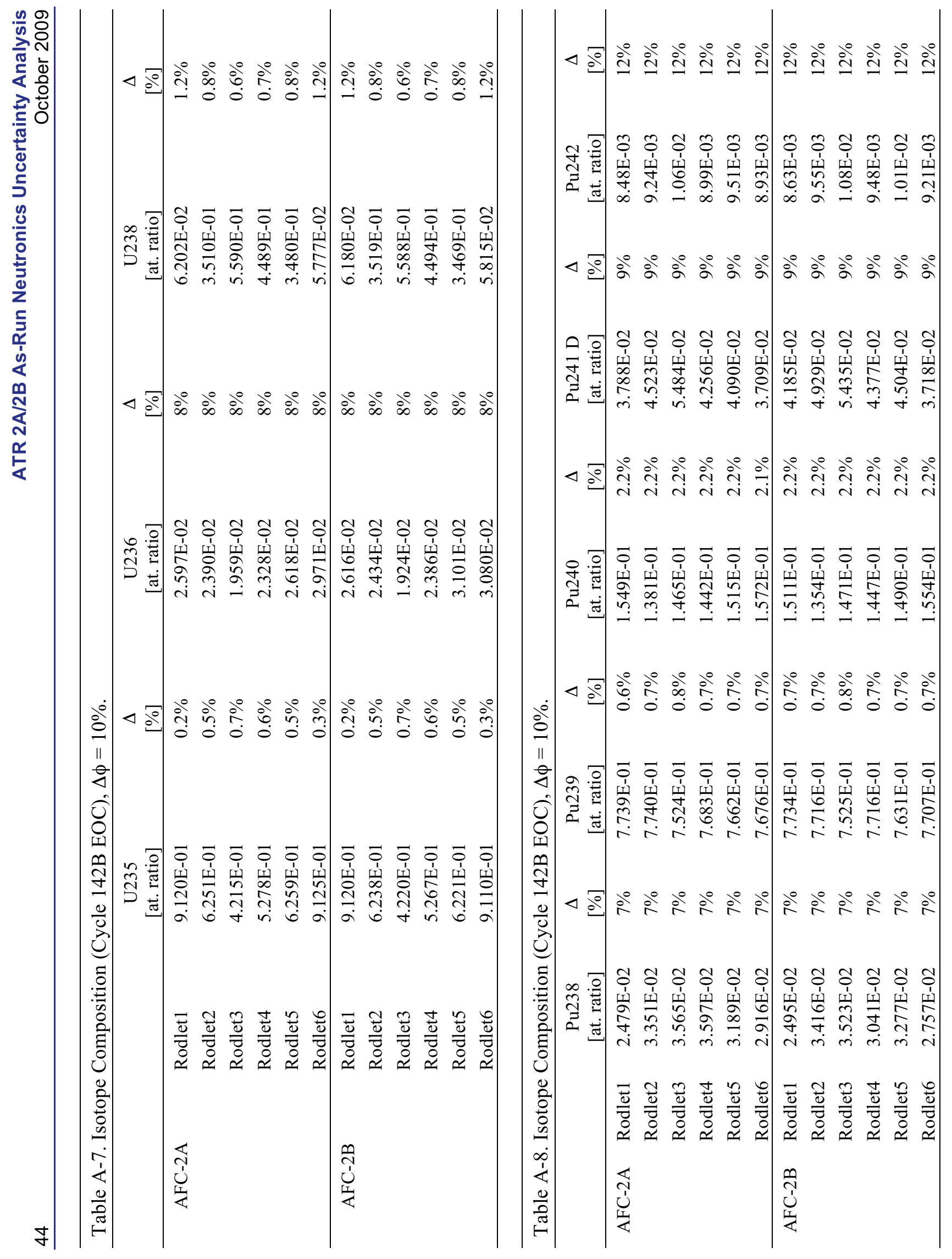




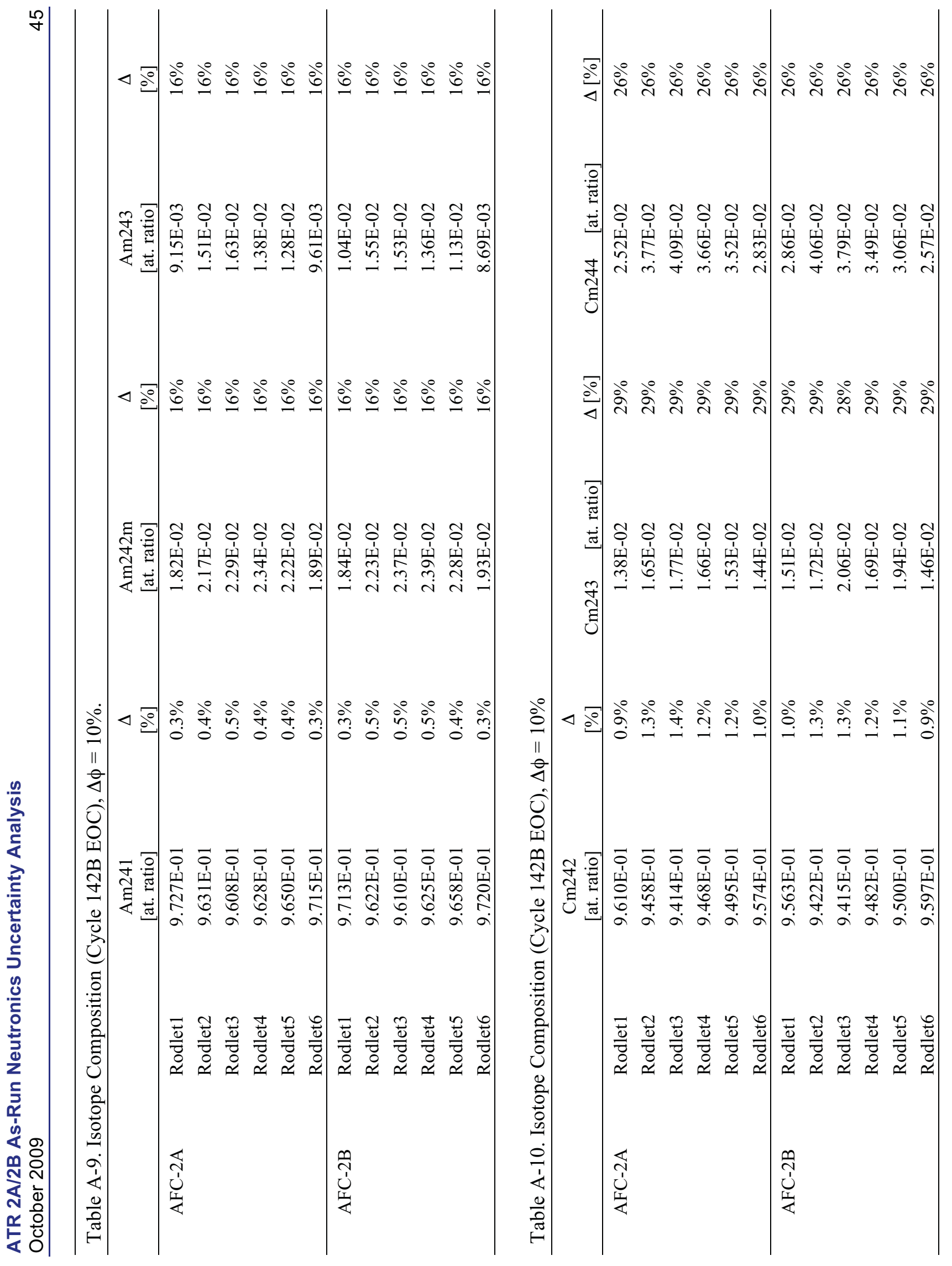


Table A-11. BURNUP (Cycle 142B EOC), $\Delta \phi=10 \%$.

\begin{tabular}{cccc}
\hline \multirow{7}{*}{ AFC-2A } & & $\begin{array}{c}\text { FIMA } \\
{[\Delta \text { HM/HMin }]}\end{array}$ & $\begin{array}{c}\Delta \\
{[\%]}\end{array}$ \\
& Rodlet1 & $4.621 \mathrm{E}-02$ & $19 \%$ \\
& Rodlet2 & $4.975 \mathrm{E}-02$ & $13 \%$ \\
& Rodlet3 & $4.642 \mathrm{E}-02$ & $12 \%$ \\
& Rodlet4 & $5.081 \mathrm{E}-02$ & $14 \%$ \\
& Rodlet5 & $5.197 \mathrm{E}-02$ & $14 \%$ \\
& Rodlet6 & $5.033 \mathrm{E}-02$ & $17 \%$ \\
\hline \multirow{2}{*}{ AFC-2B } & Rodlet1 & $4.697 \mathrm{E}-02$ & $18 \%$ \\
& Rodlet2 & $5.257 \mathrm{E}-02$ & $12 \%$ \\
& Rodlet3 & $4.640 \mathrm{E}-02$ & $12 \%$ \\
& Rodlet4 & $5.286 \mathrm{E}-02$ & $13 \%$ \\
& Rodlet5 & $5.425 \mathrm{E}-02$ & $14 \%$ \\
& Rodlet6 & $5.003 \mathrm{E}-02$ & $17 \%$ \\
\hline
\end{tabular}


Appendix B

\section{Cross Section and Fission Yield Error of RE Nuclide}




\section{B-1. Results of Isolated EFT Model with Boundary Neutron Source $(\Delta \phi=5-10 \%)$}
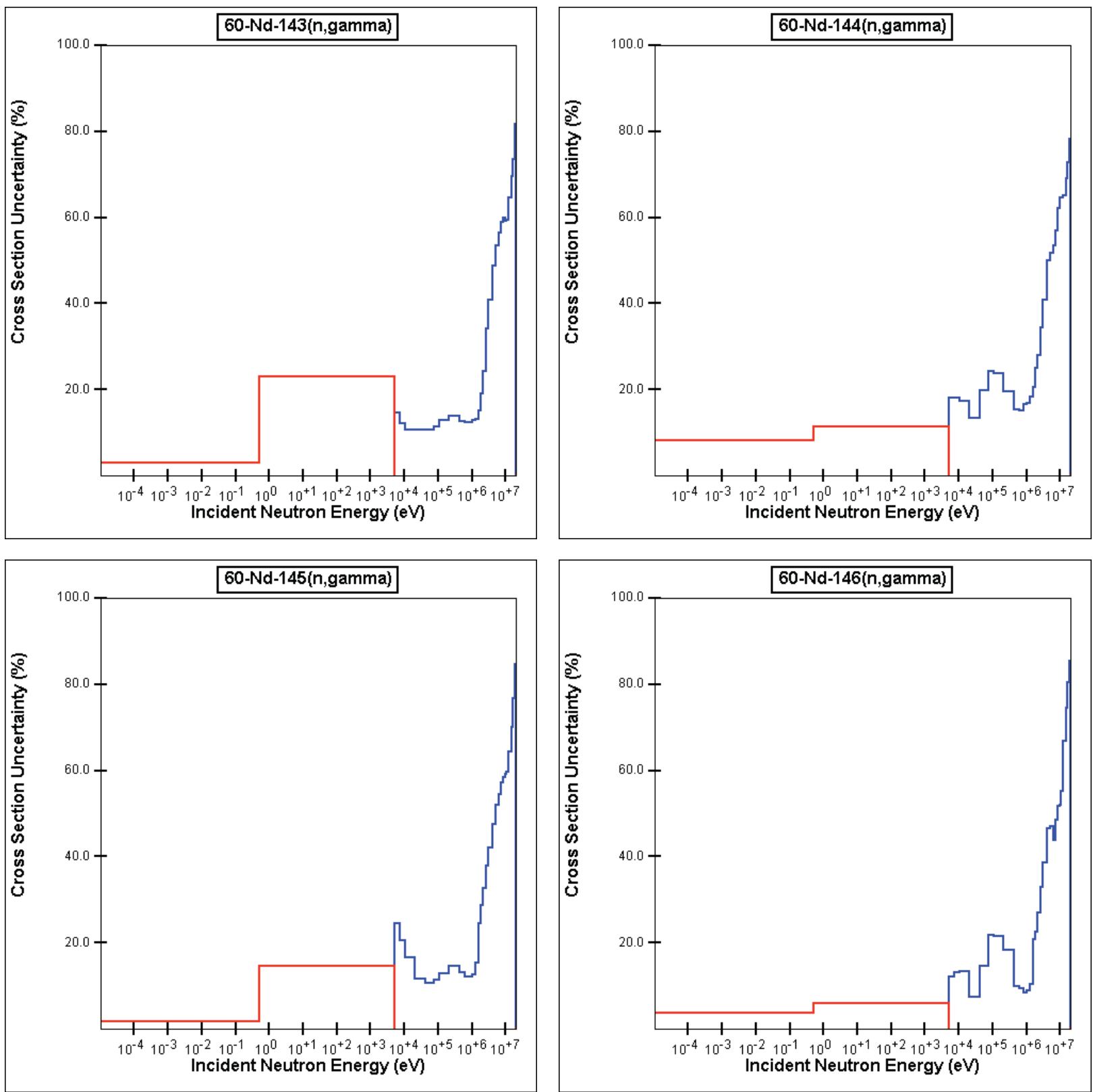

FigureB-1. Capture cross section error of $\mathrm{Nd}$ isotope (Data is derived from Low-Fidelity Covariance ${ }^{5}$ ). 

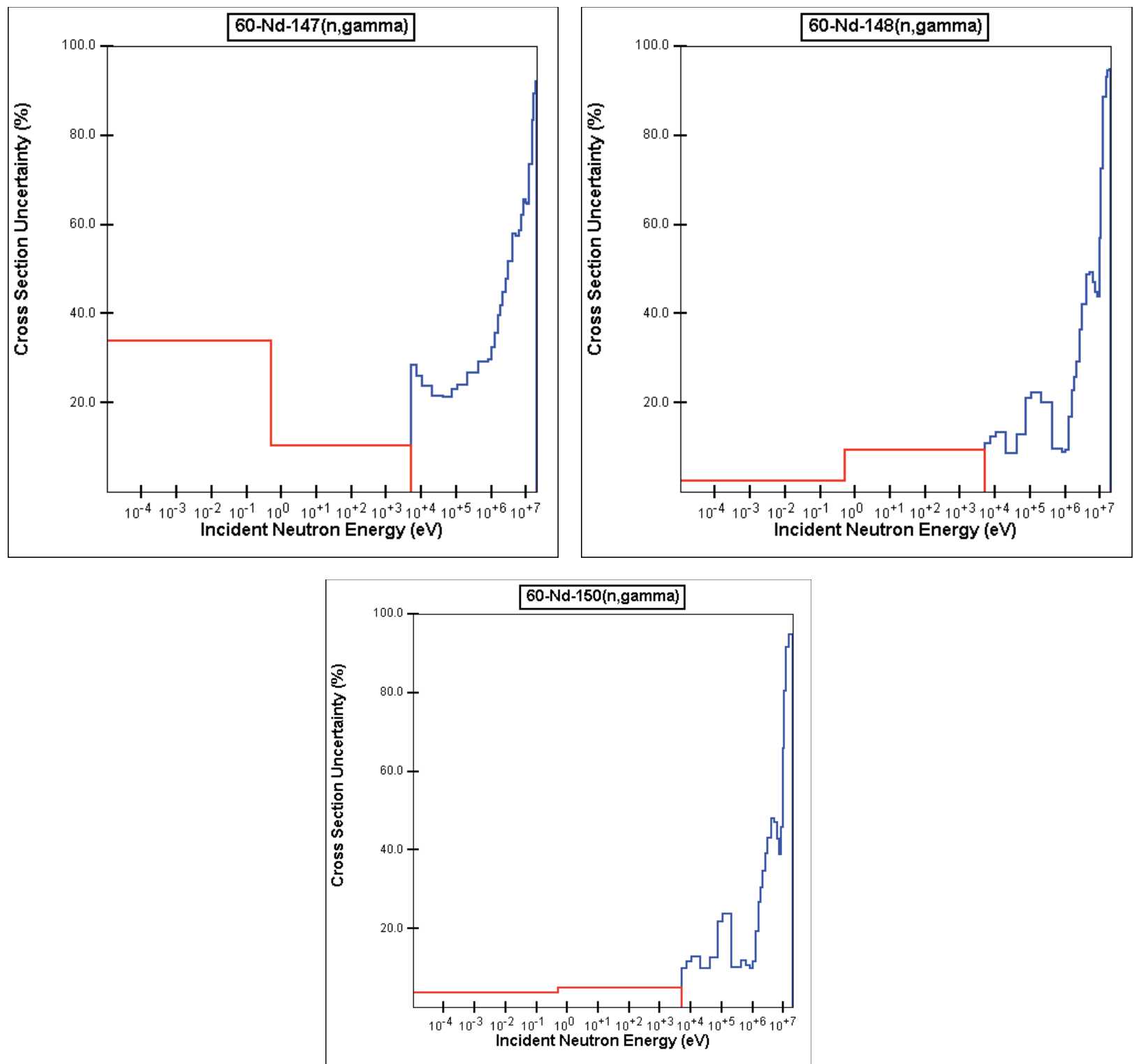

FigureB-2. Capture cross section error of $\mathrm{Nd}$ isotope (Data is derived from Low-Fidelity Covariance ${ }^{5}$ ). 

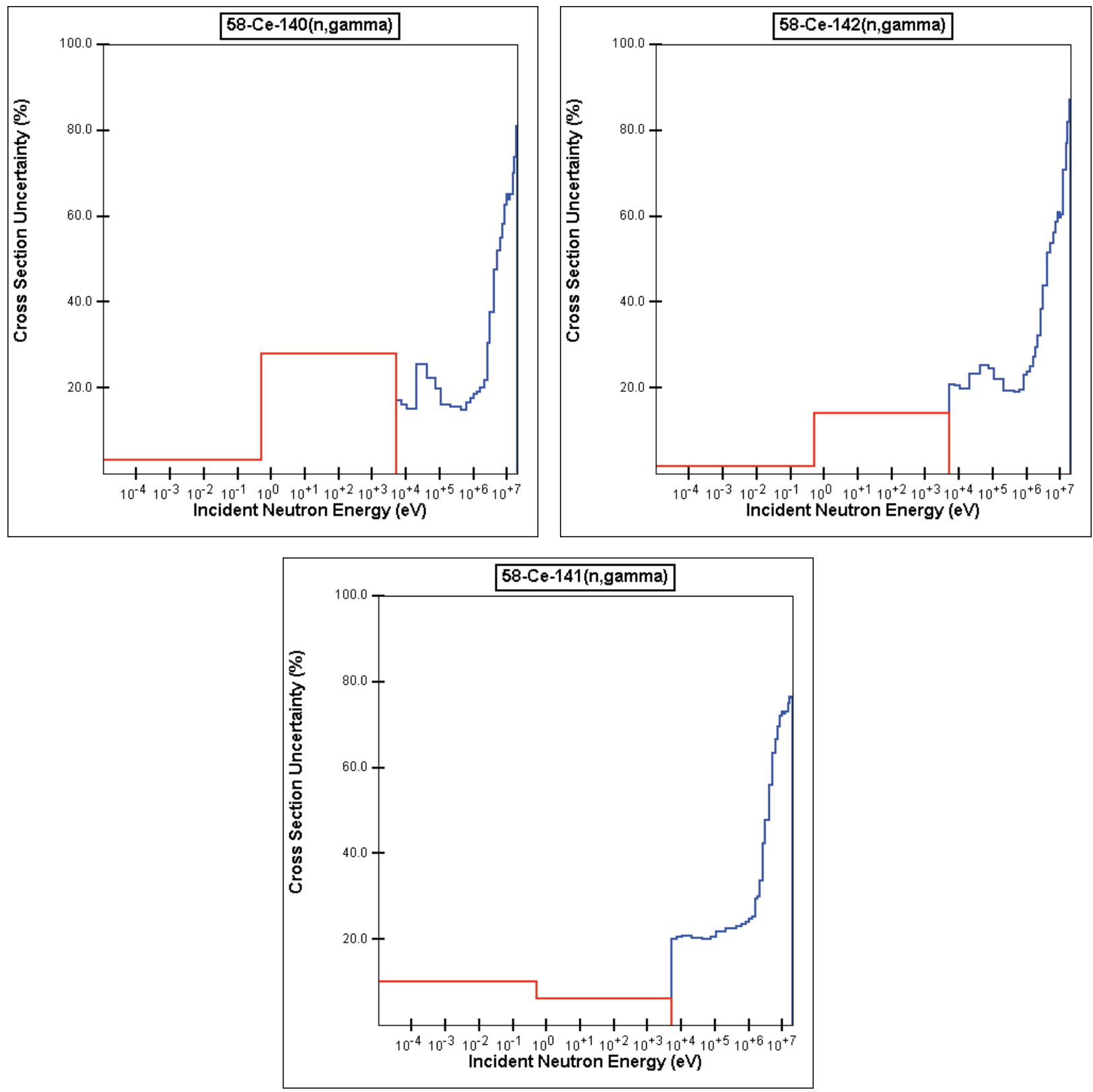

FigureB-3. Capture cross section error of Ce isotope (Data is derived from Low-Fidelity Covariance5). 

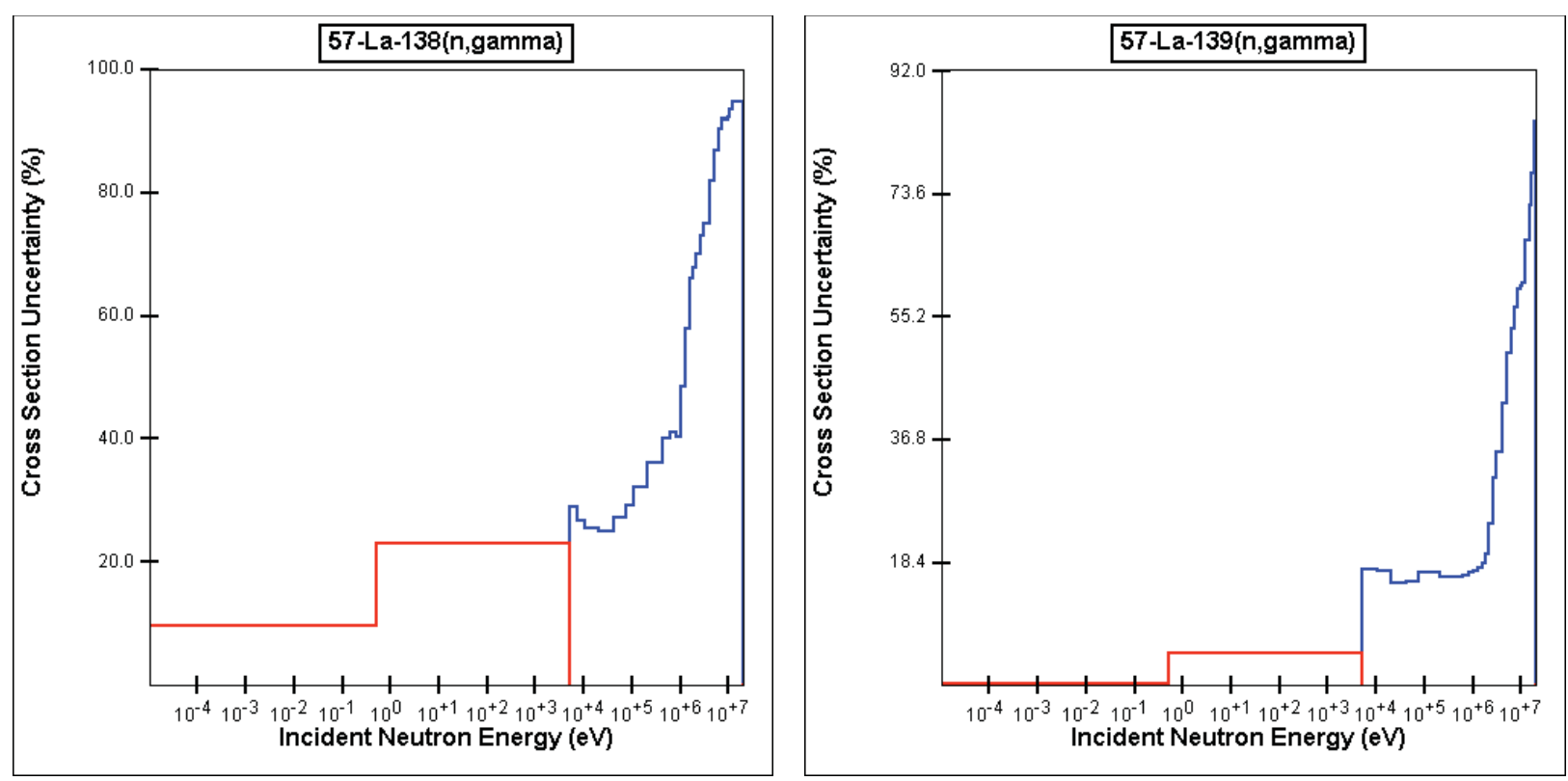

FigureB-4. Capture cross section error of La isotope (Data is derived from Low-Fidelity Covariance5).

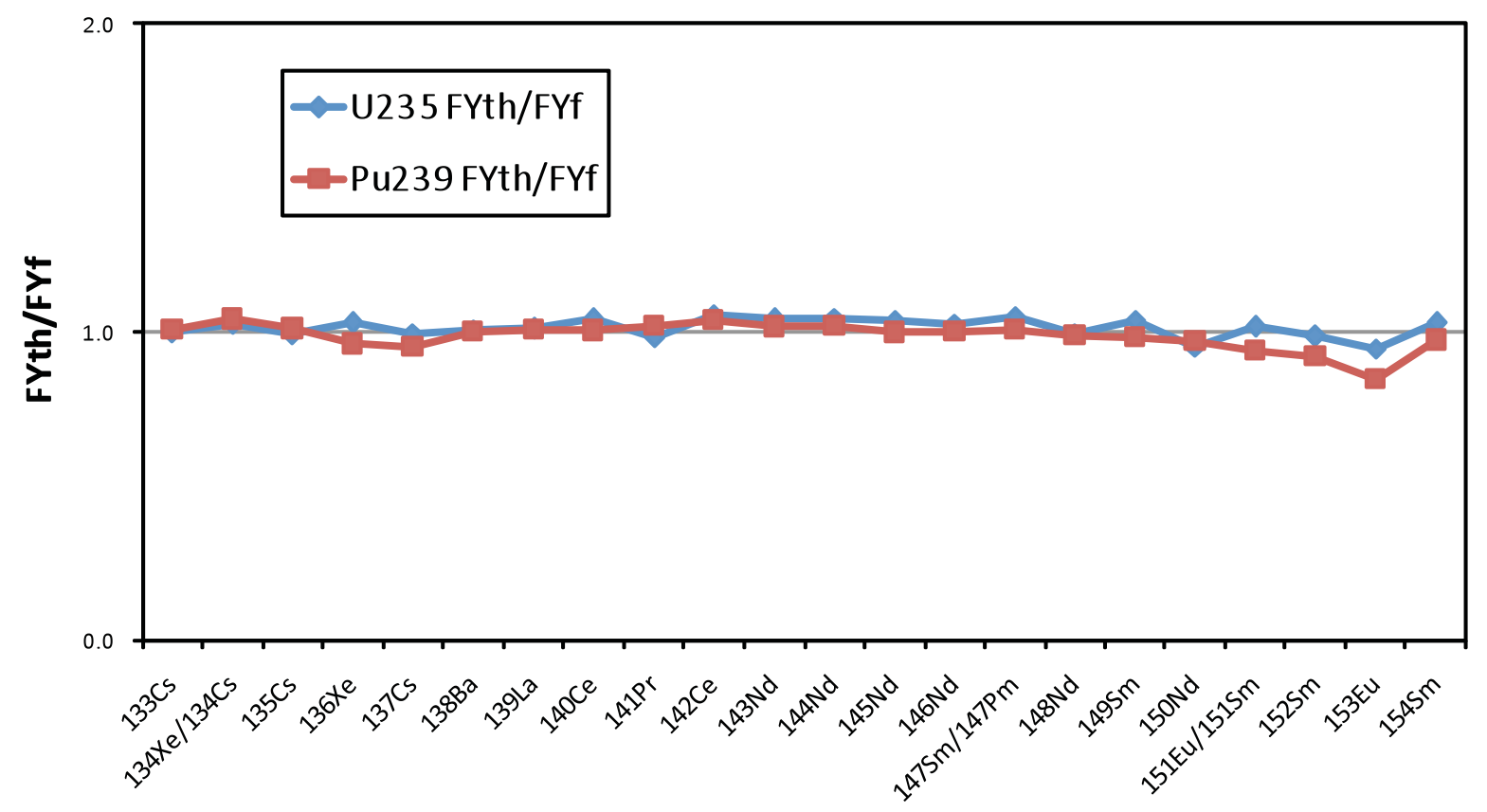

FigureB-5. Comparison of Cumulative Fission Yield between Thermal and Fast Injected Neutron Database (U-235 and Pu-239, ENDF/VII1). 


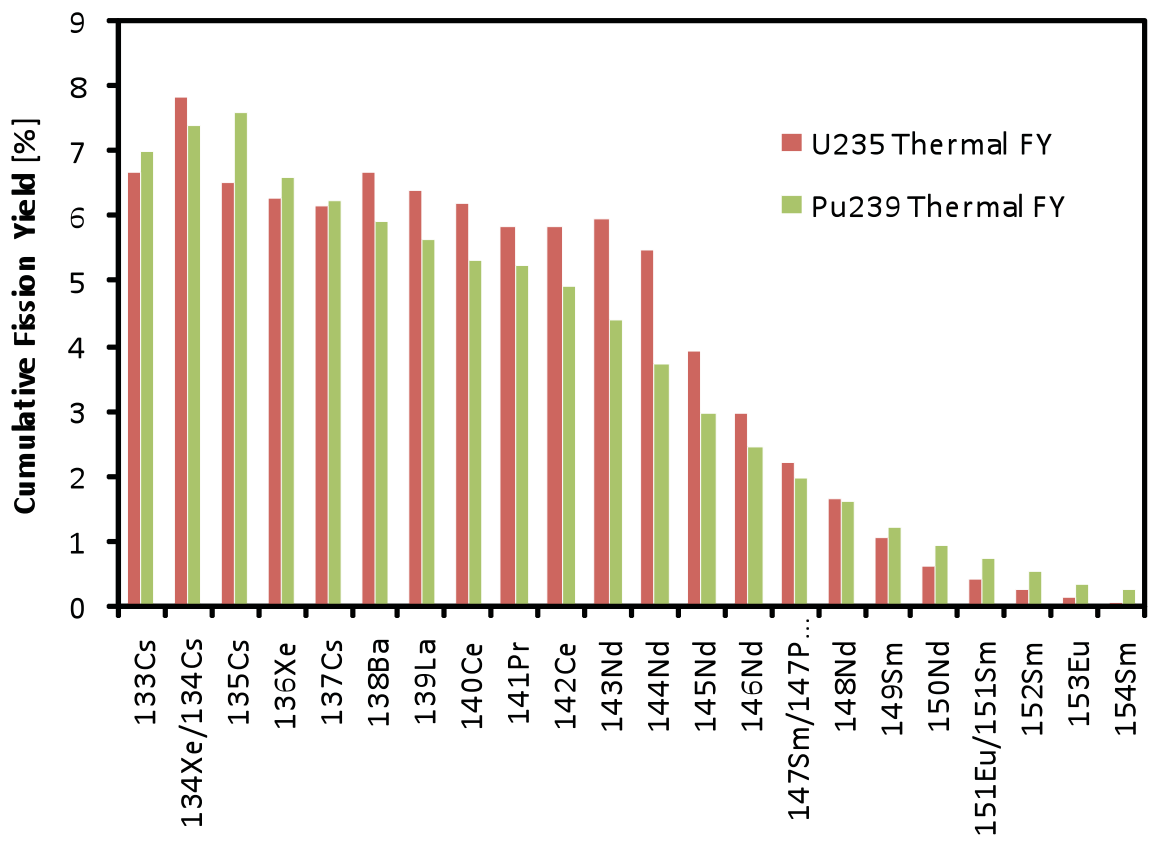

(a) Thermal Cumulative Fission Yield of REs

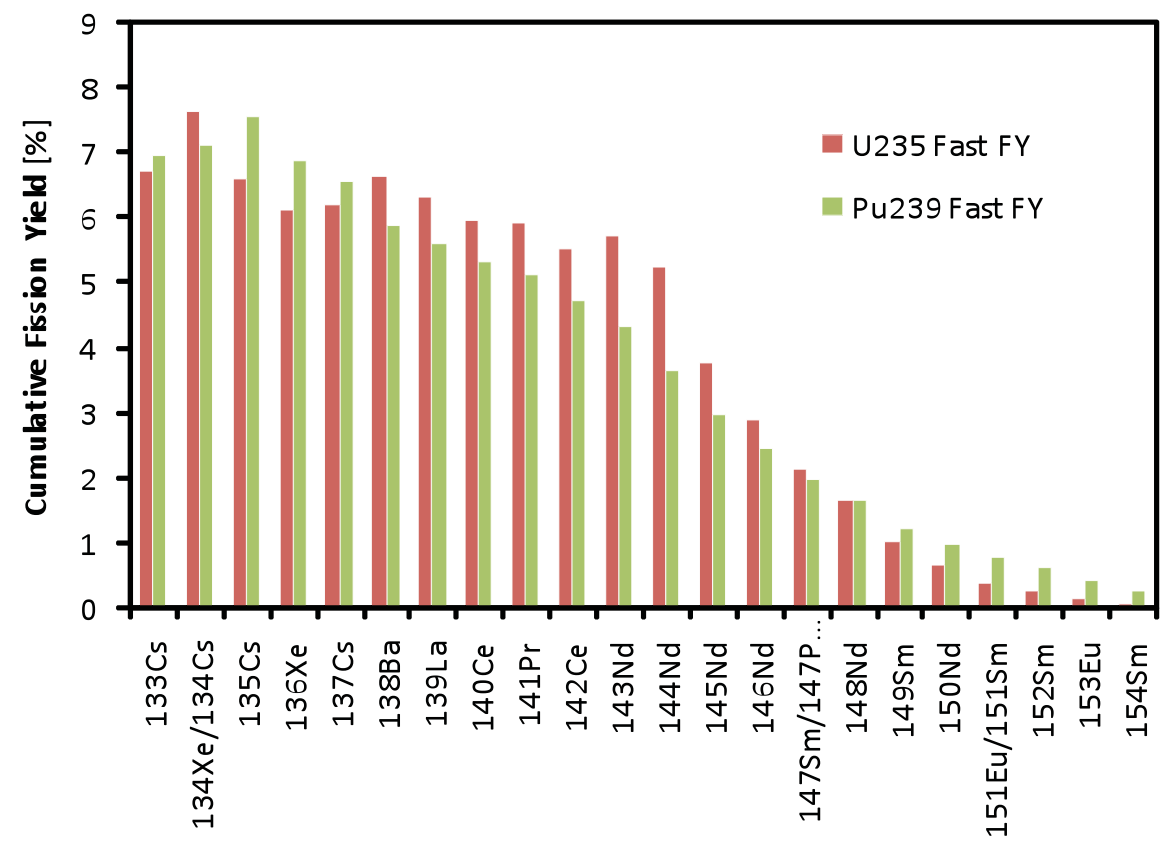

(b) Fast Cumulative Fission Yield REs

FigureB-6. Cumulative Fission Yield of U-235 and Pu-239 (ENDF/VII1). 